\title{
NEUTRINO SIGNATURES AND THE NEUTRINO-DRIVEN WIND IN BINARY NEUTRON STAR MERGERS
}

\author{
L. Dessart ${ }^{1,2}$, C. D. OtT ${ }^{1,3}$, A. Burrows ${ }^{2}$, S. Rosswog ${ }^{4}$, And E. Livne $^{5}$ \\ ${ }^{1}$ Department of Astronomy and Steward Observatory, The University of Arizona, Tucson, AZ 85721, USA; luc@as.arizona.edu \\ ${ }^{2}$ Department of Astrophysical Sciences, Princeton University, Princeton, NJ 08544, USA; burrows@ astro.princeton.edu \\ ${ }^{3}$ Theoretical Astrophysics, Mailcode 130-33, California Institute of Technology, Pasadena, CA 91125, USA; cott@tapir.caltech.edu \\ ${ }^{4}$ Jacobs University Bremen, Campus Ring 1, D-28759 Bremen, Germany; s.rosswog@ jacobs-university.de \\ ${ }^{5}$ Racah Institute of Physics, The Hebrew University, Jerusalem, Israel; livne@ phys.huji.ac.il \\ Received 2008 June 25; accepted 2008 September 4; published 2008 December 30
}

\begin{abstract}
We present VULCAN/2D multigroup flux-limited-diffusion radiation-hydrodynamics simulations of binary neutron star mergers, using the Shen equation of state, covering $\gtrsim 100 \mathrm{~ms}$, and starting from azimuthal-averaged twodimensional slices obtained from three-dimensional smooth-particle-hydrodynamics simulations of Rosswog \& Price for $1.4 M_{\odot}$ (baryonic) neutron stars with no initial spins, co-rotating spins, or counter-rotating spins. Snapshots are post-processed at $10 \mathrm{~ms}$ intervals with a multiangle neutrino-transport solver. We find polarenhanced neutrino luminosities, dominated by $\bar{v}_{e}$ and " $v_{\mu}$ " neutrinos at the peak, although $v_{e}$ emission may be stronger at late times. We obtain typical peak neutrino energies for $v_{e}, \bar{v}_{e}$, and " $v_{\mu}$ " of $\sim 12, \sim 16$, and $\sim 22 \mathrm{MeV}$, respectively. The supermassive neutron star (SMNS) formed from the merger has a cooling timescale of $\lesssim 1 \mathrm{~s}$. Charge-current neutrino reactions lead to the formation of a thermally driven bipolar wind with $\langle\dot{M}\rangle \sim$ $\tilde{10} 0^{-3} M_{\odot} \mathrm{s}^{-1}$ and baryon-loading in the polar regions, preventing any production of a $\gamma$-ray burst prior to black hole formation. The large budget of rotational free energy suggests that magneto-rotational effects could produce a much-greater polar mass loss. We estimate that $\lesssim 10^{-4} M_{\odot}$ of material with an electron fraction in the range $0.1-0.2$ becomes unbound during this SMNS phase as a result of neutrino heating. We present a new formalism to compute the $v_{i} \bar{v}_{i}$ annihilation rate based on moments of the neutrino-specific intensity computed with our multiangle solver. Cumulative annihilation rates, which decay as $\sim t^{-1.8}$, decrease over our $100 \mathrm{~ms}$ window from a few $\times 10^{50}$ to $\sim 10^{49} \mathrm{erg} \mathrm{s}^{-1}$, equivalent to a few $\times 10^{54}$ to $\sim 10^{53} \mathrm{e}^{-} \mathrm{e}^{+}$pairs per second.
\end{abstract}

Key words: gamma rays: bursts - hydrodynamics - neutrinos - stars: neutron - stars: rotation - supernovae: general

Online-only material: color figures

\section{INTRODUCTION}

Coalescing neutron stars are one of the primary progenitor candidates for short-duration (i.e., $\lesssim 2 \mathrm{~s}$ ) $\gamma$-ray bursts (GRBs; Paczynski 1986; Goodman 1986; Eichler et al. 1989; Narayan et al. 1992; Mochkovitch et al. 1993). In-spiral of the two neutron star components occurs due to energy and angular-momentum loss through gravitational radiation (Taylor \& Weisberg 1982; Weisberg \& Taylor 2005), which is emitted as the system evolves toward coalescence. Modern $\gamma$-ray and X-ray satellites have considerably improved our understanding of short-hard GRBs (Nakar 2007), and have more strongly associated them with binary neutron star (BNS) merger events (Lee \& RamirezRuiz 2007). Gravitational wave detectors such as the Laser Interferometer Gravitational-Wave Observatory (Abramovici et al. 1992) will most likely improve our understanding of these events in the coming decade.

The engine powering such short-hard GRBs and their associated high-Lorentz-factor ejecta is thought to be linked to mass accretion from a torus onto a central black hole, formed when the supermassive neutron star (SMNS) born from the coalescence eventually collapses. In recent years, models of GRB production from a high-mass, magnetar-like neutron star have also received some attention (Usov 1992; Usov 1994; Kluźniak \& Ruderman 1998; Rosswog et al. 2003; Dai et al. 2006; Metzger et al. 2007; Dessart et al. 2007). We will address the likelihood that the SMNS produces a GRB prior to black hole formation in Section 3.2. A fraction of the gravitational energy in the disk around this black hole is radiated as neutrinos, which power a disk wind. A fraction of these neutrinos and antineutrinos annihilate into $\mathrm{e}^{-} \mathrm{e}^{+}$. Magnetic processes may, through their role in angular-momentum transport, be decisive in setting the timescale between merger and collapse and, in addition, they may extract rotational energy from the central black hole (Blandford \& Znajek 1977). The ejecta may be confined by the magnetic field morphology or by the intense neutrino-driven baryon-loaded wind thought to accompany coalescence and black hole mass accretion (Levinson \& Eichler 2000; Rosswog \& Ramirez-Ruiz 2003; Aloy et al. 2005).

Numerical simulations of BNS mergers have a rich history, and have been considerably refined over the years, moving from two-dimensional to three-dimensional; from Newtonian to post-Newtonian, conformally flat, and finally to full general relativity (GR); from simple polytropic to more sophisticated equations of state (EOS); from the neglect of neutrinos to approximate neutrino-trapping schemes. BNS merger studies yield predictions for (1) their associated gravitational wave signals (Ruffert et al. 1996; Calder \& Wang 2002; Faber \& Rasio 2002; Shibata \& Uryū 2002; Shibata et al. 2003, 2005; Oechslin \& Janka 2007; Anderson et al. 2008b), (2) the production of short-hard GRBs (Ruffert et al. 1997; Ruffert \& Janka 1999; Rosswog \& Ramirez-Ruiz 2003; Rosswog et al. 2003; Rosswog 2005; Janka et al. 2006; Birkl et al. 2007), and (3) the pollution of the environment by r-process nuclei (Lattimer \& Schramm 1974, 1976; Symbalisty \& Schramm 1982; Eichler et al. 1989; 
Davies et al. 1994; Freiburghaus et al. 1999; Rosswog et al. 1999; Surman et al. 2008).

Different modeling ingredients and approaches have been employed. Three-dimensional Newtonian simulations without neutrino transport were performed by Oohara \& Nakamura (1989, 1990), Nakamura \& Oohara (1989, 1991), Zhuge et al. (1994, 1996), and Rosswog et al. (2000). Ruffert et al. (1996, 1997) included neutrinos using a grid-based code, and this was followed by Rosswog \& Davies (2002) and Rosswog \& Liebendörfer (2003) using a three-dimensional smooth-particlehydrodynamics (SPH) code. Recently, Setiawan et al. (2004, 2006) applied such a neutrino-leakage scheme in their study of torus-disk mass accretion around a black hole formed in a BNS merger, and addressed neutrino emission and annihilation. Magnetic fields in BNS merger evolution were introduced in three-dimensional Newtonian simulations by Price \& Rosswog (2006) and Rosswog \& Price (2007).

In parallel, there have been improvements to the Newtonian approach to incorporate the effects of GR (although largely neglecting microphysics), which become important as the two neutron stars come closer and eventually merge. Post-Newtonian simulations were performed by Oohara \& Nakamura (1992), Faber \& Rasio (2000, 2002), Faber et al. (2001), Ayal et al. (2001), and Calder \& Wang (2002). The conformally flat approximation was introduced in Wilson et al. (1996) and followed by Oechslin et al. (2002), Faber et al. (2004), Oechslin \& Janka (2006, 2007), and Oechslin et al. (2007). Full two-dimensional GR simulations, sometimes including magnetic fields, have been performed for SMNSs, possibly resulting from BNS mergers, by Baumgarte et al. (2000), Morrison et al. (2004), Duez et al. (2004, 2006), and Shibata et al. (2006), while full threedimensional GR simulations of the merger were carried out by Shibata \& Uryū (2002); Shibata et al. (2003, 2005); Shibata \& Uryu (2007), Anderson et al. (2008), and Baiotti et al. (2008).

All these simulations have been conducted at various levels of sophistication for the thermodynamic properties of matter, ranging from simplistic and not-so-physically-consistent polytropic EOSs to those employing a detailed microphysical representation of nuclear matter at an arbitrary temperature (Lattimer \& Swesty 1991; Shen et al. 1998a, 1998b). Investigations performed with such detailed EOSs have been conducted by, e.g., Ruffert et al. (1996, 1997), Rosswog et al. (1999), and Rosswog \& Davies (2002), and the dependency of BNS merger properties on the adopted EOS has been discussed by Oechslin \& Janka (2007) and Oechslin et al. (2007). Variations of a few times $10 \%$ in the maximum-allowed mass are seen, depending on the compressibility of nuclear matter, but recent observations may suggest that neutron stars with a gravitational mass in excess of $2 M_{\odot}$ do exist (Nice et al. 2005; Freire et al. 2007), supporting a stiff EOS for nuclear matter, such as the Shen EOS that we employ here. In Figure 1, we show, as a function of the central density, the baryonic and gravitational neutron star masses that we obtain for our implementation of the Shen EOS. The maximum occurs at $\sim 1.3 \times 10^{15} \mathrm{~g} \mathrm{~cm}^{-3}$, corresponding to a gravitational (baryonic) mass of $2.32 M_{\odot}\left(2.77 M_{\odot}\right){ }^{6}$ Moreover, in the context of differentially rotating (and possibly magnetized) SMNSs, the maximum mass that can be supported by a given EOS may be increased by up to $\sim 50 \%$ compared to the equivalent nonrotating object (Baumgarte et al. 2000; Morrison et al. 2004; Duez et al. 2004, 2006; Shibata et al. 2006; Sumiyoshi et al. 2007; Kiuchi \& Kotake 2008). Ultimately, understanding

\footnotetext{
6 Note that the values given in Dessart et al. (2008) are not exact.
}

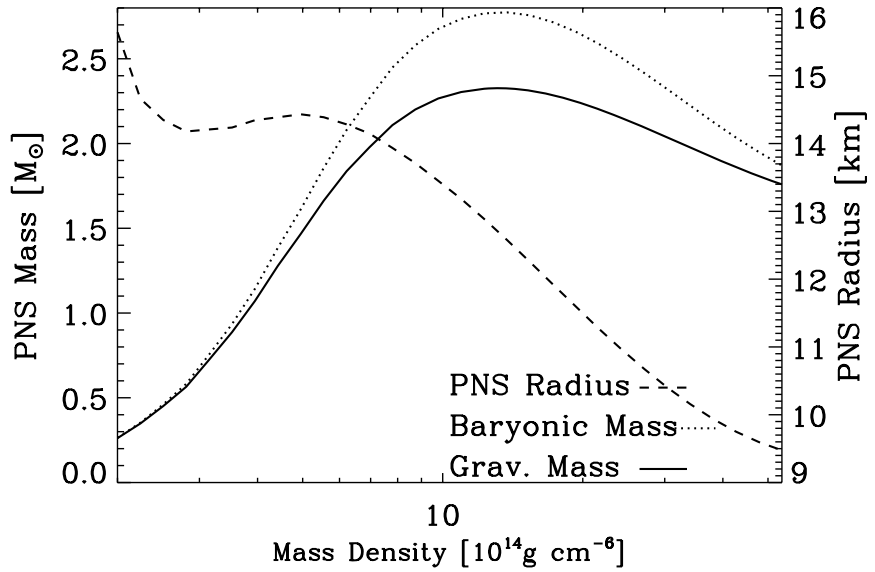

Figure 1. Gravitational (solid line) and baryonic (dotted line) masses, as well as the corresponding neutron star radius (dashed line), vs. central density derived for our Shen EOS and the Oppenheimer-Volkov equation (one form of the general-relativistic equation of hydrostatic equilibrium), and assuming a nonrotating neutron star characterized by a uniform electron fraction of 0.1 and a uniform temperature of $1 \mathrm{MeV}$. Here, the transition to a black hole will occur when the central density in the neutron star reaches $\sim 1.3 \times 10^{15} \mathrm{~g} \mathrm{~cm}^{-3}$, corresponding to a gravitational (baryonic) mass of $2.32 M_{\odot}\left(2.77 M_{\odot}\right)$ and a neutron star radius of $\sim 13 \mathrm{~km}$.

the mechanisms and timescales for angular momentum to be redistributed to lead to solid-body rotation is, therefore, important. One possible agency of redistribution is the magneto-rotational instability (Balbus \& Hawley 1991a; Akiyama et al. 2003; Pessah et al. 2006). The implications are nontrivial because the efficiency of angular-momentum transport determines in part whether a black hole forms promptly, or after a short or a long delay. It also determines how much mass can be accreted, i.e., whether there is $\sim 0.01$ or $\sim 0.1 M_{\odot}$ available in the torus surrounding the black hole after it has formed, and what the timescale is over which such accretion can take place to power relativistic ejecta. Although indirect, the relevance to short-hard GRBs and their properties is obvious, and perhaps central. In fact, this issue is also germane to the production of collapsars and long-duration GRBs (Dessart et al. 2008).

In this work, we perform two-dimensional multigroup, flux-limited-diffusion (MGFLD), radiation hydrodynamics of merged BNSs using the Shen EOS. The main goal of this work is to document in detail the neutrino signatures from such merger events. Our approach is to solve the neutrino transport problem self-consistently (although with a flux limiter and assuming diffusion), in combination with the dynamics of the system (but assuming axisymmetry), over $\gtrsim 100 \mathrm{~ms}$. At selected times, we post-process such models with the multiangle, $S_{n}$, neutrino transport algorithm of Livne et al. (2004), described recently in Ott et al. (2008). In particular, our investigation allows for the first time the computation of the neutrino-antineutrino annihilation rate from a solution of the transport equation, rather than using an approximate leakage scheme. Our investigation applies strictly to the neutron star phase of such BNS mergers.

This paper is organized as follows. In Section 2, we present the initial models we employ in our work, based on threedimensional SPH simulations of BNS mergers with different spin configurations (Section 2.1). In Section 2.2, we present the characteristics and procedures that we employ to evolve such initial conditions with the MGFLD radiation-hydrodynamics code VULCAN/2D. In Section 3, we present our results for the neutrino signatures (Section 3.1) and the dynamics of BNS mergers (Section 3.2). In Section 4, we address the neutrino- 
antineutrino annihilation rate in our three BNS merger models. We first present results employing the approach used so far (Ruffert et al. 1996, 1997; Rosswog \& Liebendörfer 2003; Setiawan et al. 2004, 2006; Birkl et al. 2007), based on a leakage scheme for the neutrino-flavor-dependent opacities and emissivities and a summation of all paired grid cells contributing at any given location. In Section 4.2, we then present a new formalism which uses moments of the neutrino-specific intensity computed with a multiangle, $S_{n}$, scheme. Finally, we present our conclusions in Section 5 and discuss the most striking implications for the powering of short-duration GRBs. For completeness, in the Appendix, we apply our formalism to the computation of the annihilation rate in the context of slowand fast-rotating single protoneutron stars (PNSs).

\section{MODEL DESCRIPTION}

The main goal of this work is to understand the longterm (over many rotation periods), post-coalescence, evolution of BNS mergers, with particular attention to neutrino signatures (flavor dependence, angular distribution, and radial distribution). Starting from azimuthal-averaged slices constructed from three-dimensional SPH simulations with the MAGMA code (Rosswog \& Price 2007) and taken a few milliseconds after coalescence, we perform two-dimensional (axisymmetric) MGFLD radiation-hydrodynamics simulations using the code VULCAN/2D (Livne et al. 2004; Dessart et al. 2006a; Burrows et al. 2007b; Ott et al. 2008). Below, we present the properties of the three BNS merger configurations from which we start (Section 2.1), and then describe our approach with VULCAN/2D in more detail (Section 2.2).

\subsection{Initial Conditions}

The MAGMA code (Rosswog \& Price 2007) is a state-of-theart SPH code that contains physics modules that are relevant to compact binary mergers and on top implements a slew of numerical improvements over most "standard" SPH schemes. A detailed code description can be found in Rosswog \& Price (2007), while results are presented in Price \& Rosswog (2006) and Rosswog (2007).

We briefly summarize the most important physics modules. For the thermodynamic properties of neutron star matter we use a temperature-dependent relativistic mean-field EOS (Shen et al. 1998a, 1998b). It can handle temperatures from 0 to $100 \mathrm{MeV}$, electron fractions from $Y_{\mathrm{e}}=0$ (pure neutron matter) up to 0.56 , and densities from about 10 to more than $10^{15} \mathrm{~g} \mathrm{~cm}^{-3}$. No attempt is made to include matter constituents that are more exotic than neutrons and protons at high densities.

The MAGMA code contains a detailed multiflavor neutrinoleakage scheme. An additional mesh is used to calculate the neutrino opacities that are needed for the neutrino emission rates at each particle position. The neutrino emission rates are used to account for the local cooling and the compositional changes due to weak interactions such as electron captures. A detailed description of the neutrino treatment can be found in Rosswog \& Liebendörfer (2003).

The self-gravity of the fluid is treated in a Newtonian fashion. Both the gravitational forces and the search for the particle neighbors are performed with a binary tree that is based on that described in Benz et al. (1990). These tasks are the computationally most expensive part of the simulations and in practice they completely dominate the CPU-time usage. Forces emerging from the emission of gravitational waves are treated in a simple approximation. For more details, we refer to the literature Rosswog et al. (2000); Rosswog \& Davies (2002).

In terms of numerical improvements over "standard" SPH techniques, the code contains the following:

1. To restrict shocks to a numerically resolvable width, artificial viscosity is used. The form of the artificial viscosity tensor is oriented at Riemann solvers (Monaghan 1997). The resulting equations are similar to those constructed for Riemann solutions of compressible fluid dynamics. In order to apply the artificial viscosity terms only where they are really needed, i.e., close to a shock, the numerical parameter that controls the strength of the dissipative terms is made time dependent, as suggested by Morris \& Monaghan (1997). An extra equation is solved for this parameter, which contains a source term that triggers on the shock and a term causing an exponential decay of the parameter in the absence of shocks. Tests can be found in Rosswog et al. (2000) and an illustration of the timedependent viscosity parameter in the context of Sod's shock-tube problem can be found in (Rosswog et al. 2008, their Figure 1).

2. A self-consistent treatment of extra terms in the hydrodynamics equations that arise from varying smoothing lengths (the so-called grad-h terms; Springel \& Hernquist 2002; Monaghan 2002; Price 2004; Rosswog \& Price 2007).

3. A consistent implementation of adaptive gravitational softening lengths as described in Price \& Monaghan (2007).

4. The option to evolve magnetic fields via so-called Euler potentials (Stern 1970) that guarantee that the constraint $\nabla \cdot B=0$ is fulfilled. The details can be found in Rosswog $\&$ Price (2007) and Rosswog \& Price (2008).

Our work starts from three-dimensional SPH simulations of BNS mergers in an isolated binary system, made up of two individual neutron stars, each with a baryonic mass of $1.4 M_{\odot}$ (equivalent to a gravitational mass of $\sim 1.3 M_{\odot}$ ), and separated by a distance of $48 \mathrm{~km}$. Their initial hydrostatic structure is computed by solving the Newtonian equations. The properties of nuclear matter (pressure, energy, entropy, etc.) are obtained by interpolation in the Shen EOS (Shen et al. 1998a, $1998 b)$, assuming zero temperature and $\beta$ equilibrium. A large number of SPH particles $\left(N \approx 10^{6}\right)$ are then mapped onto the resulting density profiles. These particles are further relaxed to find their true equilibrium state (see, e.g., Rosswog \& Price 2007).

Because the tidal synchronization time exceeds the gravitational decay time, BNSs cannot be tidally locked during their inspiral phase, and are thus expected to be close to irrotational (see, e.g., Bildsten \& Cutler 1992 and Kochanek 1992); however, past investigations of the coalescence of BNSs suggest that the intrinsic spin of each neutron star is an important parameter, as it determines, for example, the width of the baryon-poor funnel above the forming central object that is thought to play a decisive role in the launching of a GRB (see, e.g., Zhuge et al. 1996; Faber et al. 2001; Oechslin et al. 2007). Therefore, to encompass the range of possible outcomes, we simulate three initial configurations: (1) no spins (no intrinsic neutron star spin), (2) co-rotating spins (each neutron star has a period equal to that of the orbit, and rotates in the same direction), and (3) counter-rotating spins (same spin period as for co-rotating spins, but with rotation in a reverse direction to that of the orbit; the spins are counter-aligned). In the rest of this paper, we 
broadly refer to these models as the no-spin, the co-rotating spin, and the counter-rotating spin BNS models. The co-rotating and counter-rotating cases are included here to bracket the range of possibilities, but they represent unlikely extremes. We leave to future work the consideration of higher-mass BNS mergers (Rosswog \& Ramirez-Ruiz 2003) or asymmetric systems (Rosswog et al. 2000).

In practice, we post-process these three-dimensional SPH simulations by constructing azimuthal-averages, selecting a time after coalescence (when the two neutron stars come into contact) of $10.0 \mathrm{~ms}$ (no-spins), $12.4 \mathrm{~ms}$ (co-rotating spins), and $20.4 \mathrm{~ms}$ (counter-rotating spins), corresponding in each VULCAN/2D simulations to the time origin. At these times, these systems are evolving toward, but have not yet reached, complete axisymmetric configurations. This is a compromise made to model with VULCAN/2D the epoch of peak neutrino brightness. At these initial times, the amount of mass with a density greater than $10^{13} \mathrm{~g} \mathrm{~cm}^{-3}$ is $2.5 M_{\odot}$ in the no-spin BNS model, $2.31 M_{\odot}$ in the co-rotating spin model, and $2.43 M_{\odot}$ in the counter-rotating spin model. In the same model order, and at these times, the mass contained inside the $\sim 13 \mathrm{~km}$ radius of the highest mass neutron star allowed by the Shen EOS is only $1.11,1.26$, and $1.03 M_{\odot}$, respectively. The SPH simulations employed to generate these models are Newtonian, and, thus, one would expect more mass at small radii and systematically higher densities if allowance for GR effects were made, but the use of the highly incompressible Shen EOS, together with the significant amount of mass in quasi-Keplerian motion and on wide orbits, suggests that the systems should survive at least for some time before the general-relativistic gravitational instability occurs.

\subsection{VULCAN/2D}

Starting from fluid variables constructed with azimuthal averages of the three-dimensional SPH simulations described above, we follow the evolution of three BNS merger configurations with the Newtonian hydrodynamic code VULCAN/2D (Livne 1993), supplemented with an algorithm for neutrino transport as described in Livne et al. (2004) and Walder et al. (2005). The version of the code used here is the same as that discussed in Dessart et al. (2006a) and Burrows et al. (2006a), and uses the two-dimensional MGFLD method to handle neutrino transport (see Appendix A of Dessart et al. 2006a). Additional details on VULCAN/2D are provided in Burrows et al. (2007b). Magnetic fields are ignored in this study, as is the potential role of physical viscosity, i.e., there is no viscosity included besides one of an inherently numerical nature. The gravitational potential is assumed to be Newtonian and computed using a multipole solver (Dessart et al. 2006a). Our study presents a self-consistent treatment of neutrino emission, scattering, and absorption in a multispecies MGFLD context. Associated neutrino-matter coupling source terms are included in the momentum and energy conservation equations and their relevance to the merger evolution is, thus, computed explicitly for a typical duration of $\gtrsim 100 \mathrm{~ms}$. For these dynamical calculations, we include all neutrino processes described in Burrows et al. (2006b), but neglect the secondary processes of neutrino-electron scattering. The transport solution in the MGFLD scheme we employ is solved for the three neutrino species $v_{e}, \bar{v}_{e}$, and " $v_{\mu}$," (which groups together the $\mu$ and $\tau$ neutrinos) and at eight neutrino energies: $2.50,6.87,12.02,21.01,36.74,64.25$, 112.36 , and $196.48 \mathrm{MeV}$. The energy spacing is constant in the $\log$.
The choice of the spatial grid is determined primarily by the very aspherical density distribution of the merger and the very steep density drop-off at the neutron star surface in the polar direction. We present in Figures 2 and 3 (top row) the initial temperature (logarithmic scale and in $\mathrm{MeV}$ ), electron fraction, and the density distribution (white-line contours overplotted for every decade between $10^{7}$ and $10^{14} \mathrm{~g} \mathrm{~cm}^{-3}$ ) for each BNS merger configuration that we map onto the VULCAN/2D grid. Note that fast rotation in the inner region, although sub-Keplerian, displaces the density maximum by $8 \mathrm{~km}$ from the center and along the equator in the no-spin BNS model. These density peaks also correspond to extrema in the electron fraction of $\sim 0.1$ at this time. To setup the hybrid VULCAN/2D grid, we position the transition radius between the inner Cartesian and the outer spherical polar regions at $12 \mathrm{~km}$. The density at this location is at all times in excess of $10^{14} \mathrm{~g} \mathrm{~cm}^{-3}$, and thus-due to the stiffness of the Shen EOS in that regimeaway from the regions of large density gradient. The resolution in this inner region is typically $200 \times 200 \mathrm{~m}^{2}$, corresponding to $12 / 0.2=60$ zones in each (cylindrical) direction $r$ and $z$ from the center to the transition radius. Beyond the transition radius, we use a logarithmically increasing radial grid spacing with $\Delta r / r=1.85 \%$, using 301 zones to $3000 \mathrm{~km}$. The grid covers one hemisphere, from the rotation axis to the equator (both treated as reflecting boundaries), and the computation assumes axisymmetry, i.e., the azimuthal gradients are zero. We fill the grid outside the merger with material having a low density of $5 \times 10^{3} \mathrm{~g} \mathrm{~cm}^{-3}$ and a low temperature of $4 \times 10^{8} \mathrm{~K}$. Below $Y_{\mathrm{e}}=0.05$, we compute thermodynamic variables, as well as opacities/emissivities, by adopting an electron fraction of 0.05 . Given the smooth variation for the corresponding quantities (pressure, entropy, mean-free path, etc.) at this level of neutron richness, this approximation is expected to be quite good.

For example, the difference in any of these quantities between a neutron fraction $\left(1-Y_{\mathrm{e}}\right)$ of $95 \%$ and $100 \%$ (the latter would be the case for $Y_{\mathrm{e}}=0.0$ ) is approximately $5 \% \times$ (the neutron contribution - the proton contribution), corrected for the electron contribution difference, which is minuscule for cross sections. For the pressure, since the proton contribution at high densities is within $\sim 30 \%$ of the neutron contribution and at low densities is almost equal to the neutron contribution (ideal gas), ${ }^{7}$ the error is less than $\sim 1.5-2.0 \%$. For the scattering opacities, the universality of the neutral current makes this net error even smaller. For the electron-neutrino absorptive opacities, a high neutron fraction is accompanied by a low final-state proton blocking. For the anti-electron-neutrino absorption, a low proton fraction is accompanied by a high final-state neutron blocking factor. The net result is an absorption cross section at high densities that is only a few percent different for neutron fractions of $95 \%$ and $100 \%$.

After the MGFLD radiation-hydrodynamics simulations are completed, we post-process individual snapshots keeping the hydrodynamics frozen, and relaxing only the radiation quantities. This operation is performed for each model at $10 \mathrm{~ms}$ intervals over the whole evolution using the multiangle, $S_{n}$, radiation-transport module of Livne et al. (2004) and described more recently in Ott et al. (2008). We refer the reader to those papers for details on the method and the nomenclature. This $S_{n}$ variant yields the explicit angular dependence of the neutrinospecific intensity, and, thus, represents a more accurate modeling

\footnotetext{
7 Note that the electron pressure contribution below $Y_{e}=0.05$ and at high densities is fractionally less than $Y_{e}$, numerically due to the dominance of the strong nuclear force.
} 

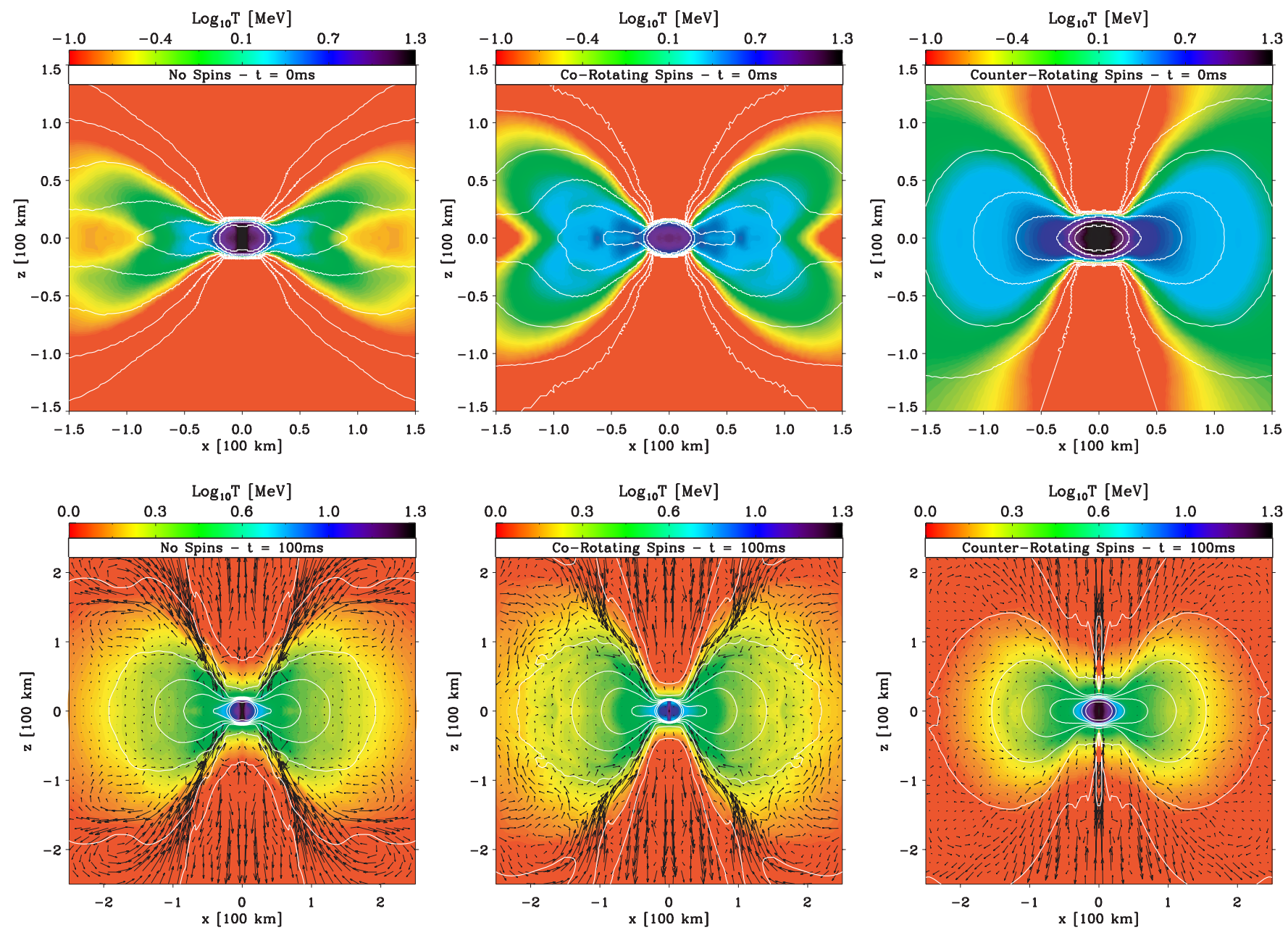

Figure 2. Colormap of the temperature (MeV; log scale) at 0 (top row; inner $150 \times 150 \mathrm{~km}^{2}$ region) and $100 \mathrm{~ms}$ (bottom row; inner $250 \times 250 \mathrm{~km}{ }^{2}$ region) after the start of the simulation. In the bottom panels, velocity vectors are added, with a saturation length at $15 \%$ of the width of the display and corresponding to a velocity of $30,000 \mathrm{~km} \mathrm{~s}^{-1}$. From left to right columns, we show the no-spin, co-rotating, and counter-rotating models. We also overplot white density contours for every decade, starting at a maximum of $10^{14} \mathrm{~g} \mathrm{~cm}^{-3}$. Note that the minimum of the color bar changes between the top and bottom rows.

(A color version of this figure is available in the online journal.)

of the strongly anisotropic neutrino luminosity in such highly aspherical systems (Ott et al. 2008). The $S_{n}$ calculation is done with the same number of energy groups (i.e., eight) and on the same spatial grid. In the $S_{n}$ algorithm, the transition at depth to MGFLD described in Ott et al. (2008) is natural here, since it occurs at $12 \mathrm{~km}$, and, thus, in regions where the densities are nuclear. However, the spatial resolution is not fully satisfactory at the neutron star surface and along the polar direction, a region where the density decreases by few orders of magnitude in just a few kilometers. Thus, this represents a challenge for radiation transport on our Eulerian grid. In the first instance, we relax the MGFLD results with $S_{n}$, adopting $8 \vartheta$-angles. In the $S_{n}$ approach, $n$ such $\vartheta$-angles translate into $n(n+2) / 2$ directions mapping the unit sphere uniformly. To obtain the steady-state angle-dependent radiation field, we apply the $S_{n}$ algorithm to initial conditions resulting from the converged MGFLD model. The solution is found by iterating on the radiation field at each time step until it does not change by more than one part in a thousand at any grid location. This newly found radiation field is then evolved in time through a number of consecutive time steps, until the solution has propagated through the entire grid. In the optically thick regions, the $S_{n}$ and the MGFLD solutions are inherently close, so convergence is reached after a few $\mathrm{ms}$, although about 10 iterations are required for convergence at each time step. In the optically thin regions, and in the regions intermediate between these two regimes, the convergence is obtained at each time step after 3-4 iterations, but the two solvers yield quite different results, so the $S_{n}$ run has to be evolved for at least $10 \mathrm{~ms}$, allowing the new multiangle radiation field to "propagate out" and fill the entire grid. Hence, after having reached $10 \mathrm{~ms}$, the $S_{n}$ run is stopped whenever the radiation field does not change by more than one part in a thousand at any location and from one time step to the next. Once converged, we perform an accuracy check by remapping the angle-dependent neutrino radiation field from 8 to $16 \vartheta$-angles, and then relaxing this higher angular-resolution simulation. Due to the increased computational costs, we use the high (16 $\vartheta$-angles) angular-resolution configuration only for snapshots at 10,60 , and $100 \mathrm{~ms}$, but for all three BNS merger configurations studied here. Importantly, the explicit angle dependence of the neutrino-radiation field allows us to compute its various moments, which we employ in a novel formulation of the neutrino-antineutrino annihilation rate (Section 4.2). Surprisingly, and as documented in Table 1 (see also Section 4.1), this sophisticated, i.e., multidimensional, multigroup, multiangle, approach that we follow yields results that agree favorably with those obtained using the leakage 

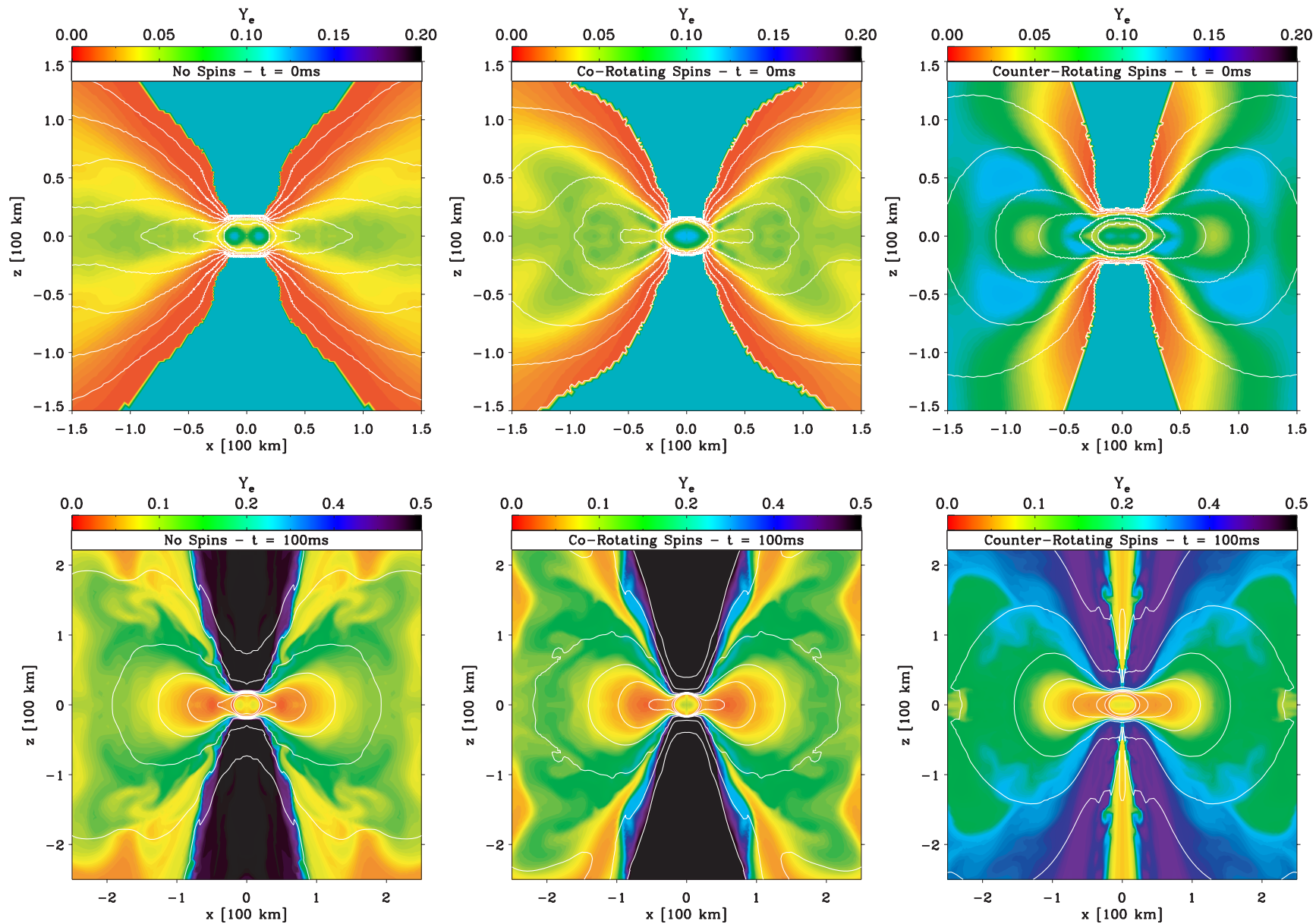

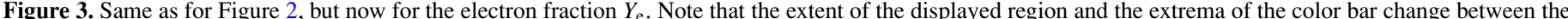

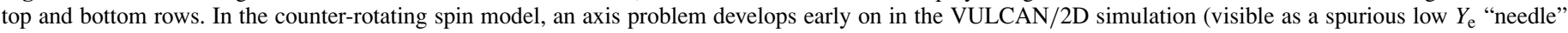
along the rotation axis), despite the high spatial resolution employed.

(A color version of this figure is available in the online journal.)

scheme of Ruffert et al. (1996, 1997), which by contrast is gray and makes rough estimates of directional diffusion times, scale heights, etc. (the expression of the diffusion time is itself, by essence, an approximation, based on random-walk arguments). Our computation of the angle dependence of the radiation field offers, however, a number of interesting new insights, which we explore in Section 4.

\section{BNS MERGER EVOLUTION AND NEUTRINO SIGNATURES}

In each model, the restart with VULCAN/2D is followed by a transient phase that lasts a few milliseconds and that is characterized by high-frequency oscillations of the highest density material, located within $10-20 \mathrm{~km}$ of the center. Given the good match between the Shen EOS used in the MAGMA code (Rosswog \& Price 2007) in this work (pressures differ by at most a few percent), we speculate that these oscillations are caused in part by the glitch introduced through the azimuthal averaging of the three-dimensional SPH simulation snaphsot. However, they may also reflect a fundamental dynamical property of this early phase. Similar oscillations are seen in three-dimensional simulations performed with MAGMA, and have also been reported in the literature, e.g., by Baiotti et al. (2008). The associated shocks generate thermal energy (note that the thermal part of the pressure is subdominant at nuclear densities), but this thermal component is negligible compared, for example, with what is needed to power the neutrino luminosities that we see. Any initial mismatch is thus merely a small transient which has a negligible impact on the long-term evolution of the BNS mergers that we study in this work.

The evolxsutions of the BNS merger models with initially no spins, co-rotating spins, and counter-rotating spins are qualitatively similar. As discussed above, at the start of the VULCAN simulations, the matter distribution is far from stationary, with a significant amount of material at subnuclear densities and at large distances from the center. Throughout the $\sim 100 \mathrm{~ms}$ of evolution that we follow, matter in the inner few hundred kilometers settles in and comes to rest at the neutron star surface, while material beyond a few hundred kilometers and located at low, near-equatorial latitudes migrates outward due to the large thermal pressure gradient and the strong centrifugal effects. This expansion also takes place in the vertical direction, leading to the formation of a low-density cocoon surrounding an inner and denser disk. In the intermediate region, i.e., at radii of 50-100 km and along the equatorial direction, material evolves toward quasi-Keplerian motion, with little inward or outward migration. In Figures 2 and 3, we show the $T-Y_{\mathrm{e}}$ distributions at the start of the VULCAN/2D simulations (top row), and $100 \mathrm{~ms}$ later (bottom row), together with isodensity contours (shown in white) for every decade starting at a maximum value of $10^{14} \mathrm{~g} \mathrm{~cm}^{-3}$. 
Table 1

$v_{i} \bar{v}_{i}$ Annihilation Rates Using the Leakage and the $S_{n}$ Schemes

\begin{tabular}{|c|c|c|c|c|c|c|}
\hline \multirow{2}{*}{$\begin{array}{l}\text { Time } \\
(\mathrm{ms})\end{array}$} & \multicolumn{2}{|c|}{ No Spins } & \multicolumn{2}{|c|}{ Co-Rotating Spins } & \multicolumn{2}{|c|}{ Counter-Rotating Spins } \\
\hline & \multicolumn{2}{|c|}{$\left(\mathrm{B} \mathrm{s}^{-1}\right)$} & \multicolumn{2}{|c|}{$\left(\mathrm{B} \mathrm{s}^{-1}\right)$} & \multicolumn{2}{|c|}{$\left(\mathrm{B} \mathrm{s}^{-1}\right)$} \\
\hline & \multicolumn{6}{|c|}{ Leakage Scheme } \\
\hline 10 & $1.56(-1)$ & $3.28(-5)$ & $2.05(-1)$ & $1.01(-4)$ & $2.18(-1)$ & $1.40(-4)$ \\
\hline 20 & $9.28(-2)$ & $5.99(-6)$ & $4.79(-2)$ & $2.38(-6)$ & $1.05(-1)$ & $1.01(-5)$ \\
\hline 30 & $3.80(-2)$ & $1.15(-6)$ & $3.70(-2)$ & $1.43(-6)$ & $5.95(-2)$ & $3.35(-6)$ \\
\hline 40 & $3.11(-2)$ & $8.54(-7)$ & $4.67(-2)$ & $1.08(-6)$ & $3.65(-2)$ & $1.36(-6)$ \\
\hline 50 & $2.82(-2)$ & $6.23(-7)$ & $5.67(-2)$ & $1.35(-6)$ & $2.16(-2)$ & $5.81(-7)$ \\
\hline 60 & $1.78(-2)$ & $2.57(-7)$ & $4.43(-2)$ & $8.74(-7)$ & $1.50(-2)$ & $3.00(-7)$ \\
\hline 70 & $1.32(-2)$ & $1.63(-7)$ & $2.69(-2)$ & $3.78(-7)$ & $1.18(-2)$ & $1.79(-7)$ \\
\hline 80 & $1.22(-2)$ & $1.67(-7)$ & $3.55(-2)$ & $8.16(-7)$ & $1.01(-2)$ & $1.21(-7)$ \\
\hline 90 & $1.47(-2)$ & $2.94(-7)$ & $2.96(-2)$ & $6.64(-7)$ & $7.64(-3)$ & $7.64(-8)$ \\
\hline 100 & $1.60(-2)$ & $3.73(-7)$ & $2.54(-2)$ & $5.41(-7)$ & $6.48(-3)$ & $5.47(-7)$ \\
\hline \multicolumn{7}{|c|}{$S_{n}$ Scheme } \\
\hline 10 & $1.81(-1)$ & $1.44(-2)$ & $5.97(-1)$ & $9.19(-3)$ & $3.63(-1)$ & $4.76(-2)$ \\
\hline 20 & $6.82(-2)$ & $6.41(-3)$ & $9.22(-2)$ & $1.15(-3)$ & $8.92(-2)$ & $1.70(-2)$ \\
\hline 30 & $3.95(-2)$ & $3.96(-3)$ & $4.59(-2)$ & $4.59(-4)$ & $4.08(-2)$ & $1.01(-2)$ \\
\hline 40 & $2.71(-2)$ & $2.58(-3)$ & $4.13(-2)$ & $2.82(-4)$ & $2.77(-2)$ & $7.83(-3)$ \\
\hline 50 & $2.18(-2)$ & $1.78(-3)$ & $4.19(-2)$ & $1.89(-4)$ & $1.92(-2)$ & $5.84(-3)$ \\
\hline 60 & $1.82(-2)$ & $1.39(-3)$ & $3.59(-2)$ & $1.34(-4)$ & $1.28(-2)$ & $4.27(-3)$ \\
\hline 70 & $1.47(-2)$ & $1.02(-3)$ & $2.31(-2)$ & $8.70(-5)$ & $1.04(-2)$ & $3.76(-3)$ \\
\hline 80 & $1.11(-2)$ & $6.94(-4)$ & $2.30(-2)$ & $6.22(-5)$ & $8.49(-3)$ & $3.28(-3)$ \\
\hline 90 & $1.01(-2)$ & $5.25(-4)$ & $1.84(-2)$ & $4.28(-5)$ & $7.13(-3)$ & $2.83(-3)$ \\
\hline 100 & $9.67(-3)$ & $3.93(-4)$ & $1.59(-2)$ & $3.02(-5)$ & $6.21(-3)$ & $2.46(-3)$ \\
\hline
\end{tabular}

Notes. Upper half: listing of the volume-integrated rates for the $v_{e} \bar{v}_{e}$ and " $v_{\mu} \bar{v}_{\mu}$ " annihilation rates using the approach of Ruffert et al. $(1996,1997)$, as summarized in Section 4.1. The time for each calculation refers to the time since the start of the VULCAN/2D simulation. Numbers in parenthesis correspond to powers of 10 . The strong decrease of the energy-deposition rates reflects the fading of the neutrino luminosity due to the cooling of the SMNS. A density cut above $10^{11} \mathrm{~g} \mathrm{~cm}^{-3}$ is applied for both emission and deposition sites. Lower half: same as the upper half, but this time using the $8 \vartheta$-angle $S_{n}$ calculations performed through a post-processing of the MGFLD radiation-hydrodynamics snapshots computed with VULCAN/2D. The same hydrodynamics background ( $\rho, T, Y_{\mathrm{e}}$ distribution) is used for the leakage and the $S_{n}$-schemes.

At $100 \mathrm{~ms}$ after the start of the VULCAN/2D simulations, each merger has reached a quasi-steady equilibrium configuration, characterized by a large equator to pole radius ratio of $5 / 1$, visible from the latitudinal variation of the extent of the $10^{10} \mathrm{~g} \mathrm{~cm}^{-3}$ density contour (the bottom row panels in Figure 2 and 3) or from radial slices of the density in the polar and equatorial directions (Figure 4). This is a general result for fast-rotating neutron stars (or other degenerate objects like white dwarfs) which has been documented in various contexts in the past (Ostriker \& Mark 1968; Hachisu 1986; Liu \& Lindblom 2001; Yoon \& Langer 2005; Rosswog \& Davies 2002; Kiuchi \& Kotake 2008). At the start of the VULCAN simulations, high-density material was located in a thin $(\lesssim 20 \mathrm{~km})$ structure extending $\lesssim 300 \mathrm{~km}$ in the equatorial direction. After $100 \mathrm{~ms}, 90 \%$ of the total mass is contained within $30 \mathrm{~km}$ of the center (corresponding to $\sim 2.5 M_{\odot}$ ) and at densities greater than $10^{13} \mathrm{~g} \mathrm{~cm}^{-3}$. At the same time, the remaining $\sim 10 \%$ (corresponding to $\sim 0.2 M_{\odot}$ ) is located in a quasi-Keplerian disk with an outer edge at $\lesssim 100 \mathrm{~km}$ (Figures 4 and 5; see also Section 3.1). This is also illustrated in Figure 6 for the no-spin model and at $60 \mathrm{~ms}$ after the start of the VULCAN/2D simulation, a figure in which we show the interior baryonic mass as a function of spherical radius, and how it compares with various multiples of the Schwarzschild radius.

\subsection{Neutrino Signatures}

Neutrino processes of emission and absorption/scattering in BNS merger simulations have been treated using leakage schemes by Ruffert et al. (1996, 1997), Rosswog \& Liebendörfer (2003), Setiawan et al. (2004, 2006), and Birkl et al. (2007). In this approach, the difficult solution of the multiangle, multigroup Boltzmann transport equation is avoided by introducing a neutrino-loss timescale, for both energy and number, and applied to optically thick and optically thin conditions. In addition, an estimated rate of electron-type neutrino loss allows the electron fraction to be updated. Overall, benchmarking of these leakage schemes using more sophisticated one-dimensional core-collapse simulations that solve the Boltzmann equation suggests that the resulting neutrino luminosities are within a factor of a few (at most) of what would be obtained using a more accurate treatment.

The work presented here offers a direct test of this. Moreover, because the neutrino source terms are included in the momentum and energy equations solved by VULCAN/2D, we can model the birth and subsequent evolution of the resulting neutrinodriven wind. Our simulations being two-dimensional and less CPU intensive than three-dimensional SPH simulations can also be extended to $\gtrsim 100 \mathrm{~ms}$, and, thus, we can investigate the longterm evolution of the merger. In particular, we directly address the evolution of the neutrino luminosity and confront this with the results using the expedient of a steady state often employed in work that is also limited to a short time span of $10-20 \mathrm{~ms}$ after the merger.

In the leakage schemes of Ruffert et al. $(1996,1997)$ and Rosswog \& Liebendörfer (2003), the neutrino emission processes treated are electron and positron capture on protons 

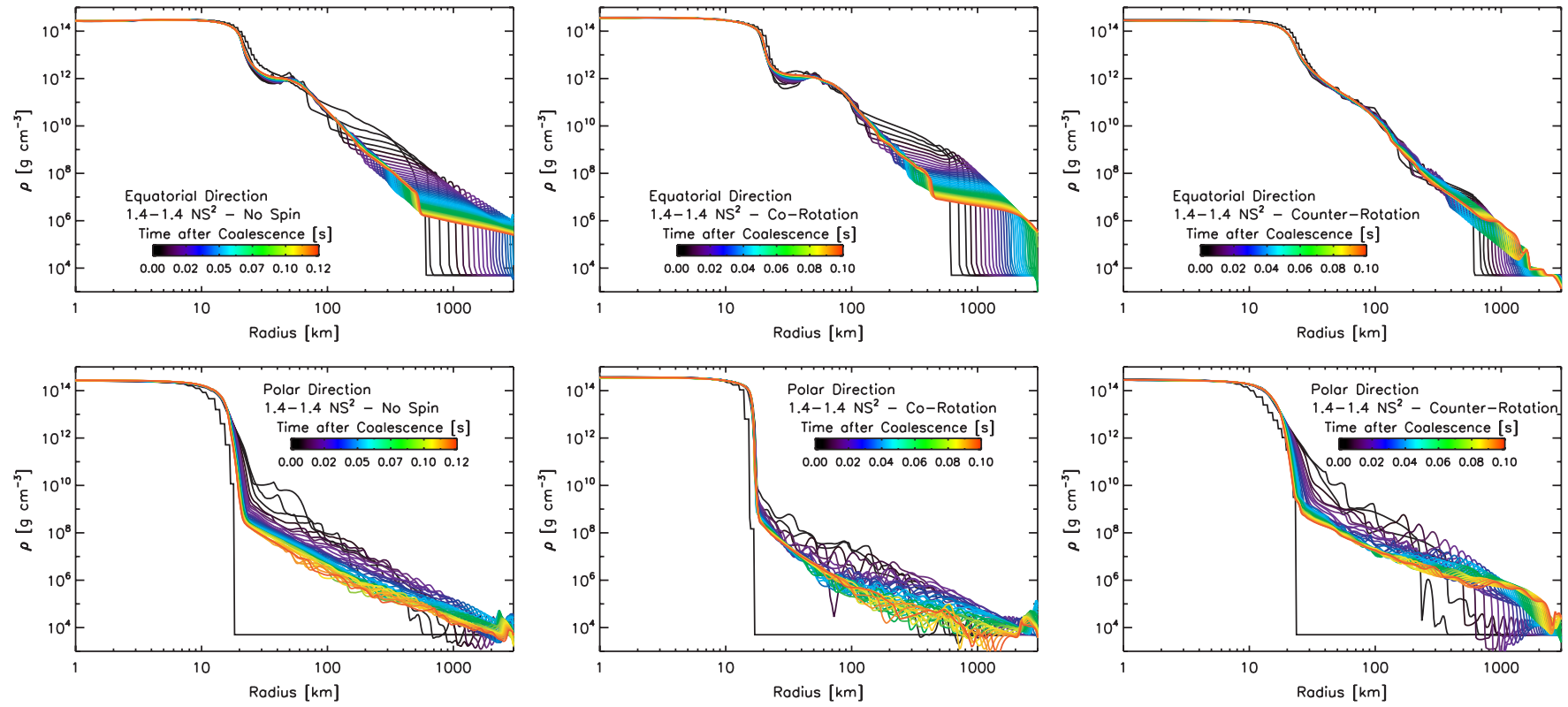

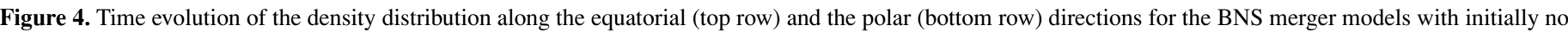
spins (left), co-rotating spins (middle), and counter-rotating spins (right).

(A color version of this figure is available in the online journal.)
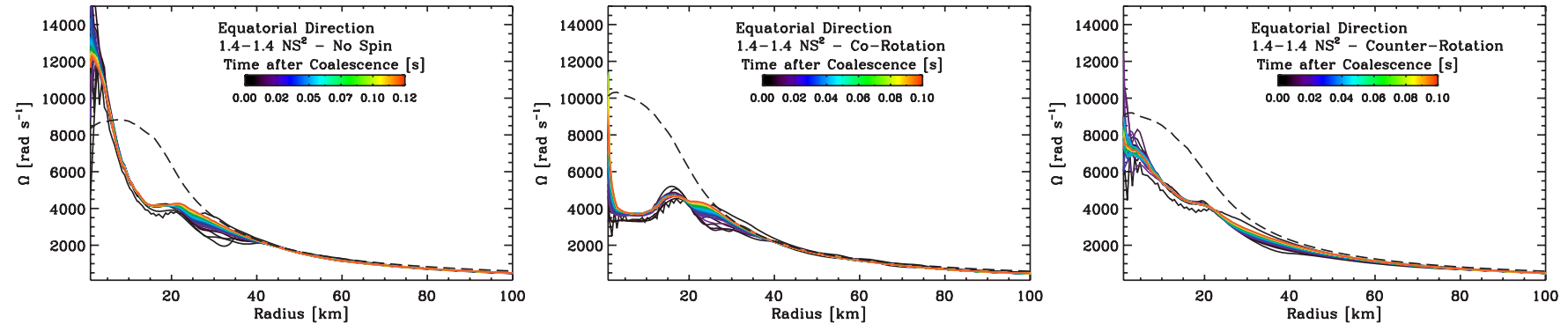

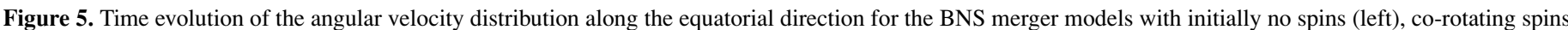

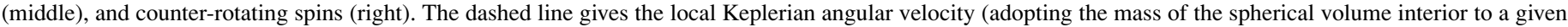

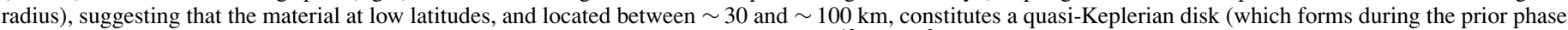

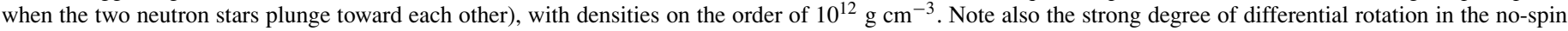
and counter-rotating spin cases, which is preserved throughout the VULCAN/2D simulation.

(A color version of this figure is available in the online journal.)

(yielding $v_{e}$ ) and neutrons (yielding $\bar{v}_{e}$ ), respectively, and electron-positron pair annihilation and plasmon decay (each yielding electron-, $\mu$-, and $\tau$-type neutrinos). For electron-type neutrinos, the dominant emission processes are the chargedcurrent $\beta$-processes. For neutrino opacity, Ruffert et al. (1996, 1997) include neutrino scattering on nucleons. Rosswog \& Liebendörfer (2003) also treat electron-type neutrino absorption on nucleons.

The neutrino emission, absorption, and scattering processes used in VULCAN/2D simulations are those summarized in Burrows et al. (2006b), and include all the above, plus electron neutrino absorption on nuclei and nucleon-nucleon bremsstrahlung. The latter is the dominant emission process of $v_{\mu}$ and $v_{\tau}$ neutrinos in PNSs (Thompson et al. 2000). In VULCAN/2D, the $v_{\mu}$ and $v_{\tau}$ neutrinos are grouped together and referred to as " $v_{\mu}$ " neutrinos. We present in Figure 7 the evolution of the neutrino luminosity for the BNS merger models with initially no spins (top), co-rotating spins (center), and counter-rotating spins (bottom) over a typical timespan of $\gtrsim 100 \mathrm{~ms}$, and for an adopted radius of $200 \mathrm{~km}$ along the equatorial (polar) directions in black (red). In each case, we plot the $v_{e}$ (solid line), the $\bar{v}_{e}$ (dashed line), and the " $v_{\mu}$ " (dash-dotted line) neutrinos. For comparison with the predictions of the leakage scheme of Ruffert et al. (1996), we have implemented their formalism, following exactly the description given in their paper in their Appendices $\mathrm{A}$ and $\mathrm{B}$. We plot the corresponding results at $10 \mathrm{~ms}$ intervals for each case (diamonds: $v_{e}$; triangles: $\bar{v}_{e}$; squares: " $v_{\mu}$ "; see also Section 4.1).

For the BNS merger models with initially no spins, co-rotating spins, and counter-rotating spins, the neutrino signal predicted by VULCAN/2D follows a qualitatively similar evolution. The initial fast rise represents the time it takes the neutrino emission processes to ramp up to their equilibrium rates, modulated by the diffusion time out of the opaque core (and out of the not-so-opaque surface layers) and the light-travel time of $\sim 1 \mathrm{~ms}$ to the radius of $200 \mathrm{~km}$ where the luminosity is recorded. The peak luminosity occurs at about $5 \mathrm{~ms}$ after the start of the VULCAN/2D simulations, and is dominated by $\bar{v}_{e}$ and " $v_{\mu}$ " neutrinos. The $v_{e}$ neutrino luminosity is initially systematically subdominant compared with the other two neutrino species. This is primarily a result of the high neutron richness of the merged object. Among the models, the stronger 


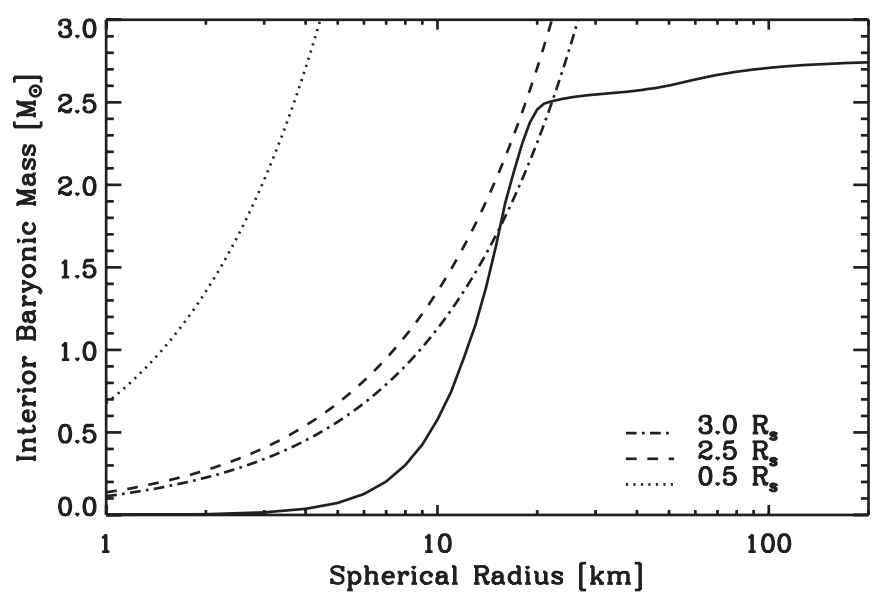

Figure 6. Interior baryonic mass vs. spherical radius for the BNS merger model with no initial spins. The time corresponding to these data is $60 \mathrm{~ms}$ after the start of the VULCAN/2D simulation. Following Ruffert et al. (1996), we overplot the Schwarzschild radius $R_{\mathrm{s}}(m(r))$, scaled by $0.5,2.5$, and 3, corresponding to the range of masses plotted on the vertical axis. Our results differ from those of Ruffert et al. (1996) in that only a small mass range falls within the radius $3 \times R_{\mathrm{S}}(m(r))$ of the last stable circular orbit for an equal mass binary in harmonic coordinates (Kidder et al. 1992; Wex \& Schaefer 1993). This difference likely results from the smaller mass binary system $\left(2 \times 1.4 M_{\odot}\right.$ here compared to $2 \times 1.65 M_{\odot}$ in their work; the mass is the baryonic mass in both cases here) and the stiffer EOS we employ (Shen EOS vs. Lattimer \& Swesty EOS), with an incompressibility of $180 \mathrm{MeV}$.

neutrino signal, in particular for the " $v_{\mu}$ " neutrinos, is produced in the counter-rotating model, which achieves the largest central temperature $(\sim 17 \mathrm{MeV})$, followed by the no-spin model $(\sim 15 \mathrm{MeV})$. The co-rotating spin model has the weakest neutrino signal of all three, mainly because the tidal locking leads to a very smooth merger and correspondingly low temperatures (the central temperature is about $10 \mathrm{MeV}$ ). Specifically, the angle-integrated, species-integrated, peak neutrino luminosity for the BNS model with no initial spin is $1.5 \times 10^{53}\left(2.2 \times 10^{52}\right.$, $7.0 \times 10^{52}$, and $6.7 \times 10^{52}$ ), for the co-rotating spin model $1.2 \times 10^{53}\left(2.2 \times 10^{52}, 5.7 \times 10^{52}\right.$, and $\left.3.9 \times 10^{52}\right)$, and for the counter-rotating spin model $2.2 \times 10^{53} \mathrm{erg} \mathrm{s}^{-1}\left(2.9 \times 10^{52}\right.$, $8.2 \times 10^{52}$, and $1.2 \times 10^{53}$ ). The values given in parentheses correspond in each case to the angle-integrated peak luminosity for the $v_{e}$, the $\bar{v}_{e}$, and the " $v_{\mu}$ " neutrinos, respectively. Apart from a significant discrepancy with the " $v_{\mu}$ "-neutrino luminosities, the peak neutrino luminosities using our MGFLD approach compare well with those published in the literature based on a leakage scheme (Ruffert et al. 1997; Rosswog \& Liebendörfer 2003). However, in all cases, cooling of the BNS merger causes a decrease of all neutrino luminosities after the peak (i.e., rather than a long-term plateau), with a faster decline rate in the no-spin and counter-rotating spin cases. The luminosity decay rate is faster for the " $v_{\mu}$ " neutrinos than for the electron-type neutrinos. This translates into a strong decrease of the neutrino-antineutrino annihilation rate due to its scaling with the square of the neutrino luminosity (see Section 4). In agreement with past work that focused on the initial 10-20 ms after the onset of coalescence, we find that the electron antineutrino luminosity dominates over the electron neutrino luminosity, but in contrast with the work of Ruffert et al. (1996) and Rosswog \& Liebendörfer (2003), our " $v_{\mu}$ " neutrino luminosities are at least one order of magnitude higher. This discrepancy may in part stem from their neglect of the nucleon-nucleon bremsstrahlung opacity and emission processes. Taking out the contribution from nucleon-nucleon bremsstrahlung in our VULCAN/2D BNS no-spin model leads
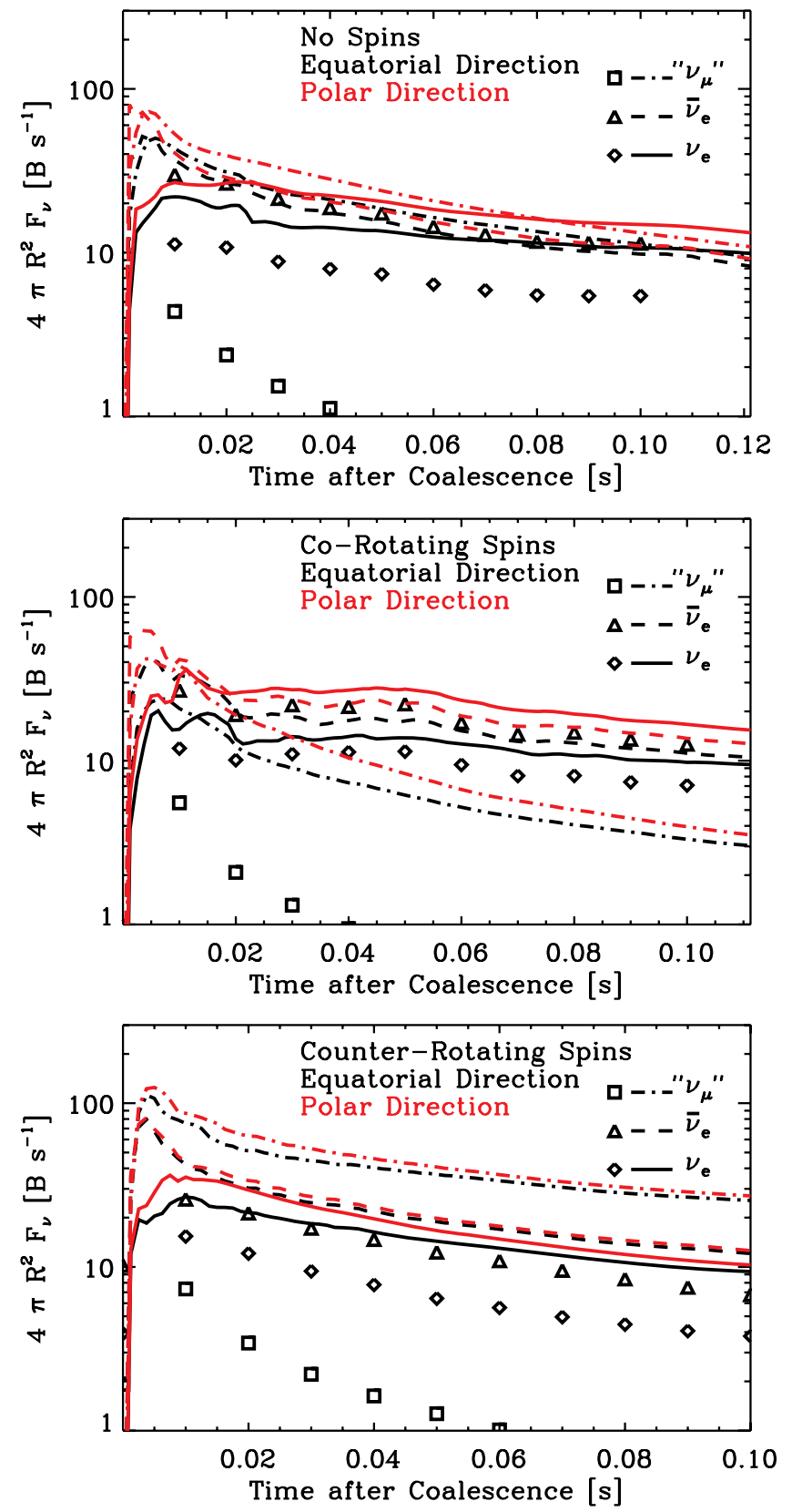

Figure 7. Time evolution of the MGFLD energy-integrated fluxes at $R=$ $200 \mathrm{~km}$, scaled by a factor $4 \pi R^{2}$ (thus equivalent to a luminosity), for the $v_{e}$ (solid), the $\bar{v}_{e}$ (dashed), and the " $v_{\mu}$ " (dash-dotted) neutrinos, plotted along the equatorial (black) and polar (red) directions, and for the BNS merger models initially with no spins (top), co-rotating spins (middle), and counterrotating spins (bottom). We also overplot the neutrino luminosities (diamonds: $v_{e}$; triangles: $\bar{v}_{e}$; squares: “ $v_{\mu}$ ") computed in Section 4.1 using the leakage scheme of Ruffert et al. (1996), but only for snapshots at $10 \mathrm{~ms}$ intervals. Nucleon-nucleon bremsstrahlung processes are not treated in their scheme and this is in part the origin of the very low " $v_{\mu}$ " neutrino luminosity compared to the VULCAN/2D prediction. Note that the fast rise of the VULCAN/2D neutrino luminosities reflects the light-travel time of $\sim 1 \mathrm{~ms}$ to the $200 \mathrm{~km}$ radius where the luminosity is recorded. Note that the luminosity unit used is the Bethe, i.e. $10^{51}$ erg $\equiv 1$ Bethe [1 B].

(A color version of this figure is available in the online journal.)

to a general reduction of the " $v_{\mu}$ " luminosity by a factor of $2-$ 3 , which then no longer dominates. However, it is still not as low as that predicted with our implementation of the leakage scheme of Ruffert et al. (1996, 1997). Moreover, in the no-spin and co-rotating spin cases, the relationships between the fluxes of the various neutrino flavors changes significantly with time, 
partly because they are differently affected by neutron star cooling and changes in neutron richness. While the decrease of the luminosity predicted by VULCAN/2D makes physical sense, it contrasts with the findings of Setiawan et al. $(2004,2006)$ in their study of neutrino emission from torus disks around $\sim 4 M_{\odot}$ black holes. In their models, they incorporate physical viscosity in the disk, together with the associated heat release, which keeps the gas temperature high. In our models, neutrino emission is a huge energy sink, not compensated by $\alpha$-disk viscosity heating.

The MGFLD treatment we use permits the calculation of the energy-dependent neutrino spectrum and its variation with angle. For the BNS merger models with initially no spins (left), co-rotating spins (middle), and counter-rotating spins (right), we show such spectra along the equatorial (black) and polar (red) directions in Figure 8, using a reference radius of $200 \mathrm{~km}$. We can also explicitly compute average neutrino energies, here defined as

$$
\sqrt{\left\langle\varepsilon_{\nu}^{2}\right\rangle} \equiv\left[\frac{\int d \varepsilon_{\nu} \varepsilon_{v}^{2} F_{\nu}\left(\varepsilon_{\nu}, R\right)}{\int d \varepsilon_{v} F_{\nu}\left(\varepsilon_{\nu}, R\right)}\right]^{\frac{1}{2}}
$$

At $50 \mathrm{~ms}$ after the start of the VULCAN/2D simulations, such average neutrino energies in the no-spin BNS model and along the pole are $13.4\left(v_{e}\right), 16.7\left(\bar{v}_{e}\right)$, and $25.2 \mathrm{MeV}$ (" $v_{\mu}$ "), while they are 10.7, 15.6, and $21.2 \mathrm{MeV}$, respectively, along the equatorial direction. In the co-rotating model and along the polar direction, we have $14.0\left(v_{e}\right), 17.0\left(\bar{v}_{e}\right)$, and $22.0 \mathrm{MeV}$ (" $v_{\mu}$ "), and along the equatorial direction, we have, respectively 9.9, 15.2, and 17.5 MeV. In the counter-rotating model and along the polar direction, we have $12.9\left(v_{e}\right), 17.1\left(\bar{v}_{e}\right)$, and $27.5 \mathrm{MeV}$ (" $v_{\mu}$ "), and along the equatorial direction, we have 11.5, 16.7, and $25.1 \mathrm{MeV}$, respectively. The oblateness of the compact massive BNS merger, with its lower temperature and its more gradual density fall-off along the equator, systematically places the neutrinospheres in lower temperature regions, translating into softer neutrino spectra.

The aspherical density/temperature distributions naturally lead to a strong anisotropy of the radiation field, whose latitudinal dependence is shown in Figure 9 for the three models (we use the same left to right ordering), and at a time of $60 \mathrm{~ms}$ after the start of the VULCAN/2D simulations. Note the stronger neutrino flux along the polar direction (see also Rosswog \& Liebendörfer 2003, their Figure 12), resulting from the larger radiating surface seen from higher latitudes, as well as the systematically larger matter temperatures at the decoupling region along the local vertical (also yielding a harder neutrino spectrum). In Figure 9, we include in red the corresponding neutrino fluxes computed with the $16 \vartheta$-angle $S_{n}$ scheme, which shows comparable fluxes along the equator, but considerably larger ones at higher latitudes. We also show in Figure 10 the energyand species-integrated specific neutrino energy-deposition and loss rates in the no-spin BNS model at $60 \mathrm{~ms}$ after the start of the simulation, for both the MGFLD and the $S_{n}$ calculations. Note how much more emphasized the anisotropy is with the multiangle, $S_{n}$, solver, and how enhanced is the magnitude of the deposition along the polar direction. This is a typical property seen for fast-rotating PNSs simulated with such a multiangle solver (Ott et al. 2008).

In Figure 11, we show the energy-dependent neutrinosphere radii for the $v_{e}$ (left), $\bar{v}_{\mathrm{e}}$ (middle), and " $v_{\mu}$ " (right) neutrinos, and for the BNS merger models with initially no spins (top row), co-rotating spins (middle row), and counter-rotating spins
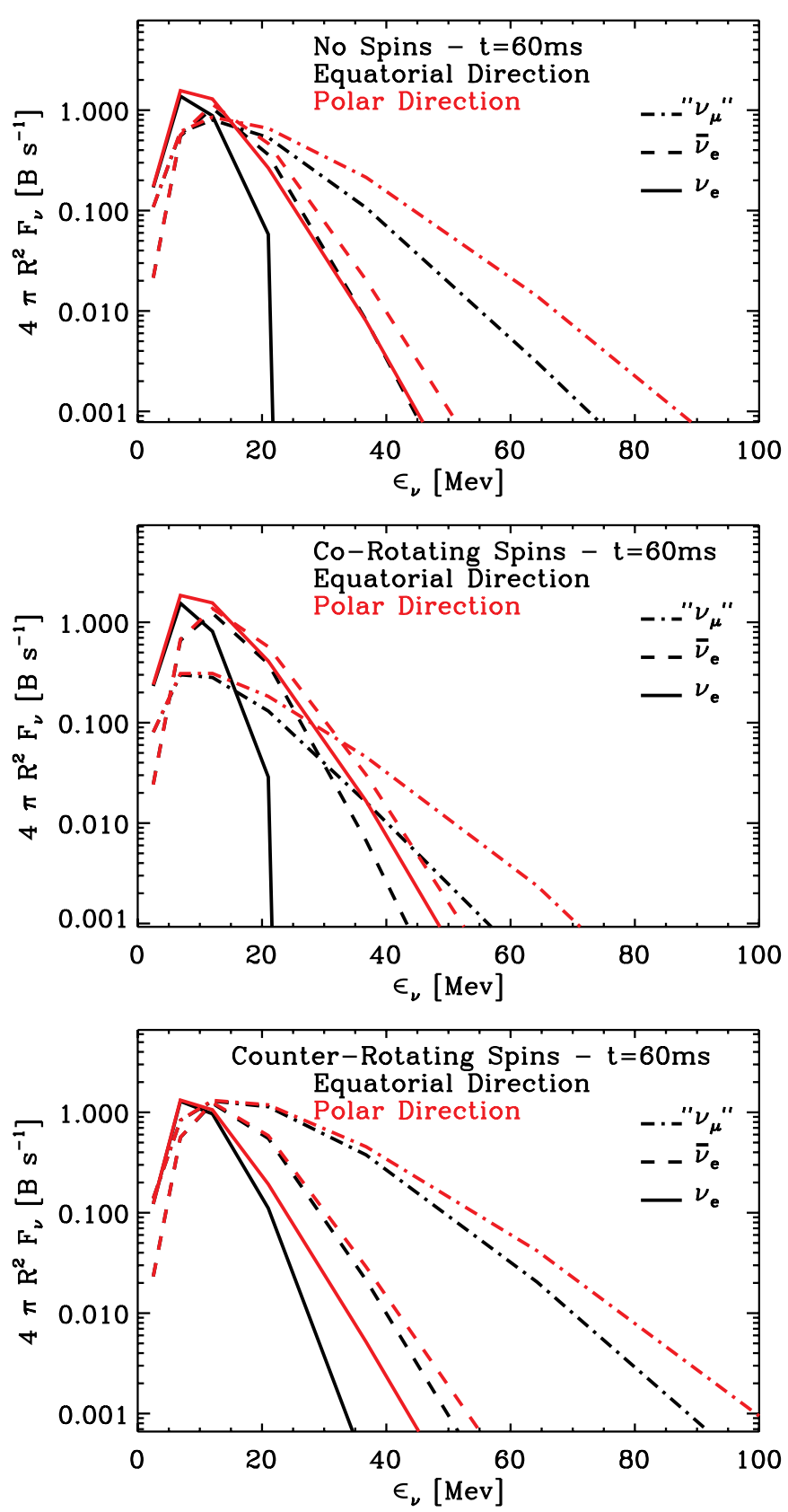

Figure 8. MGFLD flux spectra at $R=200 \mathrm{~km}$, scaled by a factor $4 \pi R^{2}$ (thus equivalent to a luminosity spectrum), for $v_{e}$ (solid), $\bar{v}_{e}$ (dashed), and " $v_{\mu}$ " (dash-dotted) neutrinos, for the BNS merger models initially with no spins (left), co-rotating spins (middle), and counter-rotating spins (right), and plotted along the equatorial (black) and polar (red) directions. For all models, the time corresponds to $60 \mathrm{~ms}$ after the start of the simulation.

(A color version of this figure is available in the online journal.)

(bottom row). These decoupling radii manifest a strong variation with latitude, but also with energy due to the approximate $\varepsilon_{v}^{2}$ dependence of the material opacity to neutrinos. The diffusion of neutrinos out of the opaque core, which leads to global cooling of the neutron star, is also energy dependent. The locationdependent diffusion timescale $t_{v_{i}}^{\text {diff }}(r, z)$ is given by (Mihalas \& Mihalas 1984; Ruffert et al. 1996)

$$
t_{v_{i}}^{\mathrm{diff}}(r, z) \approx 3 \tau_{v_{i}} \Delta R / c,
$$

where $c$ is the speed of light, $\Delta R$ is the local density scale height, $\tau_{v_{i}}$ is the optical depth (species and energy dependent), 

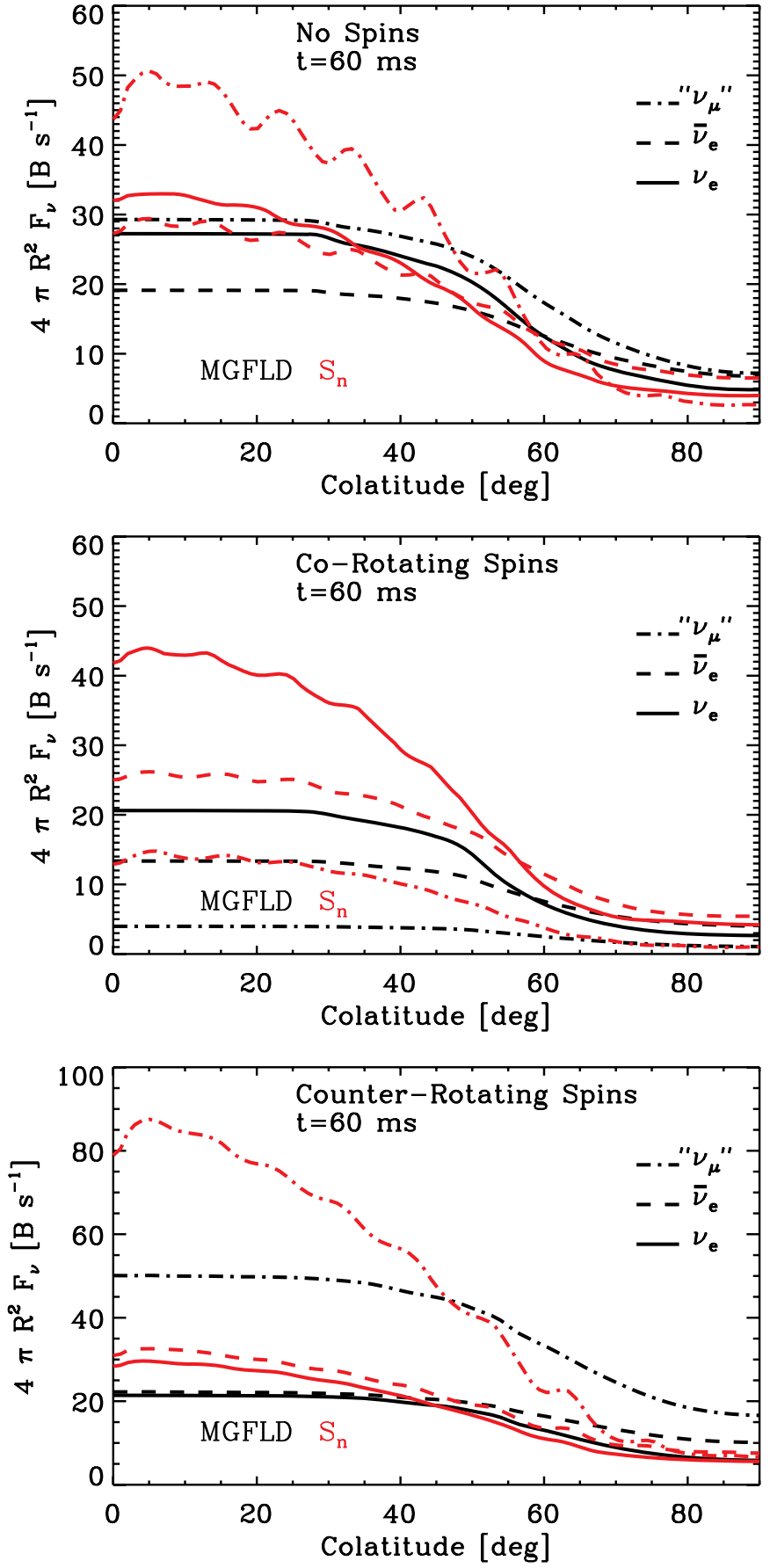

Figure 9. Angular variation from pole to equator of the energy-integrated neutrino fluxes at $100 \mathrm{~km}$ (this radius is chosen smaller than for Figure 7 to better reveal the flux anisotropy), scaled by a factor $4 \pi R^{2}$ (thus equivalent to a luminosity), for the $v_{e}$ (solid), the $\bar{v}_{e}$ (dashed), and the " $v_{\mu}$ " (dash-dotted) neutrinos, for the BNS merger models initially with no spins (top), co-rotating spins (middle), and counter-rotating spins (bottom). For each model, we show the MGFLD (black) and $S_{n}$ (red; using the $16 \vartheta$-angle calculations) predictions at $60 \mathrm{~ms}$ after the start of each simulation. Note the $S_{n}$ oscillatory features in the neutrino flux, more prominent along the polar direction, which is an artifact of the $S_{n}$ scheme. The range of the ordinate axis varies between frames.

(A color version of this figure is available in the online journal.)

and $v_{i}$ is the neutrino species (i.e., $v_{e}, \bar{v}_{e}$, or " $v_{\mu}$ "). For the $v_{e}$ neutrinos, we find an optical depth in the core that varies from a few $\times 10^{2}$ for $10 \mathrm{MeV}$ neutrinos to a few $\times 10^{4}$ for $100 \mathrm{MeV}$ neutrinos. These optical depths translate into diffusion times that are on the order of $10 \mathrm{~ms}$ to $1 \mathrm{~s}$. The quasi-Keplerian disk that extends from $20-30$ to $100 \mathrm{~km}$ is moderately optically

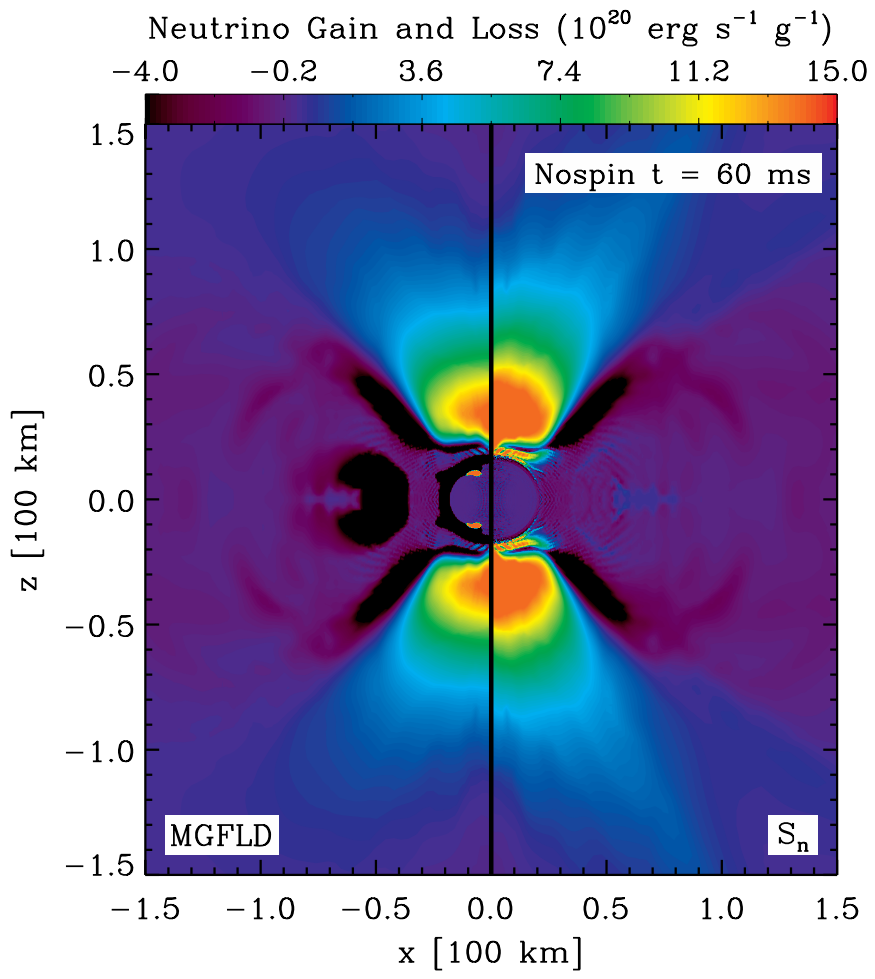

Figure 10. Colormaps of energy- and species-integrated specific neutrino energy deposition (whose volume-integrated values is referred to as $Q(\mathrm{cc})$; see Figure 17) and loss rates in the BNS merger model with no initial spin (shown here in units of $10^{20} \mathrm{erg} \mathrm{s}^{-1} \mathrm{~g}^{-1}$ ). This snapshot corresponds to a time of $60 \mathrm{~ms}$ after the start of the simulation. The left section of the plot depicts the MGFLD result and the right shows the result of the $S_{n}$ calculation. Note the distinctively enlarged polar gain regions and greater specific gain of the $S_{n}$ result compared to the MGFLD calculation. This is in part a consequence of the larger polar neutrino fluxes and overall greater flux asymmetry in the $S_{n}$ model (see Figure 9).

(A color version of this figure is available in the online journal.)

thick to neutrinos at the peak of the energy distribution (i.e., at 10-20 MeV). Heat can thus leak out in the vertical direction over a typical diffusion time of a few tens of milliseconds (see Figure 12). This is comparable to the accretion timescale of the disk itself (Setiawan et al. 2004, 2006), which suggests that this neutrino energy is available to power relativistic ejecta, and is not advected inward with the gas, as proposed by Di Matteo et al. (2002).

Compared to PNSs formed in the "standard" core-collapse of a massive star, SMNSs formed through coalescence have a number of contrasting properties. (1) most of the material is at nuclear density, unlike in PNSs where newly accreted material deposited at the PNS surface radiates its thermal energy as it contracts and cools. (2) most of the material is neutron rich, thereby diminishing the radiation of electrontype neutrinos in favor of antielectron-type neutrinos. In PNSs, electron-type neutrinos are an important cooling agent, but have large opacities. In SMNSs, the material is strongly deleptonized and electron-type neutrinos are nondegenerate. They suffer relatively lower opacity and can therefore diffuse out more easily. The opacity of other neutrino species is even lower and therefore more prone to energy leakage through radiation. (3) energy gain through accretion is modest since there is merely a few $0.1 M_{\odot}$ of material outside of the SMNS. In our three merger models, the internal energy budget of such shear-heated SMNSs is $\gtrsim 10^{53} \mathrm{erg}$, half of which is thermal energy. With a total neutrino luminosity of $\sim 5 \times 10^{52} \mathrm{erg} \mathrm{s}^{-1}$, the cooling 

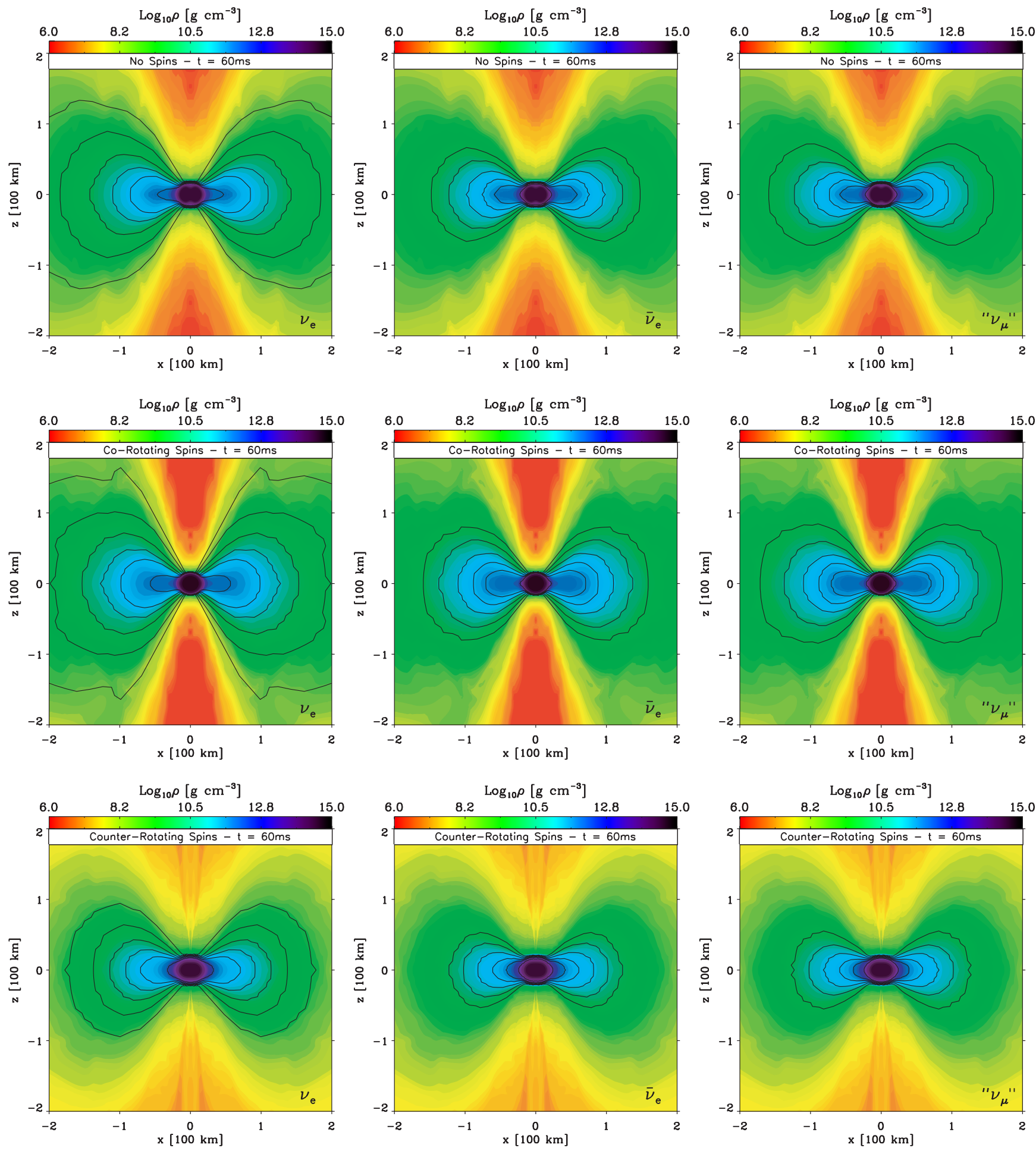

Figure 11. Using a colormap of the density distribution (as shown in log scale) at $60 \mathrm{~ms}$ after the start of the VULCAN/2D simulations for the BNS merger models with initially no spins (top row), co-rotating spins (middle row), and counter-rotating spins (bottom row), we overplot in each case the contours corresponding to the energy-dependent and latitude-dependent neutrinosphere radii at neutrino energies $\varepsilon_{v}$ of 2.50, 6.87, 12.02, 21.01, 36.74, 64.25 MeV, for the $v_{e}\left(\right.$ left column), $\bar{v}_{\mathrm{e}}$ (middle column), and " $v_{\mu}$ " (right column) neutrinos. Corresponding radii (along a given latitude) increase monotonically with energy (matter opacity to neutrinos scales as $\sim \varepsilon_{v}^{2}$ ). For these calculations, the optical depth is integrated inward along a fixed latitude starting at the maximum spherical radius of $3000 \mathrm{~km}$.

(A color version of this figure is available in the online journal.)

timescale for these SMNS should be on the order of a second. This is significantly, but not dramatically, shorter than the 10 30 s cooling timescale of hot PNSs, i.e., the time it takes these objects to radiate a total of $\sim 3 \times 10^{53} \mathrm{erg}$ of internal energy.
A fraction of the diffusing core energy is absorbed through charge-current reactions (with associated volume-integrated energy $\dot{E}(\mathrm{cc}))$ and turned into internal energy in a "gain" layer at the surface of the SMNS, primarily on the pole-facing side and at high latitudes (see Figure 10). This neutrino energy deposition 


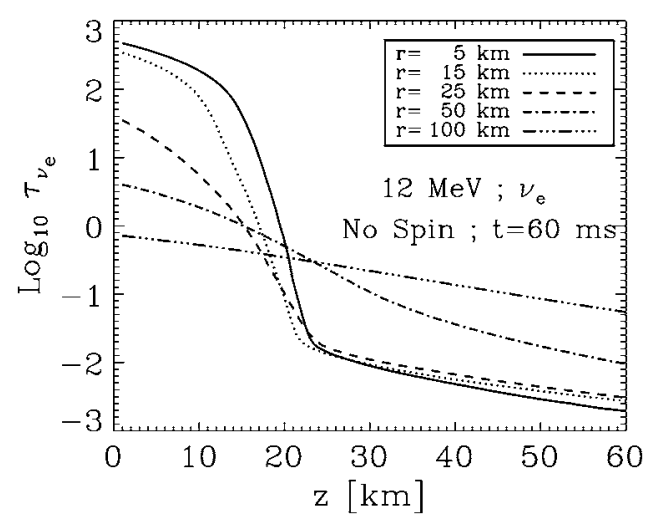

Figure 12. Variation of the log of the optical depth along the $z$-direction for the $v_{e}$ neutrino at $12.02 \mathrm{MeV}$ in the BNS merger model with no initial spins, plotted for a selection of cylindrical radii: 5, 15, 25, 50, and $100 \mathrm{~km}$ (see legend for the corresponding linestyles). The time corresponds to $60 \mathrm{~ms}$ after the start of the VULCAN/2D simulations. Note that the disk transitions from optically thick to optically thin conditions at $\sim 100 \mathrm{~km}$, and that optical depth increases to a few hundreds of km only inside the SMNS. For most of the disk, the diffusion time (Equation 2) for $12.02 \mathrm{MeV} v_{e}$ neutrinos is on the order of a few milliseconds. The disk is therefore only moderately optically thick for neutrinos with energies at the peak of the spectral energy distribution.

is the origin of a thermally driven wind whose properties we now describe.

\subsection{Neutrino-Driven Wind}

A few milliseconds after the start of our simulations and onset of the burst of neutrinos from the BNS merger, a neutrinodriven wind develops. It relaxes into a quasi-steady state by the end of the simulations at $100 \mathrm{~ms}$. Note that in this quasi steady-state configuration, and as shown in Figure 10, the energy gain/losses due to neutrino in the polar direction is mostly net heating, with no presence of a sizable net cooling region. In Figure 13, we show this evolution for the no-spin BNS merger model for four selected times: 10, 30, 60, and $100 \mathrm{~ms}$. One can see the initial transient feature, which advects out and eventually leaves the grid. This transient phase is not caused by neutrino energy deposition, but is rather due to infall at small radii and in the polar regions of the ambient medium that we placed around the merged object, as well as due to the sound waves generated by the core during the initial quakes. One can then see the strengthening neutrino-driven wind developing in the polar funnel. The side lobes of the SMNS confine the ejecta at radii $\lesssim 500 \mathrm{~km}$ to a small angle of $\sim 20^{\circ}$ about the poles, but this opening angle grows at larger distances to $\sim 90^{\circ}$. The confinement at small radii also leads to the entrainment of material from the side lobes, overloading the wind and making it choke. The radial density/velocity profile of this wind flow is thus kinked, with variations in velocity that can be as large as the average asymptotic velocity value of $\sim 30,000 \mathrm{~km} \mathrm{~s}^{-1}$ (Figure 4). As shown in Figure 13, the mass loss associated with the neutrino-driven wind is on the order of a few $10^{-3} M_{\odot} \mathrm{s}^{-1} \mathrm{str}^{-1}$ at a few tens of milliseconds, but decreases to $10^{-3}-10^{-4} M_{\odot} \mathrm{s}^{-1}$ $\mathrm{str}^{-1}$ at $100 \mathrm{~ms}$. The wind is also weaker along the poles than along the $70^{\circ}-80^{\circ}$ latitudes. The associated angle-integrated mass loss summed over $100 \mathrm{~ms}$ approaches $10^{-4} M_{\odot}$ and is made up in part of high $Y_{\mathrm{e}}$ material $(\sim 0.5)$ along the pole, but mostly of low $Y_{\mathrm{e}}$ material $(\sim 0.1-0.2)$ along midlatitudes. Thus, "r-process material" will feed the interstellar medium through this neutrino-driven wind. This latitudinal variation of the electron fraction at large distances is controlled by the relative strength of the angle- and time-dependent $v_{e}$ and $\bar{v}_{e}$ neutrino luminosities, the expansion timescale of the "wind" parcels, and the neutron richness at their launching site (Ott et al. 2007). Along the pole and at the SMNS surface, the low-density, high neutron richness, and relatively stronger $v_{e}$ neutrino luminosities at late times in the no-spin and co-rotating spin models lead to a high asymptotic $Y_{\mathrm{e}}$ value (high proton richness). Despite the relatively high resolution employed in our simulations $(\sim 300 \mathrm{~m}$ in the radial direction at $\sim 20 \mathrm{~km})$, higher resolution would be needed to resolve this region accurately. Although we expect this trend would hold at higher resolution, it would likely yield lower asymptotic values of the electron fraction along the pole.

Along the equatorial direction, low $Y_{\mathrm{e}}$ material $(\sim 0.1-0.2)$ migrates outward, but its velocity is below the local escape speed and it is unclear how much will eventually escape to infinity. ${ }^{8}$ This is further illustrated in Figure 14 where we show the angular variation of the density and velocity at $2900 \mathrm{~km}$ in the no-spin BNS model, at $121 \mathrm{~ms}$. The BNS merger is thus cloaked along the poles by material with a density in excess of $10^{4} \mathrm{~g} \mathrm{~cm}^{-3}$, while along lower latitudes even denser material from the side lobes obstructs the view from the center of the SMNS. Importantly, wind material will feed the polar regions for as long as the merger remnant remains gravitationally stable. Being so heavily baryon-loaded, the outflow can in no way be accelerated to relativistic speeds with high Lorentz factors. In this context, the powering of a short-hard GRBs is impossible before black hole formation.

As seen in Figure 5, the rotational profile in the inner $100 \mathrm{~km}$ is strongly differential in the no-spin and counterrotating spin cases, while it is quasi-uniform in the corotating spin case (see also Rosswog \& Davies 2002, their Figure 17). The good conservation of specific angular momentum in VULCAN/2D is in part responsible for the preservation of the initial rotation profile throughout the simulation. In reality, such a differential rotation should not survive. Our two-dimensional axisymmetric setup inhibits the development of triaxial instabilities that arise at modest and large ratios of rotational and gravitational energies (Rampp et al. 1998; Centrella et al. 2001; Saijo et al. 2003; Ott et al. 2005, 2007), i.e., under the conditions that prevail here. Moreover, our good, but not excellent, spatial resolution prevents the modeling of the magnetorotational instability, whose effect is to efficiently redistribute angular momentum (Balbus \& Hawley 1991b; Pessah et al. 2006, 2007), and dissipate energy, and, in the present context, leads to mass accretion onto the SMNS. The magneto-rotational instability, operating on an rotational timescale, could lead to solid-body rotation within a few milliseconds in regions around $\sim 10 \mathrm{~km}$, and within a few tens of milliseconds in regions around $\sim 100 \mathrm{~km}$. Note that this is a more relevant timescale than the typical $\sim 100 \mathrm{~s}$ that characterizes the angular-momentum loss through magnetic dipole radiation (Rosswog \& Davies 2002). Importantly, in the three BNS merger models we study, we find that the free energy of rotation (the energy difference between the given differentially rotating object and that of the equivalent solid-body rotating object with the same cumulative angular momentum) is very large. Despite differences of a factor of a few between models, it typically reaches $\sim 5 \times 10^{51}$ erg inside the SMNS (regions with densities greater than $10^{14} \mathrm{~g} \mathrm{~cm}^{-3}$ ), but

\footnotetext{
8 Note that in the counter-rotating model, an axis problem in the form of a low-density, high-velocity, narrow region starts at the onset of the neutrinodriven wind and persists throughout the simulation. This spurious feature is, however, localized and therefore does not influence the global properties of the simulation.
} 

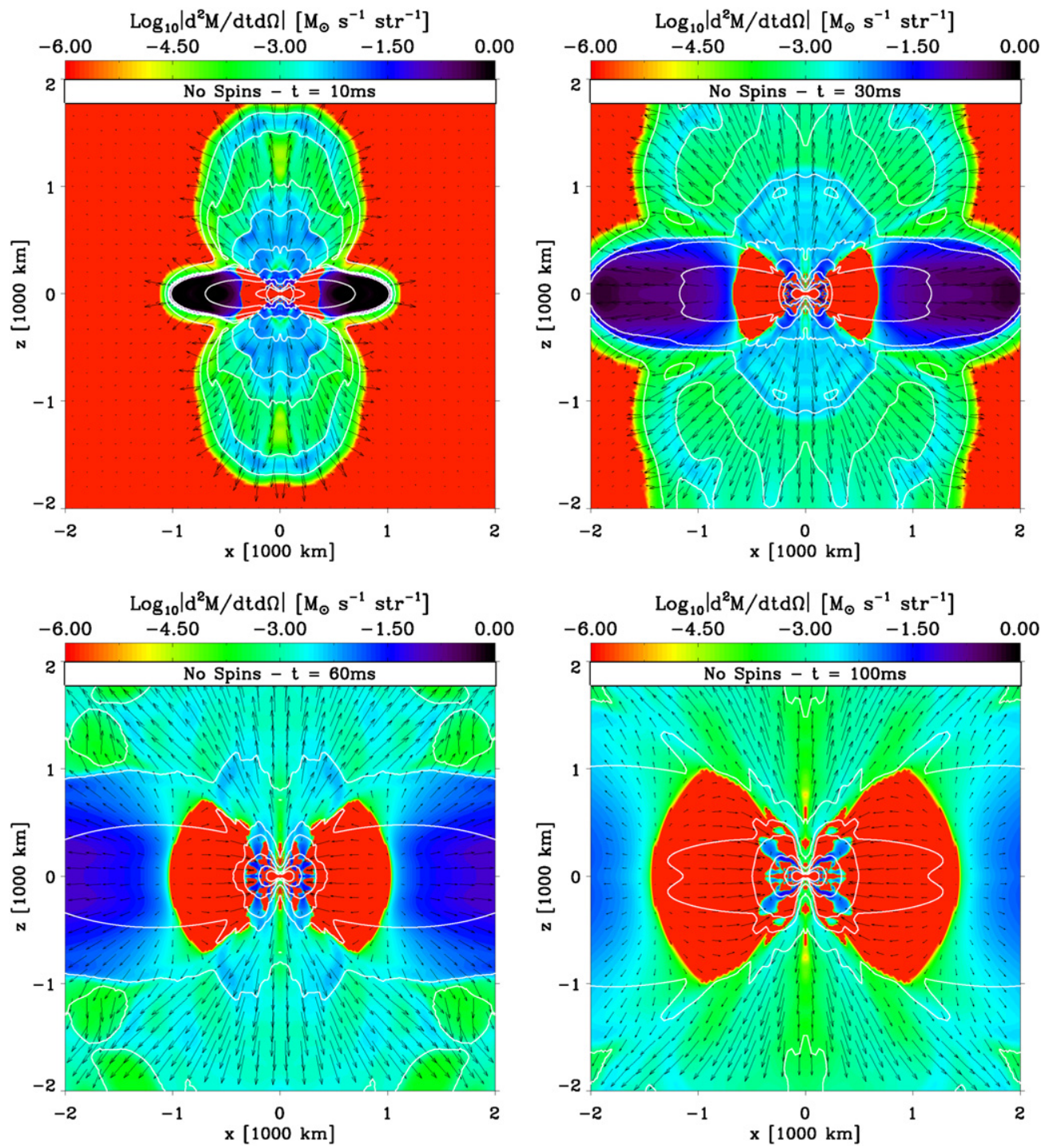

Figure 13. Colormaps of the log of the mass-loss rate per steradian $\left(d^{2} M / d t d \Omega\right.$, in units of $\left.M_{\odot} \mathrm{s}^{-1} \mathrm{str}^{-1}\right)$ for the no-spin BNS merger model at $10 \mathrm{~ms}$ (top left), $30 \mathrm{~ms}$ (top right), $60 \mathrm{~ms}$ (bottom left), and $100 \mathrm{~ms}$ (bottom right) after the start of the VULCAN/2D simulation, and depicting the mass loss associated with the initial transient, followed by the neutrino-driven wind. The displayed region covers $2000 \times 2000 \mathrm{~km}^{2}$. Regions that are infalling or denser than $10^{10} \mathrm{~g} \mathrm{~cm}{ }^{-3}$ are shown in red, and velocity vectors, overplotted in black, have a length saturated at $7 \%$ of the width of the display for a magnitude of $30,000 \mathrm{~km} \mathrm{~s}^{-1}$. Note the concomitant mass loss from the poles down to midlatitudes (the wind) and the expansion of BNS merger material at near-equatorial latitudes.

(A color version of this figure is available in the online journal.)

is on the order of $2 \times 10^{52} \mathrm{erg}$ in the torus disk, regions with densities between $10^{11}$ and $10^{14} \mathrm{~g} \mathrm{~cm}^{-3}$. Similar conditions in the core-collapse context yield powerful, magnetically (and thermally) driven explosions (LeBlanc \& Wilson 1970; BisnovatyiKogan et al. 1976; Akiyama et al. 2003; Ardeljan et al. 2005; Moiseenko et al. 2006; Obergaulinger et al. 2006; Burrows et al. 2007a; Dessart et al. 2007). Rotation dramatically enhances the rate of mass ejection by increasing the density rather than the velocity of the flow, even possibly halting accretion and inhibiting the formation of a black hole (Dessart et al. 2008). In the present context, the magneto-rotational effects, which we do not include here, would considerably enhance the mass flux of the neutrino-driven wind. Importantly, the loss of differential rotational energy needed to facilitate the gravitational instability is at the same time delaying it through the enhanced mass loss it induces. Work is needed to understand the systematics of this interplay, and how much rotational energy the back hole is eventually endowed with.
Oechslin et al. (2007), using a conformally flat approximation to GR and an SPH code, find that BNS mergers of the type discussed here and modeled with the Shen EOS avoid the general-relativistic gravitational instability for many tens of milliseconds after the neutron stars first come into contact. Baumgarte et al. (2000), and more recently Morrison et al. (2004), Duez et al. (2004, 2006), and Shibata et al. (2006), using GR (and for some using a polytropic EOS), find that imposing even modest levels of differential rotation yields a significant increase by up to $50 \%$ in the maximum mass that can be supported stably, in particular pushing this value beyond that of the merger remnant mass after coalescence. Surprisingly, Baiotti et al. (2008), using a full GR treatment but with a simplified (and soft) EOS, find prompt black hole formation in such high-mass progenitors. Despite this lack of consensus, the existence of neutron stars with a gravitational mass around $2 M_{\odot}$ favors a high incompressibility of nuclear matter, such as in the Shen EOS, and suggests that SMNSs formed through BNS merger events may survive for tens of milliseconds before 


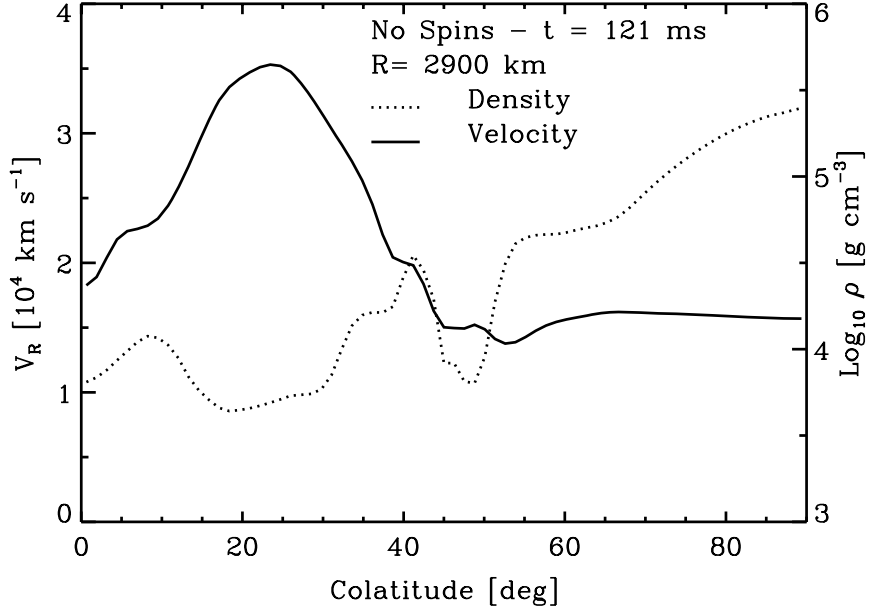

Figure 14. Variation of the radial velocity (solid) and density (dotted; log scale) vs. colatitude and at a spherical radius of $2900 \mathrm{~km}$ for the BNS merger model with no initial spin. The time plotted is $121 \mathrm{~ms}$ after the start of the MGFLD VULCAN/2D simulation. Note that the escape speed at this radius and for a $\sim 2.8 M_{\odot}$ central object is $\sim 16,000 \mathrm{~km} \mathrm{~s}^{-1}$, so most of the mass near-equatorial latitudes will remain trapped in the gravitational potential of the SMNS.

experiencing the general-relativistic gravitational instability. In particular, the presence of a significant amount of material (a few tenths of a solar mass), located on wide orbits in a Keplerian disk, reduces the amount of mass that resides initially in the core, i.e., prior to outward transport of angular momentum.

\section{4. $v-\bar{v}$ ANNIHILATION}

Besides being prime candidates for gravitational wave emission, BNS mergers may lead to short-hard GRBs (for an overview, see, e.g., Piran 2005; Nakar 2007). In this context, the high energy radiation arises from nonthermal radiation associated with relativistic ejecta. The annihilation of neutrino pairs, by the process

$$
v_{i}+\bar{v}_{i} \rightarrow e^{+}+e^{-} ; \quad i \in\{e, \mu, \tau\},
$$

represents one possible powering source for high-Lorentz-factor baryon-free ejecta beamed into a small solid angle about the rotation axis of the BNS merger (Ruffert et al. 1997; Ruffert \& Janka 1999; Asano \& Fukuyama 2000, 2001; Miller et al. 2003; Kneller et al. 2006; Birkl et al. 2007; Rosswog et al. 2003; Rosswog 2005, and references therein).

Here, we revisit this proposition by quantitatively studying the energetics of neutrino-antineutrino annihilation in the three BNS mergers that we simulated with VULCAN/2D. First, in Section 4.1, we apply the approach used in previous work and based on the leakage scheme of Ruffert et al. (1996, 1997). This approach was also used by Rosswog \& Liebendörfer (2003), but for merger events whose dynamical evolution was computed independently. ${ }^{9}$ Note that similar $v_{i} \bar{v}_{i}$ annihilation rate calculations, i.e., without the full momentum-space angular dependence, have been carried out in the core-collapse and PNS contexts, both with Newtonian gravity (Goodman et al. 1987; Cooperstein et al. 1986, 1987; Janka 1991) and in GR (Salmonson \& Wilson 1999; Bhattacharyya et al. 2007). In Section 4.2 we present a new formalism based on moments of the neutrino-specific intensity. The angle and energy dependence of the neutrino radiation field is obtained by post-processing

\footnotetext{
9 SPH versus grid-based code; different EOS; different resolution.
}

individual MGFLD VULCAN/2D snapshots (see Section 3) with the multiangle, $S_{n}$, variant. In both cases, because the power associated with neutrino-antineutrino annihilation is subdominant compared to that associated with the chargecurrent reactions prior to black hole formation, it can be estimated only through a post-processing step.

\subsection{Formalism Based on a Beakage Scheme and the Approach of Ruffert et al. (1996)}

Our first approach to estimate the neutrino-antineutrino annihilation rate is based on a leakage scheme. We implemented the method presented by Ruffert et al. (1996, 1997), which comprises two steps. First, using the processes described in Section 3.1, the instantaneous rate of neutrino emission $Q\left(v_{i}\right)$ is computed for all grid cells. It is subsequently weighted by the direction-dependent factor that depends on the radiativediffusion timescale $t_{v_{i}}^{\text {diff }}$ and neutrino-emission timescale $t_{v_{i}}^{\text {loss }}$ relevant for that cell. Since we are primarily interested in the energy deposition in the polar regions, we consider only the cylindrical-z-direction when estimating $t_{v_{i}}^{\text {diff }}$. The effective emissivity $Q^{\text {eff }}\left(v_{i}\right)$ then has the form

$$
Q^{\text {eff }}\left(v_{i}\right)=\frac{Q\left(v_{i}\right)}{1+\left(t_{v_{i}}^{\text {loss }}\right)^{-1} t_{v_{i}}^{\text {diff }}},
$$

where expressions for the various components are given explicitly in Appendices A and B of Ruffert et al. (1996).

In Figure 15, we show the effective emissivity resulting from this leakage scheme and with the neutrino processes of Ruffert et al. (1996) for the $v_{e}$ (left column), $\bar{v}_{e}$ (middle column), and " $v_{\mu}$ " (right column) neutrinos, for the BNS merger models with initially no spins (top row), co-rotating spins (middle row), and counter-rotating spins (bottom row). For each process, emission is conditioned by the competing elements of optical depth, density, temperature, and electron fraction. In practice, it peaks in regions that are dense, hot, but not too optically thick, i.e., at the surface of the SMNS (see density contours in Figure 15, overplotted in black). Optical depths in excess of 100 for all neutrino energies make the inner region (the inner $\sim 15 \mathrm{~km}$, where densities have nuclear values) a weak "effective" emitter, with their contribution operating on a $0.1-1 \mathrm{~s}$ timescale. The dominant emission associated with the " $v_{\mu}$ " neutrinos originates from a considerably higher-density region than that associated with the electron-type neutrinos, i.e. $10^{12}-10^{13} \mathrm{~g} \mathrm{~cm}^{-3}$ compared to $10^{9}-10^{11} \mathrm{~g} \mathrm{~cm}^{-3}$, with a corresponding "leakage" luminosity in all three merger models that is typically an order of magnitude smaller than predicted by VULCAN/2D (see Figure 7). This low " $v_{\mu}$ " emissivity is likely caused by the neglect of nucleon-nucleon bremsstrahlung processes in the approach of Ruffert et al. $(1996,1997)$, which leads to a smaller decoupling radius for " $v_{\mu}$ " neutrinos, and, therefore, an underestimate of the size of the radiating surface from which they emerge (as shown in Figure 11, right column). In practice, the decoupling radius is energy dependent, but here we stress the systematic reduction of the decoupling radius for all " $v_{\mu}$ " neutrino energies because of the neglect of this extra opacity source. The importance of the bremsstrahlung process for " $v_{\mu}$ " emissivity and opacity has been emphasized by Thompson et al. (2000) for "hot" PNSs. The SMNS that results from the merger is considerably heated by shocks and shear, and is thus also in a configuration where such bremsstrahlung processes are important and should be included. 

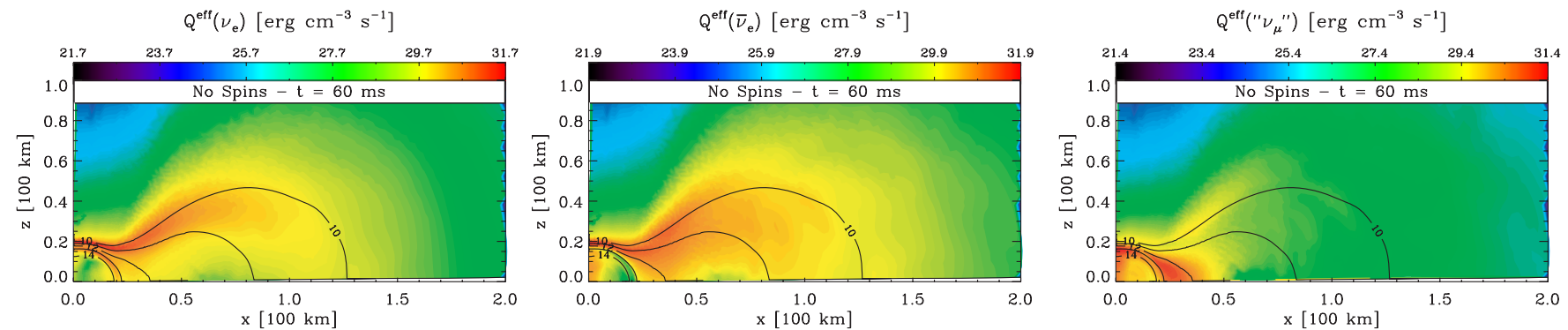

$\mathrm{Q}^{\mathrm{eff}}\left(\nu_{\mathrm{e}}\right)\left[\mathrm{erg} \mathrm{cm} \mathrm{cm}^{-3} \mathrm{~s}^{-1}\right]$
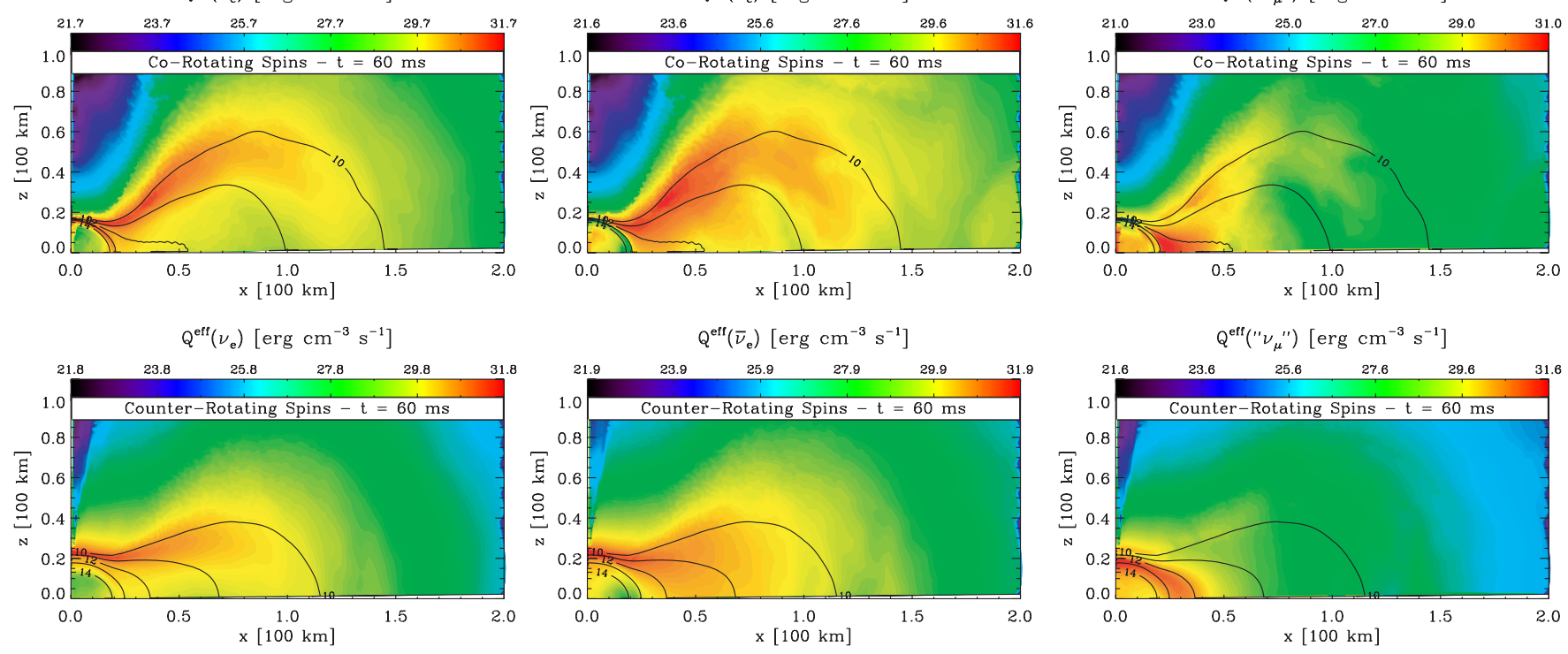

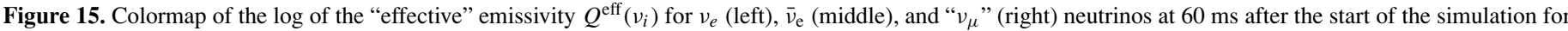

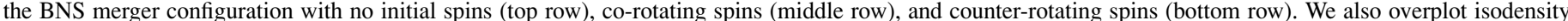

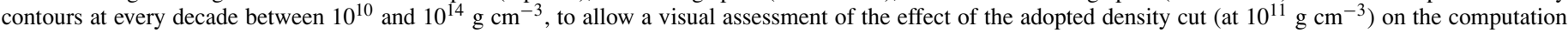

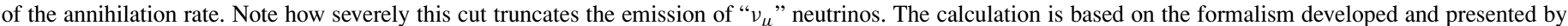
Ruffert et al. (1996).

(A color version of this figure is available in the online journal.)

From the effective emissivity distribution computed for each neutrino flavor with the leakage scheme, Ruffert et al. (1997) then sum the contributions from all pairings between grid cells. For completeness, we briefly reproduce here the presentation of Ruffert et al. (1997). The integral to be computed for the energy-integrated $v_{i} \bar{v}_{i}$ (representing equivalently $v_{e} \bar{v}_{\mathrm{e}}$ or " $v_{\mu} \bar{v}_{\mu}$ ") annihilation rate at position $\vec{r}$ is

$$
\begin{aligned}
Q^{+}\left(\nu_{i} \bar{v}_{i}\right)= & \frac{1}{4} \frac{\sigma_{0}}{c\left(m_{e} c^{2}\right)^{2}}\left\{\frac{\left(C_{1}+C_{2}\right)_{v_{i} \bar{v}_{i}}}{3} \cdot \oint_{4 \pi} d \Omega I_{\nu_{i}}\right. \\
& \times \oint_{4 \pi} d \Omega^{\prime} I_{\bar{v}_{i}}\left[\langle\epsilon\rangle_{v_{i}}+\langle\epsilon\rangle_{\bar{v}_{i}}\right](1-\cos \Phi)^{2} \\
& +C_{3, v_{i} \bar{v}_{i}}\left(m_{e} c^{2}\right)^{2} \cdot \oint_{4 \pi} d \Omega I_{v_{i}} \\
& \left.\times \oint_{4 \pi} d \Omega^{\prime} I_{\bar{v}_{i}} \frac{\langle\epsilon\rangle_{v_{i}}+\langle\epsilon\rangle_{\bar{v}_{i}}}{\langle\epsilon\rangle_{v_{i}}\langle\epsilon\rangle_{\bar{v}_{i}}}(1-\cos \Phi)\right\} .
\end{aligned}
$$

$I_{\nu_{i}}$ is the neutrino-specific intensity. $\Omega$ and $\Omega^{\prime}$ are the solid angles subtended by the cells producing the neutrino and antineutrino radiation incident from all directions. $C_{1}, C_{2}$, and $C_{3}$ are related to the weak coupling constants, $C_{\mathrm{A}}$ and $C_{\mathrm{V}}$, and depend on the neutrino species (see Ruffert et al. 1997 as well as Section 4.2). $\sigma_{0}$ is the baseline weak interaction cross section, $1.705 \times 10^{-44} \mathrm{~cm}^{2}, c$ is the speed of light, $m_{e}$ is the electron mass. $\Phi$ is the angle between the neutrino and antineutrino beams, entering the annihilation rate formulation through the term $(1-\cos \Phi)$ (squared or not), which thus gives a stronger weighting to larger-angle collisions. This is what makes the dumbell-like morphology of BNS mergers such a prime candidate for $v_{i} \bar{v}_{i}$ annihilation over spherical configurations (see also the Appendix). $\langle\epsilon\rangle_{\nu_{i}}$ and $\langle\epsilon\rangle_{\bar{v}_{i}}$ are the $v_{i}$ and $\bar{v}_{i}$ mean neutrino energies, respectively, whose values we adopt for consistency from the simulations of Ruffert et al. (1997), i.e., 12,20 , and $27 \mathrm{MeV}$ for $v_{e}, \bar{v}_{\mathrm{e}}$, and " $v_{\mu}$ ", respectively (our values are within a few times $10 \%$ of these, so this has little impact on our discussion). Note also that the average neutrino energies for all three species are much larger than $m_{e} c^{2}$, and therefore make the second term in the above equation negligible (it is about a factor of 1000 smaller than the first one, and also has a much weaker large-angle weighting). The total annihilation rate is then the sum $Q^{+}\left(v_{e} \bar{v}_{\mathrm{e}}\right)+Q^{+}\left(" v_{\mu} \bar{v}_{\mu}\right.$ ").

In practice, we turn the angle integrals into discretized sums through the transformation

$$
\oint_{4 \pi} d \Omega I_{v} \longrightarrow \sum_{k} \Delta \Omega_{k} \cdot I_{\nu, k}
$$

where $\Delta \Omega_{k}$ is the solid angle subtended by the cell $k$ as seen from the location $\vec{r}$. Ruffert et al. (1997) applied their method to three-dimensional Cartesian simulations, while our simulations 
are two-dimensional and have both axial symmetry, and mirror symmetry about the equatorial plane. Making use of these symmetry properties, we remap our VULCAN/2D simulation from a two-dimensional (cylindrical) meridional slice onto a three-dimensional spherical volume which covers the space with a uniform grid of 40 zones for the $2 \pi$ azimuthal direction and 20 zones for the $\pi$ polar direction, while the reduced radial grid has a constant spacing in the log and uses 20 zones between 12 and $120 \mathrm{~km}$. We compute the annihilation rate at locations in a two-dimensional meridional slice of this new volume, with 16 uniformly spaced angles from the pole to the equator, and 40 zones with a constant spacing in the log between 15 and $120 \mathrm{~km} .{ }^{10}$ To estimate the annihilation rate at $\vec{r}$, one needs to estimate the flux received from all cells $\vec{r}_{k}$. Ruffert et al. (1997) assume that neutrino radiation is isotropic in the half-space around the outward direction given by the density gradient $\vec{n}_{\rho}$ at $\vec{k}$. Moreover, they approximate each emitting cell volume as a sphere, whose radius at $\left(r_{k}, \theta_{k}, \phi_{k}\right)$ is

$$
D_{k}=\left(\frac{\Delta \phi_{k} \Delta \mu_{k} \Delta r_{k}^{3}}{4 \pi}\right)^{1 / 3}
$$

where $\mu_{k}=\cos \theta_{k}$. With our location dependent cell volume, we obtain

$$
\sum_{k} \Delta \Omega_{k} \cdot I_{\nu, k} \approx \frac{1}{\pi} \sum_{k} \frac{\Delta \mu_{k} \Delta \phi_{k} \Delta r_{k}^{3}}{3 d_{k}^{2}} Q^{\mathrm{eff}}\left(v_{i}\right),
$$

where $d_{k}=\left|\vec{r}-\vec{r}_{k}\right|$. When we sum over discrete cells, the cosine of the angle between $\vec{k}$ and $\vec{k}^{\prime}$ is given by $\cos \Phi_{k k^{\prime}}=$ $\left(\vec{r}-\vec{r}_{k}\right)\left(\vec{r}-\vec{r}_{k^{\prime}}\right) /\left(\mid \vec{r}-\vec{r}_{k} \| \vec{r}-\vec{r}_{k^{\prime}}\right)$.

Following Ruffert et al. (1997), we apply selection criteria to determine whether to include a contribution. Specifically, emission and deposition sites must be in regions with a density lower than $10^{11} \mathrm{~g} \mathrm{~cm}^{-3}$. Interestingly, the leakage scheme predicts a very small "effective" emissivity from highdensity regions, due to the very long diffusion times from those optically thick regions. Relaxing the density cuts in the calculations leads only to a $50 \%$ enhancement in volumeintegrated annihilation rate $\dot{E}\left(v_{e} \bar{v}_{\mathrm{e}}\right)$, but an increase of a factor of $\sim 20$ for $\dot{E}$ (“ $v_{\mu} \bar{v}_{\mu}$ "). As pointed out earlier, the location in very high-density regions of the " $v_{\mu}$ " emitting cells means that most of the emitting volume is truncated by the adopted $10^{11} \mathrm{~g} \mathrm{~cm}^{-3}$ density cut, while results converge when this cut is increased to densities of $\sim 10^{13} \mathrm{~g} \mathrm{~cm}^{-3}$. Even with the latter, $\dot{E}\left(\right.$ " $v_{\mu} \bar{v}_{\mu}$ ") is still three orders of magnitude smaller than $\dot{E}\left(v_{e} \bar{v}_{\mathrm{e}}\right)$, likely a result of the neglect of bremsstrahlung, and the unfavorable $\left(1-\cos \Phi_{k k^{\prime}}\right)^{2}$ for this compact emitting configuration.

By contrast with the rather large uncertainty introduced through the somewhat arbitrary density cuts, the annihilation rate calculation is weakly affected by increasing the resolution. In the no-spin BNS model at $50 \mathrm{~ms}$, changing the number of radial-angle zones for the deposition sites $\left\{n r_{\text {edep }}, n t_{\text {edep }}\right\}$ from $(40,16)$ to $(60,48)$ and for emitting sites $\left\{n r_{\text {emit }}, n t_{\text {emit }}, n p_{\text {emit }}\right\}$ from $(20,20,40)$ to $(30,30,60)$ increases $\dot{E}\left(v_{e} \bar{\nu}_{\mathrm{e}}\right)$ from $2.82 \times$ $10^{49}$ to $3.14 \times 10^{49} \mathrm{erg} \mathrm{s}^{-1}$ (a $\sim 10 \%$ increase) and increases $\dot{E}\left(\right.$ “ $v_{\mu} \bar{v}_{\mu}{ }_{\mu}$ ") from $6.23 \times 10^{44}$ to $7.59 \times 10^{44} \mathrm{erg} \mathrm{s}^{-1}(\mathrm{a} \sim 20 \%$ increase). The same test done for the no-spin BNS model at $60 \mathrm{~ms}$ after the start of the simulations yields a $\dot{E}\left(v_{e} \bar{v}_{\mathrm{e}}\right)$ which is

\footnotetext{
10 We also perform higher-resolution accuracy-check calculations, but our results are already converged at this lower resolution.
}

identical to within $1 \%$, while $\dot{E}$ ( “ $v_{\mu} \bar{v}_{\mu}$ ") increases by $\sim 10 \%$ in the higher-resolution model. Hence, higher resolution does not change these values by a significant amount.

In the left half of each panel in Figure 16, we show the distribution in a two-dimensional meridional slice (it is in fact axisymmetric by construction) for the annihilation rate $Q^{+}\left(v_{e} \bar{v}_{\mathrm{e}}\right)$ of electron-type neutrinos, for the BNS merger models with initially no spins (left), co-rotating spins (middle), and counter-rotating spins (right). Volume-integral values $E\left(\nu_{\mathrm{i}} \bar{v}_{\mathrm{i}}\right)$ as a function of time for all three models are shown in Figure 17 (left panel; dashed line joined by star symbols) and given in Table 1 . The deposition rate is maximum near the poles, where the large-angle collisions occur, and close to the peak emission sites (as shown in Figure 15), i.e., near the SMNS surface, with peak values on the order of $10^{29} \mathrm{erg} \mathrm{cm}^{-3} \mathrm{~s}^{-1}$ for all three models. In this formalism, $\dot{E}\left(\right.$ “ $v_{\mu} \bar{v}_{\mu}$ ") (the middle panel of Figure 17) is at least three orders of magnitude smaller than $\dot{E}\left(v_{e} \bar{v}_{\mathrm{e}}\right)$. The peak volume-integrated energy deposition reaches a few $10^{50} \mathrm{erg} \mathrm{s}^{-1}$, typically two orders of magnitude below that achieved by charge-current reactions, whose associated energy deposition drives the neutrino-driven wind (Section 3; the right panel of Figure 17).

\subsection{Formalism Based on Multiangle Transport and Moments of the Specific Intensity}

We now present a formalism for the computation of the $v_{i} \bar{v}_{i}$ annihilation rate that directly exploits the neutrino-transport solution computed with VULCAN/2D in our BNS mergers, rather than using evaluations based on the leakage scheme (Ruffert et al. 1996, 1997).

Our approach is to use the angle-dependent neutrino-specific intensity calculated for snapshots at $10 \mathrm{~ms}$ intervals for these BNS merger models using our multiangle, $S_{n}$, scheme (Livne et al. 2004; Ott et al. 2008; Section 2.2). In the Appendix, and for completeness, we present such annihilation-rate calculations, but in the context of the postbounce phase of a $20 M_{\odot}$ progenitor (Ott et al. 2008) and of the accretion-induced collapse (AIC) of a massive and fast-rotating white dwarf (Dessart et al. 2006b). The formalism presented here applies to all cases equivalently.

Following Burrows et al. (2006b) and Janka (1991), the expression for the local energ-deposition rate $Q^{+}\left(v_{i} \bar{v}_{i}\right)$ at a position $\vec{r}$ due to annihilation of $v_{i} \bar{v}_{i}$ pairs into electron-positron pairs is given to leading order by

$$
\begin{aligned}
Q^{+}\left(\nu_{i} \bar{v}_{i}\right)= & \frac{1}{6} \frac{\sigma_{0}\left(C_{A}^{2}+C_{V}^{2}\right)_{v_{i} \bar{\nu}_{i}}}{c\left(m_{e} c^{2}\right)^{2}} \int_{0}^{\infty} d \varepsilon_{v} \int_{0}^{\infty} d \varepsilon_{v}^{\prime}\left(\varepsilon_{v}+\varepsilon_{v}^{\prime}\right) \\
& \times \oint_{4 \pi} d \Omega \oint_{4 \pi} d \Omega^{\prime} I_{v_{i}} I_{\bar{v}_{i}}^{\prime}(1-\cos \Phi)^{2},
\end{aligned}
$$

where $I_{v_{i}} \equiv I_{v_{i}}\left(\varepsilon_{v}, \vec{r}, \vec{n}, t\right)$ is the $v_{i}$-neutrino-specific intensity at energy $\varepsilon_{v}$, location $\vec{r}$, along the direction $\vec{n}$, and at time $t$. The primes denote the antiparticle. The angle $\Phi$ is the angle between the directions $\vec{n}$ and $\vec{n}^{\prime}$ of the neutrino and antineutrino, i.e., $\cos \Phi=\vec{n} \cdot \vec{n}^{\prime}$.

The formulation we present applies equally to all neutrino species, as long as the values of the weak coupling constants $C_{A}$ and $C_{V}$ are appropriately set. We have $C_{V}=1 / 2+2 \sin ^{2} \theta_{\mathrm{W}}$ for the electron types, $C_{V}=-1 / 2+2 \sin ^{2} \theta_{\mathrm{W}}$ for the $v_{\mu}$ and $v_{\tau}$ types, and $C_{A}= \pm 1 / 2$, with $\sin ^{2} \theta_{\mathrm{W}}=0.23$. The other variables have their usual meanings. Note that this formulation neglects the phase-space blocking by the final-state electron-positron pair which is relevant only at high densities and negligible 

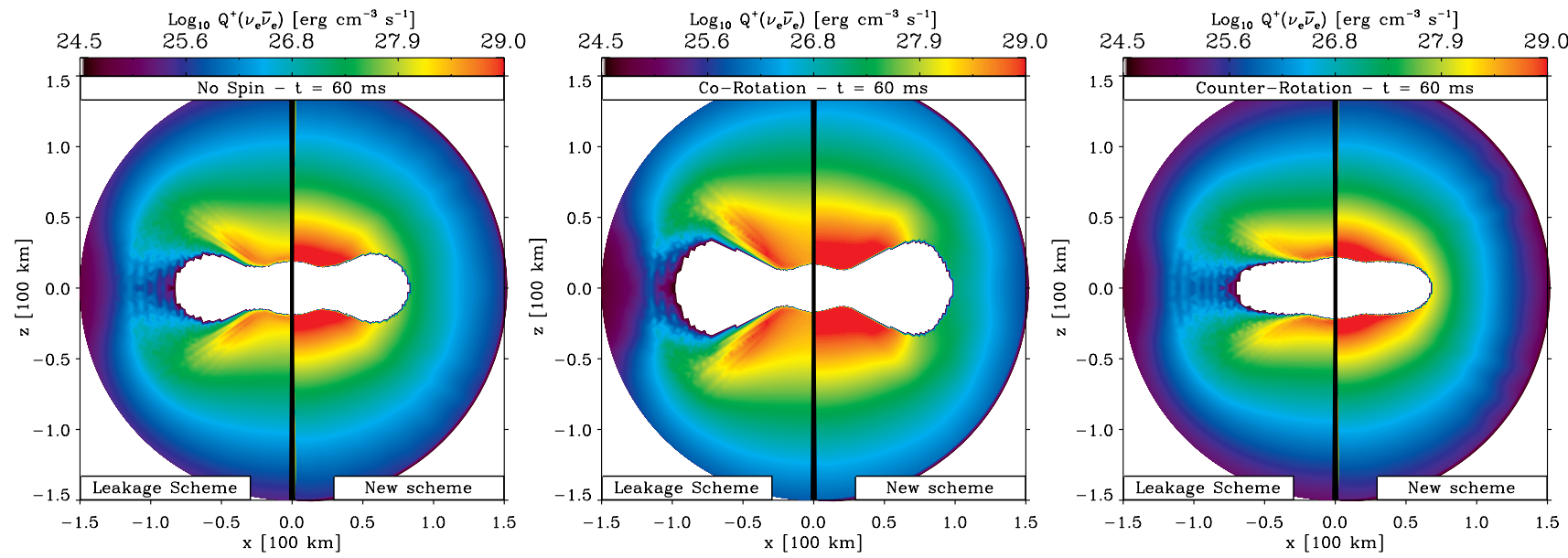

Figure 16. Colormaps of the log of the energy deposition $Q^{+}\left(v_{e} \bar{v}_{\mathrm{e}}\right)$ by electron-neutrino pair annihilation in the BNS merger models with initially no spin (left), co-rotating spins (middle), and counter-rotating spins (right), and at $60 \mathrm{~ms}$ after the start of the VULCAN/2D simulation. In each case, the evaluation is done with two different methods. In the left half of each panel, we show the results using the leakage scheme (Ruffert et al. 1996, 1997; see also Section 4.1). In the right half, we show the corresponding results using the new formalism presented in Section 4.2 using $16 \vartheta$-angles. We set the minimum of the color bar to white for all regions with densities greater than $10^{11} \mathrm{~g} \mathrm{~cm}^{-3}$. The maximum of the color bar is set at $10^{29} \mathrm{erg} \mathrm{cm}^{-3} \mathrm{~s}^{-1}$ to improve the visibility. Maximum values differ in fact by large factors between the two methods (in contrast with the good agreement in the cumulative values). For the model with no initial spins, we obtain maxima at $1.0 \times$ $10^{30}\left(S_{n}\right)$, compared with $6.5 \times 10^{28} \mathrm{erg} \mathrm{cm}^{-3} \mathrm{~s}^{-1}$ (leakage), and in the same order, we have $5.2 \times 10^{29}$ and $1.4 \times 10^{29} \mathrm{erg} \mathrm{cm}^{-3} \mathrm{~s}^{-1}$ for the co-rotating model, and $1.4 \times 10^{30}$ and $2.1 \times 10^{29} \mathrm{erg} \mathrm{cm} \mathrm{cm}^{-3} \mathrm{~s}^{-1}$ for the counter-rotating model. We do not show the distribution for the " $v_{\mu} \bar{v}_{\mu}$ " annihilation, which is qualitatively similar to that of $v_{e} \bar{v}_{\mathrm{e}}$, being merely weaker everywhere by about an order of magnitude. With the leakage scheme, the contrast in annihilation rate between these two neutrino types is even greater, primarily because " $v_{\mu}$ " neutrino emission occurs at densities in excess of $10^{12} \mathrm{~g} \mathrm{~cm}^{-3}$, thus, beyond our density cut. In the snapshots shown here and with the leakage scheme, we find integral net energy deposition rates by neutrino pair annihilation $\dot{E}\left(\nu_{\mathrm{i}} \bar{v}_{\mathrm{i}}\right)$ (summed over all neutrino species) of $1.78 \times 10^{49}, 4.43 \times 10^{49}$, and $1.50 \times 10^{49} \mathrm{erg} \mathrm{s}^{-1}$ for the BNS merger models with initially no spins, co-rotating spins, and counter-rotating spins, respectively. In the same order, but with the $S_{n}$ scheme (and using $16 \vartheta$-angles), we find $2.13 \times 10^{49}, 3.54 \times 10^{49}$, and $1.44 \times 10^{49} \mathrm{erg} \mathrm{s}^{-1}$. Note that these numbers are at least one to two orders of magnitude smaller than the corresponding rates due to charged-current neutrino absorption (see the left and right panels of Figure 17).

(A color version of this figure is available in the online journal.)
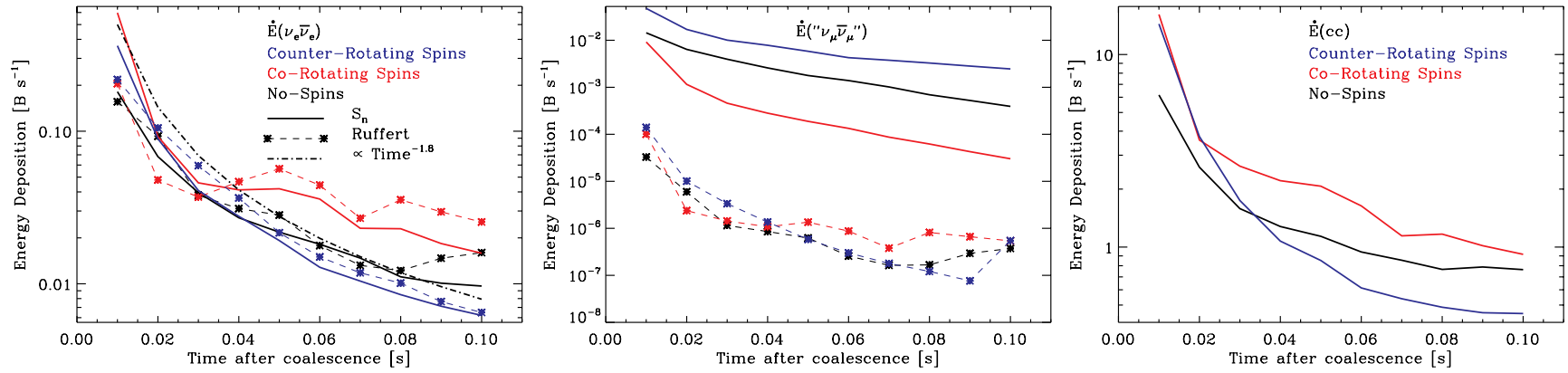

Figure 17. Volume-integrated energy deposition rate $\dot{E}\left(v_{e} \bar{v}_{\mathrm{e}}\right)$ (top) and the $\dot{E}\left(\right.$ “ $v_{\mu} \bar{v}_{\mu}$ ") (middle) neutrino-antineutrino annihilation processes, as well as that due to charge-current reactions (bottom) for the models initially with no spins (black), co-rotating spins (red), and counter-rotating spins (blue) and computed with the formalism presented in Section 4.2. The dashed lines (symbols highlight the times computed) represent for each model the corresponding result based on the leakage scheme (Section 4.1), following the method of Ruffert et al. $(1996,1997)$. The computations are performed every $10 \mathrm{~ms}$, from 10 to $100 \mathrm{~ms}$ after the start of the VULCAN/2D simulations. Note the fast decrease of the energy-deposition rate with time $\left(\propto t^{-1.8}\right.$; dotted line), following the decrease of all neutrino luminosities (see Figure 7) and the contraction of the SMNS. Note that Setiawan et al. $(2004,2006)$ obtain a slightly weaker dependence $\left(\propto t^{-1.5}\right)$, but with a black hole/torus-disk configuration and a treatment of the physical viscosity for energy dissipation through shear in the differentially rotating disk. Note that the energy unit used is the Bethe, i.e. $10^{51} \mathrm{erg} \equiv 1$ Bethe $[1 \mathrm{~B}]$.

(A color version of this figure is available in the online journal.)

in the semi-optically thin regime where pair annihilation may contribute to the net heating.

Now, setting the flux factor $\vec{h}=\vec{H} / J$ and the Eddingtontensor factor $\mathrm{k}=\mathrm{K} / J$, and by expanding the $\cos \Phi=\vec{n} \cdot \vec{n}^{\prime}$ term with an appropriate choice of the radiation unit vector (Hubeny \& Burrows 2007), we obtain for the general case in three dimensions

$$
\begin{aligned}
Q^{+}\left(v_{i} \bar{v}_{i}\right)= & \frac{8 \sigma_{0} \pi^{2}\left(C_{A}^{2}+C_{V}^{2}\right)_{v_{i} \bar{v}_{i}}}{3 c\left(m_{e} c^{2}\right)^{2}} \int_{0}^{\infty} d \varepsilon_{v} \int_{0}^{\infty} d \varepsilon_{v}^{\prime}\left(\varepsilon_{v}+\varepsilon_{v}^{\prime}\right) \\
& \times J_{v_{i}} J_{\bar{v}_{i}}^{\prime}\left(1-2 \vec{h}_{v_{i}} \cdot \vec{h}_{\bar{v}_{i}}^{\prime}+\operatorname{Tr}\left[\mathrm{k}_{v_{i}}: \mathrm{k}_{\bar{v}_{i}}^{\prime}\right]\right),
\end{aligned}
$$

where the term $\operatorname{Tr}\left[k_{v_{i}}: k_{\bar{v}_{j}}^{\prime}\right]$ is the trace of the matrix product of the two $J_{v}$-normalized Eddington tensors.
Assuming the special choice of cylindrical coordinates in axisymmetry and neglecting the velocity dependence of the radiation field, we obtain

$$
\begin{aligned}
Q^{+}\left(v_{i} \bar{v}_{i}\right)= & \frac{8 \sigma_{0} \pi^{2}\left(C_{A}^{2}+C_{V}^{2}\right)_{v_{i} \bar{v}_{i}}}{3 c\left(m_{e} c^{2}\right)^{2}} \int_{0}^{\infty} d \varepsilon_{v} \int_{0}^{\infty} d \varepsilon_{v}^{\prime}\left(\varepsilon_{v}+\varepsilon_{v}^{\prime}\right) \\
& \times J J^{\prime} \times\left(1-2 h_{r} h_{r}^{\prime}-2 h_{z} h_{z}^{\prime}\right. \\
& \left.+\mathrm{k}_{r r} \mathrm{k}_{r r}^{\prime}+\mathrm{k}_{z z} \mathrm{k}_{z z}^{\prime}+\mathrm{k}_{\phi \phi} \mathrm{k}_{\phi \phi}^{\prime}+2 \mathrm{k}_{r z} \mathrm{k}_{r z}^{\prime}\right)
\end{aligned}
$$

where we have suppressed the $v_{i}$ and $\bar{v}_{i}$ subscripts. Note that $k_{r z}$ is the only nonzero off-diagonal term in these coordinates. Using the trace condition on the Eddington tensor, $\mathrm{k}_{r r}+\mathrm{k}_{z z}+\mathrm{k}_{\phi \phi}=1$, 
the above can be recast into

$$
\begin{aligned}
Q^{+}\left(v_{i} \bar{v}_{i}\right)= & \frac{8 \sigma_{0} \pi^{2}\left(C_{A}^{2}+C_{V}^{2}\right)_{v_{i} \bar{v}_{i}}}{3 c\left(m_{e} c^{2}\right)^{2}} \int_{0}^{\infty} d \varepsilon_{v} \int_{0}^{\infty} d \varepsilon_{v}^{\prime}\left(\varepsilon_{v}+\varepsilon_{v}^{\prime}\right) \\
& \times J J^{\prime} \times\left(1-2 h_{r} h_{r}^{\prime}-2 h_{z} h_{z}^{\prime}+\mathrm{k}_{r r} \mathrm{k}_{r r}^{\prime}+\mathrm{k}_{z z} \mathrm{k}_{z z}^{\prime}\right. \\
& \left.+\left(1-\mathrm{k}_{r r}-\mathrm{k}_{z z}\right)\left(1-\mathrm{k}_{r r}^{\prime}-\mathrm{k}_{z z}^{\prime}\right)+2 \mathrm{k}_{r z} \mathrm{k}_{r z}^{\prime}\right) .
\end{aligned}
$$

For completeness and comparison with Janka (1991), we transform to spherical coordinates and reduce to spherically symmetric problems, which then yields

$$
\begin{aligned}
Q^{+}\left(v_{i} \bar{v}_{i}\right)= & \frac{8 \sigma_{0} \pi^{2}}{3 c\left(m_{e} c^{2}\right)^{2}}\left(C_{A}^{2}+C_{V}^{2}\right)_{v_{i} \bar{v}_{i}} \int_{0}^{\infty} d \varepsilon_{v} \int_{0}^{\infty} d \varepsilon_{v}^{\prime}\left(\varepsilon_{v}+\varepsilon_{v}^{\prime}\right) \\
& \times J J^{\prime} \times\left(1-2 h h^{\prime}+k k^{\prime}+\frac{1}{2}(1-k)\left(1-k^{\prime}\right)\right)
\end{aligned}
$$

with scalar $h$ and $k$. Equation (13) corresponds to the combination of Equations (1) and (6) of Janka (1991).

In practice, our approach is to start from snapshots of the (converged) MGFLD radiation-hydrodynamics simulations of the three BNS mergers presented in Section 3. Keeping the hydrodynamical variables frozen, the radiation variables are relaxed using the $S_{n}$ algorithm and with $8 \vartheta$-angles. As an accuracy check, we also compare the $S_{n}$ results based on simulations performed with $16 \vartheta$-angles. When we do this, the converged radiation field for the $8 \vartheta$-angle solution is remapped into the $16 \vartheta$-angle run (corresponding to a total of 144 angles), which is then relaxed. These simulations are quite costly, and take about 4 days with 48 processors. However, by contrast with the approach of Ruffert et al. (1996, 1997), the annihilation rates are then simply obtained from direct (local) integration of the moments of the neutrino-specific intensity.

In the right half of each panel in Figure 16, we show the distribution in a two-dimensional meridional slice for the annihilation rate $Q^{+}\left(v_{e} \bar{v}_{\mathrm{e}}\right)$ of electron-type neutrinos, for the BNS merger models with initially no spins (left), co-rotating spins (middle), and counter-rotating spins (right), but now computed from the results of the $S_{n}$ calculation and the formalism presented above. We provide in Figure 17 volume-integral values as a function of time for all three models (the top and middle panels; solid lines) and given in Table 1. By contrast with the leakage scheme results, the deposition is more evenly spread around the SMNS, is maximum at depth (in the region where the density cut applies), and reaches peak values a factor of a few larger. We find that it is not only the large-angle collisions that favor the deposition, but also the dependence on $J^{2}$ (and its $1 / R^{4}$ radial dependence in optically thin regions) which considerably weights the regions close to the radiating SMNS, making the deposition large not only in the polar regions, but also all around the SMNS. After $40 \mathrm{~ms}$, and although the associated neutrino luminosities are quite comparable between the three models, the (somewhat unrealistic) co-rotating spin BNS model boasts the largest annihilation rate. This stems from the very extended high-density SMNS configuration, leading to larger neutrinosphere radii and extended regions favoring large-angle collisions. This contrasts with the fact that it is the least hot of all three configurations, and is a somewhat weaker emitter, suggesting that it is not just the neutrino luminosity, but also the spatial configuration of the SMNS that sets the magnitude of the annihilation rate. In the new formalism, which employs results from the $S_{n}$ simulations, $\dot{E}$ (" $v_{\mu} \bar{v}_{\mu}$ ") (the middle panel of Figure 17) is now only one order of magnitude smaller than $\dot{E}\left(v_{e} \bar{v}_{\mathrm{e}}\right)$, even for the same adopted density cut. By contrast with the leakage scheme, and to some extent because of the treatment of bremsstrahlung processes, the emission from " $v_{\mu}$ " neutrinos is associated with more exterior regions of the SMNS, the $\sim 25 \mathrm{MeV}$ " $v_{\mu}$ " neutrinos decoupling at $\sim 18$ and $\sim 100 \mathrm{~km}$ along the polar and equatorial directions, respectively, and thus in regions with densities on the order of, or lower than, $10^{11} \mathrm{~g} \mathrm{~cm}^{-3}$ (Figure 11). The emission is, thus, not significantly truncated by the adopted density cut. Moreover, the subtended angle of the representative " $v_{\mu}$ " neutrinosphere is correspondingly much bigger, favoring larger-angle collisions.

This disagreement should not overshadow the good match between the leakage- and the $S_{n}$-scheme predictions for the $v_{e} \bar{v}_{\mathrm{e}}$ annihilation rate, which are typically within a few tens of percent, only sometimes in disagreement by a factor of $\sim 3$ (see, e.g., the no-spin BNS model at $10 \mathrm{~ms}$ ). The $S_{n}$-scheme results, thus, support the long-term decline of the annihilation rate predicted with the leakage scheme, but with a $\dot{E}\left(\right.$ “ $v_{\mu} \bar{v}_{\mu}$ ") value that is typically a factor of 10 smaller than $\dot{E}\left(v_{e} \bar{\nu}_{\mathrm{e}}\right)$ at all times. The results given here using $8 \vartheta$-angles are already fairly converged. For the co-rotating spin BNS model at $60 \mathrm{~ms}$ after the start of the simulation, we obtain the following differences between the 16 and $8 \vartheta$-angle $S_{n}$ calculations: $\dot{E}\left(v_{e} \bar{\nu}_{\mathrm{e}}\right)$ changes to $3.54 \times 10^{49}$ from $3.59 \times 10^{49} \mathrm{erg} \mathrm{s}^{-1}$ (down by $2 \%$ ), $\dot{E}$ (" $v_{\mu} \bar{v}_{\mu}$ ") changes to $1.73 \times 10^{47}$ from $1.34 \times 10^{47} \mathrm{erg} \mathrm{s}^{-1}$ (up by $29 \%$ ), and $\dot{E}(\mathrm{cc})$ changes to $1.40 \times 10^{51}$ from $1.64 \times 10^{51} \mathrm{erg} \mathrm{s}^{-1}$ (down by $15 \%$ ).

The long time-coverage of our simulations, up to $\gtrsim 100 \mathrm{~ms}$, shows that the peak value, which is also coincident with the peak neutrino luminosity at $5-10 \mathrm{~ms}$, is not sustained. The conditions at the peak of the annihilation rate do not correspond to a steady state, but instead herald the steady decrease that follows as the BNS merger radiates, contracts, and cools. In all simulations, $\dot{E}\left(v_{e} \bar{v}_{\mathrm{e}}\right)$ is down by a factor 10-100 at $100 \mathrm{~ms}$ compared to its peak value, while for $\dot{E}$ (" $v_{\mu} \bar{v}_{\mu}$ ") the decrease is by 2-3 orders of magnitude (this component is in any case subdominant). The $J^{2}$ dependence, combined with the strong fading of all neutrino emissivities, is at the origin of the steady decrease of the annihilation rate over the time span considered here. In their black hole/torus-disk configuration with $\alpha$-disk viscosity (which causes significant shear heating not accounted for here), Setiawan et al. (2004, 2006) observe that the annihilation rate decreases as $t^{-3 / 2}$, and, thus, only slightly more gradually than our prediction. In our work, neutrino energy deposition by charge-current reactions is the dominant means to counteract the global cooling effect of neutrino emission.

We now use the results for the $S_{n}$ scheme to discuss the assumption of Ruffert et al. $(1996,1997)$ that neutrino emission is quasi-isotropic. More precisely, they propose that the effective emission for every cell is isotropic in the half space around the outward direction given by the density gradient $\vec{n}_{\rho}$. By contrast, in the $S_{n}$ scheme, the angular distribution of the neutrino radiation field is solved for, and we can determine how isotropic this emission is. For that purpose, we study the spatial variation of the angular term in Equation (11),

$$
\begin{aligned}
W= & \left(1-2 h_{r} h_{r}^{\prime}-2 h_{z} h_{z}^{\prime}\right. \\
& \left.+k_{r r} k_{r r}^{\prime}+k_{z z} k_{z z}^{\prime}+k_{\varphi \varphi} k_{\varphi \varphi}^{\prime}+2 k_{r z} k_{r z}^{\prime}\right),
\end{aligned}
$$

and plot it in Figure 18 for the $v_{e}-\bar{v}_{e}$ annihilation rate at a representative neutrino energy of $12.02 \mathrm{MeV}$. Overplotting 


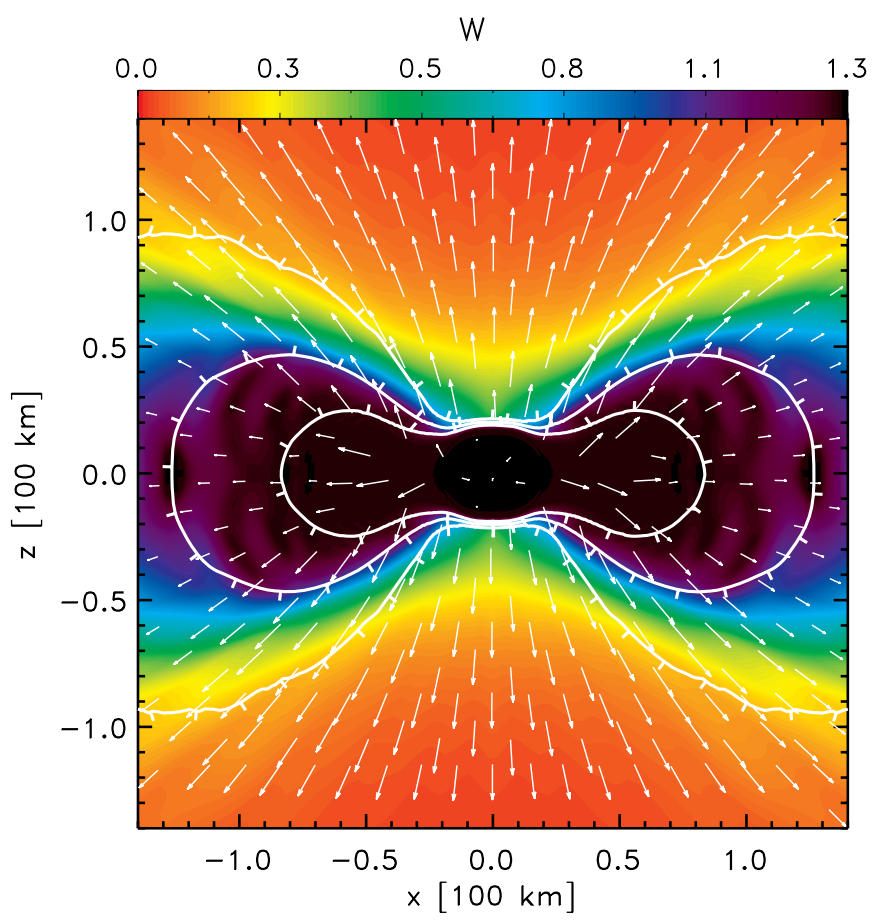

Figure 18. Angular factor $W$ in the $v_{i} \bar{v}_{i}$ annihilation rate (see Equations 11 and 14) shown for the $v_{e} \bar{v}_{e}$ process at $\varepsilon_{v}=12.02 \mathrm{MeV}$ for the BNS model with no initial spin. The snapshot corresponds to a time of $60 \mathrm{~ms}$ after the start of the VULCAN/2D simulation. We superpose $v_{e}$ flux vectors at the same $\varepsilon_{v}$ and density contours corresponding to $10^{9}, 10^{10}$, and $10^{11} \mathrm{~g} \mathrm{~cm}^{-3}$, indicating the local density gradient with perpendicular tick marks.

(A color version of this figure is available in the online journal.)

the (representative) $12.02 \mathrm{MeV} v_{e}$ flux vectors, together with isodensity contours, one sees that the flux is everywhere oriented predominantly in the radial direction, and peaks along the polar direction. The outward direction given by the local density gradient (given by the perpendicular-to isodensity contours, shown here as short white tick marks) is collinear to the flux vector along near-polar latitudes, while at midlatitudes they are perpendicular to each other. Hence, the assumption by Ruffert et al. $(1996,1997)$ of isotropic neutrino emission in the half space around the outward direction given by the local density gradient is not accurate in this instance. Note that it will likely hold more suitably once the black hole forms and only the torus disk radiates.

In Figure 18, $W$ has a maximum of $\sim 1.33$ in the optically thick regions of the extended (equatorial) disk-like structure. There, the flux-factor terms and $k_{\varphi \varphi}^{\prime}+2 k_{r z} k_{r z}^{\prime}$ are nearly zero, while $k_{r r} k_{r r}^{\prime}+k_{z z} k_{z z}^{\prime}+k_{\varphi \varphi} k_{\varphi \varphi}^{\prime}=1 / 3$. In polar regions, the transition to free streaming happens at small radii and $W$ decreases from $\sim 1.33$ at $\lesssim 20 \mathrm{~km}$ to $\sim 0.3$ at $40 \mathrm{~km}$. Outside $\sim 60-100 \mathrm{~km}$, $W \approx 0$, since $h_{z z} h_{z z}^{\prime} \approx k_{z z} k_{z z}^{\prime} \approx 1$ and all other terms are small. Considering now the fact that the distribution of the mean intensity, $J$, irrespective of energy group and species, is oblate inside the rapidly spinning SMNS and transitions to a prolate shape outside (see Figure 10), we can explain the spatial distribution of the pair annihilation rate (as shown in Figure 16). The rate peaks inside the oblate SMNS, since $J J^{\prime}$ and $W$ are largest there. Along the polar axis, $W$ decreases rapidly with radius, while $J J^{\prime}$ becomes prolate and remains large out to large radii, compensating in part for the small $W$. In equatorial regions, on the other hand, $J J^{\prime}$ drops off more rapidly, but $W$ remains near 1.33 out to a radius of $\sim 150 \mathrm{~km}$, and only slowly transitions to zero as the equatorial radiation field gradually becomes forward-peaked. This systematic behavior sustains a significant $v_{i} \bar{v}_{i}$ annihilation rate for equatorial radii of up to $\sim 150 \mathrm{~km}$. Note, however, that net energy deposition by pair annihilation does not occur in the equatorial charged-current loss regions.

The findings described in the above paragraph indicate that the dominant contribution to $Q^{+}\left(v_{i} \bar{v}_{i}\right)$ is not coming from large-angle collisions of neutrinos emitted from the disk, but rather is due to collisions at all angles and close to the highdensity, high-temperature, SMNS surface. This is in distinction to the annihilation rate computed using the leakage scheme and the assumptions concerning the morphology of the neutrino radiation field made in Ruffert et al. (1997). However, once the SMNS transitions to a black hole, the inner contribution will vanish and the radiation will come exclusively from the cooling hot torus. In a future paper we will address the annihilation rates that we obtain with this BNS merger configuration.

In the future, while retaining the merits of the $S_{n}$ method, we will need to improve the computation of the annihilation rates by accounting for GR effects. The compact and massive configuration of BNS mergers, with $G M / R c^{2}$ on the order of $20 \%$ at $20 \mathrm{~km}$, suggests that the numbers we present could be modified with this improvement, although Birkl et al. (2007) suggest the magnitude of the effect is at most a few tens of percent.

\section{CONCLUSION}

We have presented MGFLD radiation-hydrodynamics simulations of BNS mergers, starting from azimuthal-averaged twodimensional slices based on the three-dimensional SPH simulations produced with the MAGMA code (Rosswog \& Price 2007), for neutron star components with initially no spins, corotating spins, and counter-rotating spins. The main virtues of this work are (1) the solution of the radiation transport problem for $v_{e}, \bar{v}_{e}$, and " $v_{\mu}$ " neutrinos for eight energy groups, coupled to the hydrodynamical evolution of such mergers over a typical timescale of $\gtrsim 100 \mathrm{~ms}$ after the two components come into contact, and (2) the first quantitative assessment of baryon pollution by the neutrino-driven wind produced by such SMNSs. Interestingly, our results on neutrino signatures from such merger events confirm the broad adequacy of previous work that avoided solving the radiation transport problem by designing a neutrino trapping, or leakage, scheme (Ruffert et al. 1996, 1997; Rosswog \& Liebendörfer 2003). The only noticeable discrepancy is the much stronger " $v_{\mu}$ " luminosities that we predict with VULCAN/2D, something that may stem in part from the neglect of nucleon-nucleon bremsstrahlung in the above leakage schemes.

At $10 \mathrm{~ms}$ intervals and for each BNS merger model, we select a sequence of VULCAN/2D snapshots computed with the MGFLD solver and post-process them with the multiangle $S_{n}$ solver, relaxing the radiation variables, but freezing the fluid variables. Based on a knowledge of the energy-dependent, species-dependent, and angle-dependent neutrino-specific intensity $I_{\nu}\left(\varepsilon_{\nu}, \vec{n}\right)$, we compute the neutrino-antineutrino annihilation rate using a new formalism that incorporates various moments of $I_{v}\left(\varepsilon_{v}, \vec{n}\right)$. We find that the total annihilation rate computed with the $S_{n}$ solution and our new formalism is larger, but at most by a factor of a few, compared to the results based on a combination of a leakage scheme (Ruffert et al. 1996) and paired-cell summation (Ruffert et al. 1997). With density cuts alone, the annihilation rates based on the $S_{n}$ solution increase by a factor of about 2 . In our simulations, we find that all 


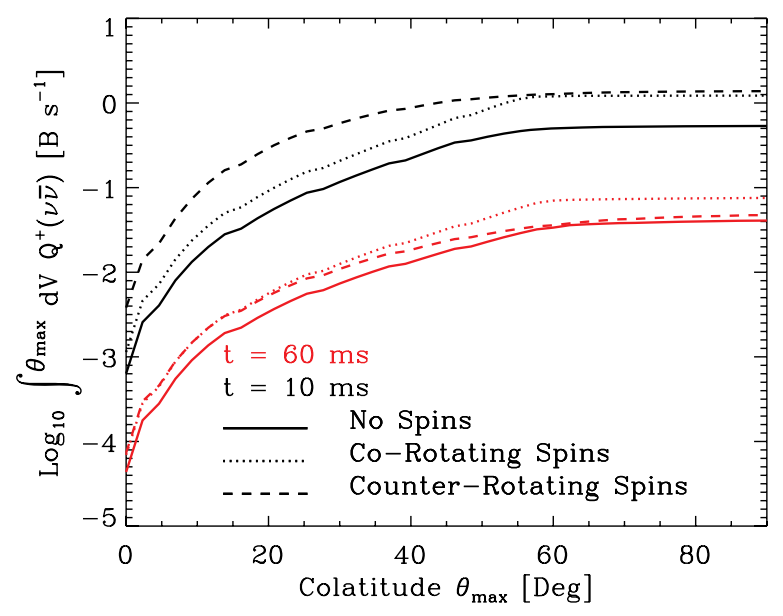

Figure 19. Variation of the $\log$ of the cumulative annihilation rate $\int^{\theta_{\max }} d V Q^{+}(\nu \bar{v})$ with polar angle $\theta_{\max }$ in the BNS merger models initially with no spin (solid), co-rotating spins (dotted), and counter-rotating spins (dashed), and shown here at a time of $10 \mathrm{~ms}$ (black) and $60 \mathrm{~ms}$ (red) after the start of the VULCAN/2D simulation. In other words, $\theta_{\max }$ is the half-opening angle of the cone that defines the integration volume for the quantity plotted along the ordinate axis. We use the $S_{n}$ results computed with $8 \vartheta$-angles and accounting only for a density cut for the sites of emission and deposition. Energy deposited in the cooling region is not subtracted off, yielding values larger by a factor of 2 compared with the corresponding values given in Table 1.

(A color version of this figure is available in the online journal.)

neutrino luminosities decrease after peak, resulting in a decrease in the annihilation rate with time, i.e., $\propto t^{-1.8}$. We find a cumulative total rate that decreases over $100 \mathrm{~ms}$ from a few $\times 10^{50}$ to $\sim 10^{49}$ erg $\mathrm{s}^{-1}$. Adopting an average energy of $8 k T \sim$ $40 \mathrm{MeV}$ for the annihilating $v_{e} \bar{v}_{e}$ (Burrows et al. 2006b), the number of $\mathrm{e}^{-} \mathrm{e}^{+}$pairs produced varies over that time span from a few $\times 10^{54}$ down to $\sim 10^{53} \mathrm{~s}^{-1}$.

We show in Figure 19 the cumulative annihilation rate computed with the $S_{n}$ solution and our new formalism at $10 \mathrm{~ms}$ (black) and $60 \mathrm{~ms}$ (red) after the start of the VULCAN/2D simulations. Only $\sim 1 \%$ of the total is deposited along the rotation axis and in a cone with a half-opening angle of $\sim 10^{\circ}$, yielding a rate of $\sim 10^{49} \mathrm{erg} \mathrm{s}^{-1}$ at $10 \mathrm{~ms}$ but down to $\sim 10^{48}$ erg s${ }^{-1}$ at $60 \mathrm{~ms}$. Integrating over a tenth of a second, we obtain a total energy deposition of $\lesssim 10^{48}$ erg. This result is about an order of magnitude smaller than obtained by Setiawan et al. (2004, 2006), although in their approach, neutrino emission is considerably boosted by shear heating in the differentially rotating disk. Their annihilation rates are stronger and weaken slightly more gradually with time, i.e., $\propto t^{-1.5}$. In the VULCAN/2D simulations presented here, we have no physical viscosity to include shear heating, nor is there adequate resolution to simulate the magneto-rotational instability. Our temperatures and neutrino emissions are therefore underestimates of what would occur in nature. With viscous dissipation as an energy source, we anticipate that our results might yield more attractive powers for the generation of a relativistic $\mathrm{e}^{-} \mathrm{e}^{+}-$ pair jet, an issue we defer to a future study.

Baryon loading of relativistic ejecta is a recognized problem in the production of a GRB, but this is the first time the neutrino-driven wind, the baryon polluter, has been simulated dynamically. All three BNS merger simulations we performed reveal the birth of a thermally driven wind through neutrino energy deposition in a gain layer at the surface, and primarily at high latitudes. The wind blows mostly along the polar funnel, contained in a cone with an opening angle of $\sim 20^{\circ}$ within $\mathrm{x} \lesssim 500 \mathrm{~km}$, but widening at larger distances to $\sim 90^{\circ}$. The electron fraction of the associated ejecta is close to 0.5 , along the pole, but is $\sim 0.1-0.2$ along all lower latitudes. Low $-Y_{\mathrm{e}}$ material at near-equatorial latitudes and large distances has a radial velocity below the local escape speed, and since local pressure gradients are not negligible, it is unclear whether it will eventually escape. Overall, it appears that if this preblack hole phase lasts for $100 \mathrm{~ms}, \lesssim 10^{-4} M_{\odot}$ of "r-process material" will feed the interstellar medium through this neutrinodriven wind. Moreover, such baryonic pollution along the polar direction may affect any subsequent relativistic ejecta, although the wind from the SMNS may last for only a short time, perhaps $100 \mathrm{~ms}$. Traveling at a tenth the speed of light, it will reach only out to 0.01 light second, thus much shorter than the duration of short GRBs. This initial wind phase cannot provide the sustained confinement needed for the potential relativistic ejecta. Although we focus on the phase prior to black hole formation, a neutrino-driven wind may blow after the black hole forms, too, but this time from the surrounding hot and dense torus, and driven by charge-current neutrino absorption at the disk surface. Importantly, because of the centrifugal barrier felt by particles coming in from sizable orbits in the disk, these injected baryons should remain away from the polar region, while neutrino-antineutrino annihilation would continue to contribute along the rotation axis of the SMNS, but now in an essentially baryon-free environment. This provides an attractive mechanism for confinement, since neutrino emission would yield both the power source for the relativistic ejecta (caused by neutrino-antineutrino annihilation) and the confined neutrino-driven wind (caused by charge-current reactions). In the future, we will investigate the neutrino signatures that are obtained in the context where the compact object is a black hole, and only the surrounding torus material radiates neutrinos. Such a disk wind would offer a natural confining ingredient for any relativistic ejecta propelled along the rotation axis and powered by annihilation of neutrinos and antineutrinos radiated from the disk, as proposed by Mochkovitch et al. (1993).

The huge amount of free energy of rotation, on the order of $10^{52} \mathrm{erg}$ in the SMNS and its torus disk, together with millisecond orbital periods, suggests that the magneto-rotational instability could play a major role in redistributing angular momentum, leading to solid-body rotation and increasing the magnetic pressure in the corresponding layers by orders of magnitude. Magneto-rotational effects should be strong and would lead to a considerably stronger neutrino-driven wind, as in the AIC of white dwarfs (Dessart et al. 2007), and delay the formation of a black hole. Similarly, Dessart et al. (2008) found that in collapsar candidates (Woosley \& Heger 2006; Yoon et al. 2006; Meynet \& Maeder 2007), the large amount of free energy of rotation in their iron core at the time of collapse can lead to a magnetically driven explosion, associated with very large mass loss rates which may compete with the mass accretion rate from the torus disk and jeopardize the formation of a black hole. By contrast with such collapsar models, SMNSs formed from merger events are unambiguously endowed with a large rotational-energy budget (stored in the orbital motion), of which a large fraction is differential and may be tapped. Hence, the phase prior to black hole formation should be modeled at very high resolution and in combination with neutrino transport to address these issues. The maximum neutron star mass allowed by the EOS will ultimately determine how much mass accretion can take place from the torus disk, and it thus represents a central question for short-duration GRBs. Presently, there is a lack of consensus that leaves this issue largely unsettled. 

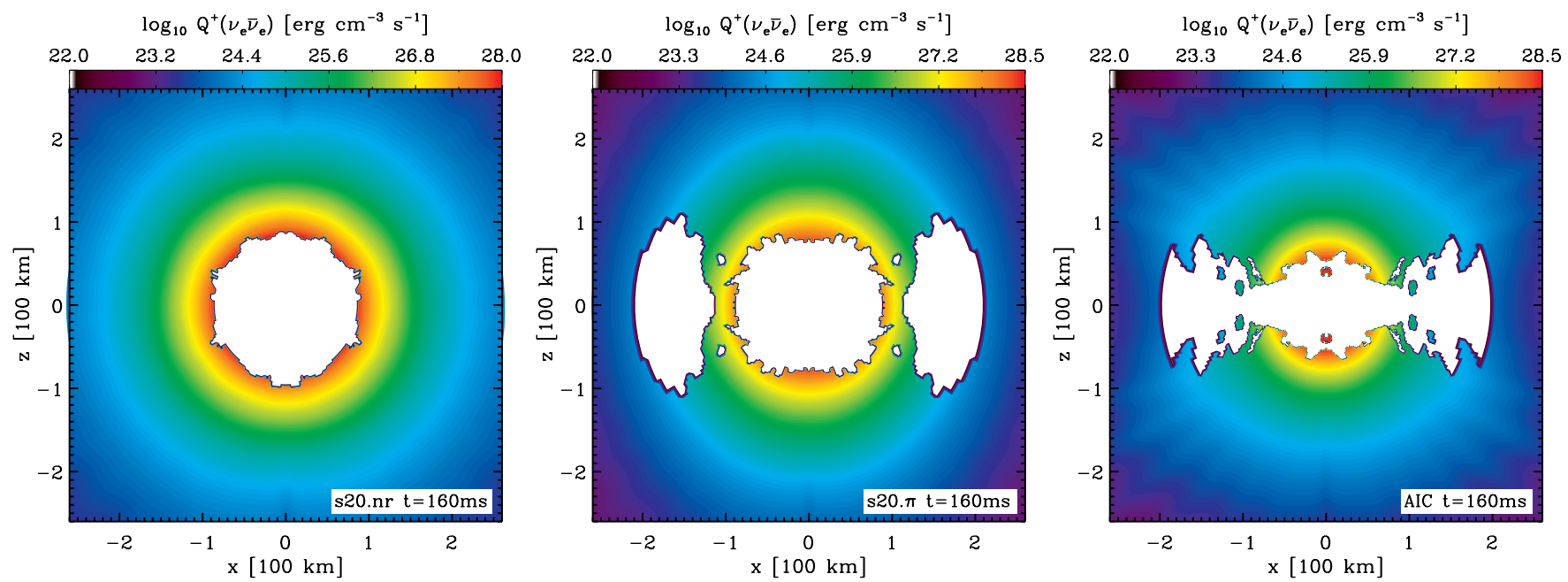

Figure 20. Colormaps of the energy deposition rate $Q^{+}\left(v_{e} \bar{\nu}_{\mathrm{e}}\right)$ by electron-neutrino pair annihilation in the nonrotating model s20.nr (left), the rotating model s20. $\pi$ (center), and in the 1.92- $M_{\odot}$ AIC model of Dessart et al. (2006b) (right), and in each case at $160 \mathrm{~ms}$ after bounce. The energy deposition by the " $v_{\mu} \bar{v}_{\mu}$ " annihilation process exhibits the same qualitative spatial distribution and is not shown here (the corresponding cumulative rates are typically a factor of 10 weaker than for $v_{e} \bar{v}_{e}$ ). The black lines demarcate regions in which charged-current losses dominate and regions deep inside the PNS where $\beta$-equilibrium prevails. Any energy deposition from pair annihilations is instantly reemitted in these regions by charged-current interactions and no net energy-deposition results. In the snapshots shown here, we find integral net energy-deposition rates by neutrino pair annihilation of $1.65 \times 10^{49}, 1.44 \times 10^{49}$, and $0.59 \times 10^{49} \mathrm{erg} \mathrm{s}^{-1}$ for models s20.nr, s20. $\pi$, and the AIC model, respectively. In this computation, we exclude the regions with a density larger than $10^{11} \mathrm{~g} \mathrm{~cm}^{-3}$, as well as regions of negative net gain (shown in white). These numbers are one to two orders of magnitude smaller than the corresponding rates due to charged-current neutrino absorption. Note that all models are run with $16 \vartheta$-angles.

(A color version of this figure is available in the online journal.)

Table 2

Cumulative $v_{i} \bar{v}_{i}$ Annihilation Rates

\begin{tabular}{|c|c|c|c|c|c|}
\hline Model Name & $\begin{array}{c}t-t_{b} \\
(\mathrm{~ms})\end{array}$ & $\begin{array}{c}\dot{E}(\mathrm{cc}) \\
\left(10^{49} \mathrm{erg} \mathrm{s}^{-1}\right) \\
\end{array}$ & $\begin{array}{c}\dot{E}\left(\nu_{e} \bar{v}_{e}\right) \\
\left(10^{49} \mathrm{erg} \mathrm{s}^{-1}\right)\end{array}$ & $\begin{array}{c}\dot{E}\left(“{ }^{\prime} v_{\mu} \bar{v}_{\mu} \text { ”) }\right. \\
\left(10^{49} \mathrm{erg} \mathrm{s}^{-1}\right)\end{array}$ & $\begin{array}{c}\text { Polar } \\
\text { Fall-Off }\end{array}$ \\
\hline s20.nr & 160 & 214.0 & 1.30 & 0.35 & $\propto \sim r^{-8.3}$ \\
\hline s20.nr & 500 & 81.8 & 0.77 & 0.12 & $\propto \sim r^{-9.5}-r^{-8.0}$ \\
\hline $\mathrm{s} 20 . \pi$ & 160 & 160.2 & 1.03 & 0.41 & $\propto \sim r^{-7.8}$ \\
\hline $\mathrm{s} 20 . \pi$ & 550 & 26.2 & 0.29 & 0.12 & $\propto \sim r^{-6.5}$ \\
\hline AIC $1.92-M_{\odot}$ & 160 & 53.7 & 0.42 & 0.17 & $\propto \sim r^{-8.3}$ \\
\hline $\mathrm{AIC} 1.92-M_{\odot}$ & 773 & 48.9 & 0.19 & 0.02 & $\propto \sim r^{-5.8}$ \\
\hline
\end{tabular}

Notes. Summary of the cumulative (but instantaneous) neutrino-antineutrino annihilation rate calculations. $t-t_{b}$ corresponds to the postbounce time of the computation. $\dot{E}(\mathrm{cc})$ is the integrated gain from charged-current interactions, while $\dot{E}\left(v_{e} \bar{v}_{e}\right)$ and $\dot{E}\left(\right.$ " $v_{\mu} \bar{v}_{\mu}$ ") denote the integral energy deposition by annihilation of $v_{e} \bar{v}_{e}$ and " $v_{\mu} \bar{\nu}_{\mu}$ " pairs (accounting for $v_{\mu} \bar{v}_{\mu}$ and $v_{\tau} \bar{v}_{\tau}$ ), respectively. The rightmost column gives the approximate power-law exponent that describes the radial fall-off of the energy deposition rates along the polar direction (we see the same distribution for both the $v_{e} \bar{\nu}_{e}$ and " $v_{\mu} \bar{v}_{\mu}$ " cases). For a spherically symmetric model in which neutrinos irradiate isotropically from a neutrinosphere, $Q^{+}\left(v_{i} \bar{v}_{i}\right) \propto r^{-8}$ at radii large compared to the neutrinosphere radius (Goodman et al. 1987). The ebbing annihilation rate with time results from its strong $J J^{\prime}$ dependence (Equation 11), and reflects PNS cooling (see the text for discussion.)

We acknowledge helpful discussions with and input from Ivan Hubeny, Casey Meakin, Jim Lattimer, Stan Woosley, H.Thomas Janka, Bernhard Müller, and Martin Obergaulinger. This work was partially supported by the Scientific Discovery through Advanced Computing (SciDAC) program of the US Department of Energy under grant numbers DE-FC0201ER41184 and DE-FC02-06ER41452. C.D.O. acknowledges support through a Joint Institute for Nuclear Astrophysics postdoctoral fellowship, sub-award 61-5292UA of NFS award 866004791. E.L. acknowledges support from ISF grant 805/04 The computations were performed at the local Arizona Beowulf cluster, on the Columbia SGI Altix machine at the Ames center of the NASA High-End Computing Program, at the National Center for Supercomputing Applications (NCSA) under Teragrid computer time grant TG-MCA02N014, at the Center for Computation and Technology at Louisiana State University, and at the National Energy Research Scientific Computing
Center (NERSC), which is supported by the Office of Science of the US Department of Energy under contract DE-AC0376 SF00098.

\section{APPENDIX}

In this appendix, we broaden the discussion on the $v_{i} \bar{v}_{i}$ annihilation process presented in Section 4, by moving away from BNS mergers, and focusing instead on 100-200 ms old PNSs, formed from the collapse of their $\mathrm{Fe}$ or $\mathrm{O} / \mathrm{Ne} / \mathrm{MG}$ core as it exceeded the Chandrasekhar mass. Such PNSs are hot because they are young, by contrast with BNS mergers, whose SMNSs are "rejuvenated" through shear heating and shocks associated with the coalescence phase. Specifically, we investigate the postbounce neutrino heating phase of the $20 M_{\odot}$ progenitor model of Woosley et al. (2002), adopting initially no rotation (model s20.nr) or differential rotation (model s20. $\pi$; the 
initial angular velocity at the center is $\pi \mathrm{rad} \mathrm{s}^{-1}$, equivalent to an initial central period of $2 \mathrm{~s}$ ). These models were evolved with the MGFLD and the $S_{n}$ solver using the radiation-hydrodynamics code VULCAN/2D, and the results were described in detail in Ott et al. (2008). To investigate the effect of very fast rotation in a PNS context, we also investigate the postbounce neutrino heating phase of the $1.92 M_{\odot}$ AIC model of Dessart et al. (2006b). To make a meaningful comparison of the $v_{i} \bar{v}_{i}$ annihilation rate in all three models, we take their properties at $160 \mathrm{~ms}$ after bounce (at times when the neutrino luminosities are comparable), and, thus, it is the shape of the radiating PNS that differs between models (for completeness, we also include some quantities at the last computed times-see Table 2). In this respect, our choice of models extends from the quasi-spherical s20.nr model to the mildly oblate s20. $\pi$ model, and finally to the strongly oblate (with a disk-like extension) AIC model. In all three models, the $S_{n}$ neutrino radiation field was computed with $16 \vartheta$-angles. Including the $\varphi$ direction, a total number of 144 angles were treated, following the prescription described in Ott et al. (2008).

In Figure 20, we present two-dimensional colormaps of the spatial distribution of the energy deposition by $v_{e} \bar{v}_{e}$ annihilation in the three model snapshots considered here. We draw as white the regions of high density $\left(\rho \gtrsim 10^{11} \mathrm{~g} \mathrm{~cm}^{-3}\right)$ where equilibration via charged-current interactions is fast, as well as regions in which cooling by charged-current interactions dominates. Any energy input by neutrino pair annihilation in these regions is overwhelmed by cooling through chargedcurrent processes and does not contribute to the net neutrino gain. Note also that we do not show the distribution for the “ $v_{\mu} \bar{v}_{\mu}$," because it is qualitatively similar to that of $v_{e} \bar{v}_{e}$, being merely weaker, i.e., with a volume-integrated magnitude that is a factor of about 10 smaller.

In the nonrotating model s20.nr, the $Q^{+}\left(v_{e} \bar{v}_{e}\right)$ distribution is approximately spherically symmetric, tracing regions of high neutrino-energy density in the neutrino-diffuse postshock region, and peaking near the lower edge of the gain region around $\sim 95 \mathrm{~km}$. The $\nu_{e} \bar{\nu}_{e}$ process dominates over that for " $v_{\mu} \bar{\nu}_{\mu}$," because the benefit of the favorable " $v_{\mu}$ " neutrino luminosity is more than canceled by the $\sim 5$ times smaller " $v_{\mu} \bar{v}_{\mu}$ " cross sections and by the smaller decoupling radii for “ $v_{\mu}$ " neutrinos, yielding systematically smaller collision angles.

In the $160 \mathrm{~ms}$ postbounce snapshot of model s20.nr, $Q^{+}\left(v_{i} \bar{v}_{i}\right)$ falls off radially as $\sim r^{-8.3}$ (both for $v_{e} \bar{\nu}_{e}$ and the " $v_{\mu} \bar{v}_{\mu}$ " annihilations), which is slightly steeper than the theoretical estimate $r^{-8}$ for $r \gg R_{\nu}$ made by Goodman et al. (1987). We find and list in Table 2 volume-integrated energy deposition rates of $1.30 \times 10^{49}$ and $0.35 \times 10^{49} \mathrm{erg} \mathrm{s}^{-1}$ for $v_{e} \bar{v}_{e}$ and $" v_{\mu} \bar{v}_{\mu}$ " annihilation processes, respectively. These rates are more than two orders of magnitude smaller than those associated with the net gain from charged-current reactions, which amount to $2.14 \times$ $10^{51} \mathrm{erg} \mathrm{s}^{-1}$ at that time. At $500 \mathrm{~ms}$ after bounce in the s20.nr model, the energy deposition rates have declined to $0.77 \times 10^{49}$ and $0.12 \times 10^{49} \mathrm{erg} \mathrm{s}^{-1}$ for $v_{e} \bar{v}_{e}$ and $" v_{\mu} \bar{v}_{\mu}$ " annihilation, respectively. At this time, we measure a more rapid radial fall-off $\propto r^{-9.5}$ for radii $\lesssim 100 \mathrm{~km}$ and somewhat shallower decline $\propto r^{-8}$ for larger radii. These results echo the steep decrease with time of the annihilation rate found for the BNS mergers discussed in this paper, suggesting that neutrino-cooled compact objects are quickly weakening neutrino-annihilation power sources in the absence of energy sources such as shear heating in a differentially rotating disk (Setiawan et al. 2004, 2006).
Our results for model s20.nr support the conclusions of previous studies (Cooperstein et al. 1986, 1987; Janka 1991) that argued that $v_{i} \bar{v}_{i}$ annihilation contributes little to the neutrino heating in quasi-spherical postbounce supernova cores. GR effects (bending of neutrino geodesics and redshift), which yield larger annihilation rates for very compact configurations (Salmonson \& Wilson 1999; Bhattacharyya et al. 2007; Birkl et al. 2007), are most likely irrelevant at the large radii at which annihilation may have any significance in our models. $G M / R c^{2}$ is already as low as $\sim 0.02$ at the inner edge of the polar gain region in models s20.nr and s20. $\pi$ and not larger than $\sim 0.06$ in the AIC snapshot.

Model s20. $\pi$ is rapidly rotating and has large pole-equator neutrino flux asymmetries (Ott et al. 2008). Though prolate and quickly varying with radius, the $Q^{+}\left(v_{i} \bar{v}_{i}\right)$ distributions in model s $20 . \pi$ do not vary with angle by more than a factor of 2 (neglecting for now the large equatorial cooling regions between $\sim 100$ and $200 \mathrm{~km}$ ). Along the poles, charged-current losses negate net energy deposition by $v_{i} \bar{v}_{i}$ annihilation at radii $\lesssim 80 \mathrm{~km}$. Again, $Q^{+}\left(v_{e} \bar{v}_{e}\right)$ dominates over $Q^{+}\left(“ v_{\mu} \bar{v}_{\mu}\right.$ ”), while both decrease with radius by $\sim r^{-7.8}$ at $160 \mathrm{~ms}$ after bounce and $\sim r^{-6.5}$ at $550 \mathrm{~ms}$ after bounce. As listed in Table 2, we find at $160 \mathrm{~ms}$ after bounce volume-integrated energy depositions of $1.03 \times 10^{49}$ and $0.41 \times 10^{49} \mathrm{erg} \mathrm{s}^{-1}$, for $v_{e} \bar{v}_{e}$ and " $v_{\mu} \bar{v}_{\mu}$," respectively. At $550 \mathrm{~ms}$ after bounce, these values have decreased to $0.29 \times 10^{49}\left(v_{e} \bar{v}_{e}\right)$ and $0.12 \times 10^{49}$ $\operatorname{erg~s}^{-1}$ (" $v_{\mu} \bar{v}_{\mu}$ "). The total annihilation contribution to neutrino heating is equivalent to $\sim 1 \%(\sim 1.5 \%)$ at $160 \mathrm{~ms}(550 \mathrm{~ms})$.

The right panel in Figure 20 shows the energy deposition by $v_{e} \bar{v}_{e}$ annihilation in the AIC model at $160 \mathrm{~ms}$ after bounce (see, Dessart et al. 2006b for details). In this rapidly rotating oblate PNS (the central period is $\sim 2.2 \mathrm{~ms}$ at this postbounce time), neutrinos are emitted from both the central object and its extended, moderately hot equatorial disk-like structure (more specifically from the pole-facing side of these side lobes). Significant energy deposition by neutrino pairs occurs at small radii in a wide polar wedge of $\sim 60^{\circ}$ (widening with radius to $\left.\sim 120^{\circ}\right)$ and $Q^{+}\left(v_{e} \bar{\nu}_{e}\right)$ reaches peak rates near the PNS surface at $\sim 15 \mathrm{~km}$ of up to $10^{29} \mathrm{erg} \mathrm{cm}^{-3} \mathrm{~s}^{-1}$, with $Q^{+}\left(" v_{\mu} \bar{v}_{\mu}\right.$ ") being globally smaller by an order of magnitude. The radial decline of $Q^{+}\left(v_{i} \bar{\nu}_{i}\right)$ along the polar direction becomes shallower with time, with power-law exponents varying from $\sim-8.3$ to $\sim-5.8$ from 160 to $773 \mathrm{~ms}$ after bounce.

In this AIC model, both $Q^{+}\left(v_{e} \bar{\nu}_{e}\right)$ and $Q^{+}$(“ $v_{\mu} \bar{v}_{\mu}$ ") reach local values at small radii that are of the same order of magnitude as the peak values in energy deposition per unit volume by charged-current interactions, yet the very limited volume of the high- $Q^{+}\left(v_{i} \bar{v}_{i}\right)$ gain regions leads to only very modest integral values at $160 \mathrm{~ms}$ after bounce of $0.42 \times 10^{49}$ and $0.17 \times$ $10^{49} \mathrm{erg} \mathrm{s}^{-1}$ for $v_{e} \bar{v}_{e}$ and " $v_{\mu} \bar{\nu}_{\mu}$ " pair annihilation, respectively, in our Newtonian model. At $773 \mathrm{~ms}$ after bounce and in the same order, these values are $0.19 \times 10^{49}$ and $0.02 \times 10^{49} \mathrm{erg} \mathrm{s}^{-1}$, respectively. Compared with the charged-current interactions driving the post-explosion wind phase of the AIC (Dessart et al. 2006b), even a 10-times-larger integral $\dot{E}\left(v_{i} \bar{v}_{i}\right)$ (e.g., via GR effects) would still amount to only $\lesssim 10 \%$ of the total charged-current energy deposition rate in this model.

\section{REFERENCES}

Abramovici, A., et al. 1992, Science, 256, 325

Akiyama, S., Wheeler, J. C., Meier, D. L., \& Lichtenstadt, I. 2003, ApJ, 584, 954 
Aloy, M. A., Janka, H.-T., \& Müller, E. 2005, A\&A, 436, 273

Anderson, M., Hirschmann, E. W., Lehner, L., Liebling, S. L., Motl, P. M., Neilsen, D., Palenzuela, C., \& Tohline, J. E. 2008, Phys. Rev. Lett., 100, 191101

Anderson, M., Hirschmann, E. W., Lehner, L., Liebling, S. L., Motl, P. M., Neilsen, D., Palenzuela, C., \& Tohline, J. E. 2008b, Phys. Rev. D, 77, 024006

Ardeljan, N. V., Bisnovatyi-Kogan, G. S., \& Moiseenko, S. G. 2005, MNRAS, 359,333

Asano, K., \& Fukuyama, T. 2000, ApJ, 531, 949

Asano, K., \& Fukuyama, T. 2001, ApJ, 546, 1019

Ayal, S., Piran, T., Oechslin, R., Davies, M. B., \& Rosswog, S. 2001, ApJ, 550, 846

Baiotti, L., Giacomazzo, B., \& Rezzolla, L. 2008, PRD, 78, 084033

Balbus, S. A., \& Hawley, J. F. 1991a, ApJ, 376, 214

Balbus, S. A., \& Hawley, J. F. 1991b, ApJ, 376, 223

Baumgarte, T. W., Shapiro, S. L., \& Shibata, M. 2000, ApJ, 528, L29

Benz, W., Cameron, A. G. W., Press, W. H., \& Bowers, R. L. 1990, ApJ, 348, 647

Bhattacharyya, A., Ghosh, S. K., Mallick, R., \& Raha, S. 2007, arXiv:707.2475 [astro-ph]

Bildsten, L., \& Cutler, C. 1992, ApJ, 400, 175

Birkl, R., Aloy, M. A., Janka, H.-T., \& Müller, E. 2007, A\&A, 463, 51

Bisnovatyi-Kogan, G. S., Popov, I. P., \& Samokhin, A. A. 1976, Ap\&SS, 41, 287

Blandford, R. D., \& Znajek, R. L. 1977, MNRAS, 179, 433

Burrows, A., Dessart, L., Livne, E., Ott, C. D., \& Murphy, J. 2007a, ApJ, 664, 416

Burrows, A., Livne, E., Dessart, L., Ott, C. D., \& Murphy, J. 2006a, ApJ, 640, 878

Burrows, A., Livne, E., Dessart, L., Ott, C. D., \& Murphy, J. 2007b, ApJ, 655, 416

Burrows, A., Reddy, S., \& Thompson, T. A. 2006b, Nucl. Phys., A, 777, 356

Calder, A. C., \& Wang, E. Y. M. 2002, ApJ, 570, 303

Centrella, J. M., New, K. C. B., Lowe, L. L., \& Brown, J. D. 2001, ApJ, 550 , L193

Cooperstein, J., van den Horn, L. J., \& Baron, E. 1987, ApJ, 321, L129

Cooperstein, J., van den Horn, L. J., \& Baron, E. A. 1986, ApJ, 309, 653

Dai, Z. G., Wang, X. Y., Wu, X. F., \& Zhang, B. 2006, Science, 311, 1127

Davies, M. B., Benz, W., Piran, T., \& Thielemann, F. K. 1994, ApJ, 431, 742

Dessart, L., Burrows, A., Livne, E., \& Ott, C. D. 2006a, ApJ, 645, 534

Dessart, L., Burrows, A., Livne, E., \& Ott, C. D. 2007, ApJ, 669, 585

Dessart, L., Burrows, A., Livne, E., \& Ott, C. D. 2008, ApJ, 673, L43

Dessart, L., Burrows, A., Ott, C. D., Livne, E., Yoon, S.-Y., \& Langer, N. 2006b, ApJ, 644, 1063

Di Matteo, T., Perna, R., \& Narayan, R. 2002, ApJ, 579, 706

Duez, M. D., Liu, Y. T., Shapiro, S. L., Shibata, M., \& Stephens, B. C. 2006, Phys. Rev. D, 73, 104015

Duez, M. D., Liu, Y. T., Shapiro, S. L., \& Stephens, B. C. 2004, Phys. Rev. D, 69, 104030

Eichler, D., Livio, M., Piran, T., \& Schramm, D. N. 1989, Nature, 340, 126

Faber, J. A., Grandclément, P., \& Rasio, F. A. 2004, Phys. Rev. D, 69, 124036

Faber, J. A., \& Rasio, F. A. 2000, Phys. Rev. D, 62, 064012

Faber, J. A., \& Rasio, F. A. 2002, Phys. Rev. D, 65, 084042

Faber, J. A., Rasio, F. A., \& Manor, J. B. 2001, Phys. Rev. D, 63, 044012

Freiburghaus, C., Rosswog, S., \& Thielemann, F.-K. 1999, ApJ, 525, L121

Freire, P. C. C., Wolszczan, A., van den Berg, M., \& Hessels, J. W. T. 2008, ApJ, 679, 1433

Goodman, J. 1986, ApJ, 308, L47

Goodman, J., Dar, A., \& Nussinov, S. 1987, ApJ, 314, L7

Hachisu, I. 1986, ApJS, 61, 479

Hubeny, I., \& Burrows, A. 2007, ApJ, 659, 1458

Janka, H.-T. 1991, A\&A, 244, 378

Janka, H.-T., Aloy, M.-A., Mazzali, P. A., \& Pian, E. 2006, ApJ, 645, 1305

Kidder, L. E., Will, C. M., \& Wiseman, A. G. 1992, Class. Quantum Grav., 9 , L125

Kiuchi, K., \& Kotake, K. 2008, MNRAS, 385, 1327

Kluźniak, W., \& Ruderman, M. 1998, ApJ, 505, L113

Kneller, J. P., McLaughlin, G. C., \& Surman, R. A. 2006, J. Phys. G: Nucl. Phys., 32, 443

Kochanek, C. S. 1992, ApJ, 398, 234

Lattimer, J. M., \& Schramm, D. N. 1974, ApJ, 192, L145

Lattimer, J. M., \& Schramm, D. N. 1976, ApJ, 210, 549

Lattimer, J. M., \& Swesty, F. D. 1991, Nucl. Phys., 535, 331

LeBlanc, J. M., \& Wilson, J. R. 1970, ApJ, 161, 541

Lee, W. H., \& Ramirez-Ruiz, E. 2007, New J. Phys., 9, 17

Levinson, A., \& Eichler, D. 2000, Phys. Rev. Lett., 85, 236

Liu, Y. T., \& Lindblom, L. 2001, MNRAS, 324, 1063
Livne, E. 1993, ApJ, 412, 634

Livne, E., Burrows, A., Walder, R., Lichtenstadt, I., \& Thompson, T. A. 2004, ApJ, 609, 277

Metzger, B. D., Thompson, T. A., \& Quataert, E. 2007, ApJ, 659, 561

Meynet, G., \& Maeder, A. 2007, A\&A, 464, L11

Mihalas, D., \& Mihalas, B. 1984, Foundations of Radiation Hydrodynamics (Mineola, NY: Dover)

Miller, W. A., George, N. D., Kheyfets, A., \& McGhee, J. M. 2003, ApJ, 583, 833

Mochkovitch, R., Hernanz, M., Isern, J., \& Martin, X. 1993, Nature, 361, 236

Moiseenko, S. G., Bisnovatyi-Kogan, G. S., \& Ardeljan, N. V. 2006, MNRAS, 370,501

Monaghan, J. J. 1997, J. Comput. Phys, 136, 298

Monaghan, J. J. 2002, MNRAS, 335, 843

Morris, J. P., \& Monaghan, J. J. 1997, J. Comput. Phys., 136, 41

Morrison, I. A., Baumgarte, T. W., \& Shapiro, S. L. 2004, ApJ, 610, 941

Nakamura, T., \& Oohara, K. 1989, Prog. Theor. Phys., 82, 1066

Nakamura, T., \& Oohara, K. 1991, Prog. Theor. Phys., 86, 73

Nakar, E. 2007, Phys. Rep., 442, 166

Narayan, R., Paczynski, B., \& Piran, T. 1992, ApJ, 395, L83

Nice, D. J., Splaver, E. M., Stairs, I. H., Löhmer, O., Jessner, A., Kramer, M., \& Cordes, J. M. 2005, ApJ, 634, 1242

Obergaulinger, M., Aloy, M. A., \& Müller, E. 2006, A\&A, 450, 1107

Oechslin, R., \& Janka, H.-T. 2006, MNRAS, 368, 1489

Oechslin, R., \& Janka, H.-T. 2007, Phys. Rev. Lett., 99, 121102

Oechslin, R., Janka, H.-T., \& Marek, A. 2007, A\&A, 467, 395

Oechslin, R., Rosswog, S., \& Thielemann, F.-K. 2002, Phys. Rev. D, 65,103005

Oohara, K., \& Nakamura, T. 1989, Prog. Theor. Phys., 82, 535

Oohara, K., \& Nakamura, T. 1990, Prog. Theor. Phys., 83, 906

Oohara, K., \& Nakamura, T. 1992, Prog. Theor. Phys., 88, 307

Ostriker, J. P., \& Mark, J. W.-K. 1968, ApJ, 151, 1075

Ott, C. D., Burrows, A., Dessart, L., \& Livne, E. 2008, ApJ, 685, 1069

Ott, C. D., Dimmelmeier, H., Marek, A., Janka, H.-T., Hawke, I., Zink, B., \& Schnetter, E. 2007, Phys. Rev. Lett., 98, 261101

Ott, C. D., Ou, S., Tohline, J. E., \& Burrows, A. 2005, ApJ, 625, L119

Paczynski, B. 1986, ApJ, 308, L43

Pessah, M. E., Chan, C.-K., \& Psaltis, D. 2006, Phys. Rev. Lett., 97, 221103

Pessah, M. E., Chan, C.-K., \& Psaltis, D. 2007, ApJ, 668, L51

Piran, T. 2005, Rev. Mod. Phys., 76, 1143

Price, D. 2004, PhD thesis, Univ. Cambridge (arXiv:astro-ph/0507472)

Price, D. J., \& Monaghan, J. J. 2007, MNRAS, 374, 1347

Price, D. J., \& Rosswog, S. 2006, Science, 312, 719

Rampp, M., Mueller, E., \& Ruffert, M. 1998, A\&A, 332, 969

Rosswog, S. 2005, ApJ, 634, 1202

Rosswog, S. 2007, RevMexAA, 27, 57-79

Rosswog, S., \& Davies, M. B. 2002, MNRAS, 334, 481

Rosswog, S., Davies, M. B., Thielemann, F.-K., \& Piran, T. 2000, A\&A, 360, 171

Rosswog, S., \& Liebendörfer, M. 2003, MNRAS, 342, 673

Rosswog, S., Liebendörfer, M., Thielemann, F.-K., Davies, M. B., Benz, W., \& Piran, T. 1999, A\&A, 341, 499

Rosswog, S., \& Price, D. 2007, MNRAS, 379, 915

Rosswog, S., \& Price, D. J. 2008, arXiv e-prints, 802

Rosswog, S., \& Ramirez-Ruiz, E. 2003, MNRAS, 343, L36

Rosswog, S., Ramirez-Ruiz, E., \& Davies, M. B. 2003, MNRAS, 345, 1077

Rosswog, S., Ramirez-Ruiz, E., Hix, W. R., \& Dan, M. 2008, Comput. Phys. Commun., 179, 184

Ruffert, M., \& Janka, H.-T. 1999, A\&A, 344, 573

Ruffert, M., Janka, H.-T., \& Schaefer, G. 1996, A\&A, 311, 532

Ruffert, M., Janka, H.-T., Takahashi, K., \& Schaefer, G. 1997, A\&A, 319, 122

Saijo, M., Baumgarte, T. W., \& Shapiro, S. L. 2003, ApJ, 595, 352

Salmonson, J. D., \& Wilson, J. R. 1999, ApJ, 517, 859

Setiawan, S., Ruffert, M., \& Janka, H.-T. 2004, MNRAS, 352, 753

Setiawan, S., Ruffert, M., \& Janka, H.-T. 2006, A\&A, 458, 553

Shen, H., Toki, H., Oyamatsu, K., \& Sumiyoshi, K. 1998a, Nucl. Phys., 637, 435

Shen, H., Toki, H., Oyamatsu, K., \& Sumiyoshi, K. 1998b, Prog. Theor. Phys., 100, 1013

Shibata, M., Duez, M. D., Liu, Y. T., Shapiro, S. L., \& Stephens, B. C. 2006 Phys. Rev. Lett., 96, 031102

Shibata, M., Taniguchi, K., \& Uryū, K. 2003, Phys. Rev. D, 68, 084020

Shibata, M., Taniguchi, K., \& Uryū, K. 2005, Phys. Rev. D, 71, 084021

Shibata, M., \& Uryū, K. 2002, Prog. Theor. Phys., 107, 265

Shibata, M., \& Uryu, K. 2007, Class. Quantum Grav., 24, 125

Springel, V., \& Hernquist, L. 2002, MNRAS, 333, 649

Stern, D. 1970, Am. J. Phys., 38, 494 
Sumiyoshi, K., Yamada, S., \& Suzuki, H. 2007, ApJ, 667, 382

Surman, R., McLaughlin, G. C., Ruffert, M., Janka, H., \& Hix, W. R. 2008, ApJ, 679, L117

Symbalisty, E., \& Schramm, D. N. 1982, Astrophys. Lett., 22, 143

Taylor, J. H., \& Weisberg, J. M. 1982, ApJ, 253, 908

Thompson, T. A., Burrows, A., \& Horvath, J. E. 2000, Phys. Rev. C, 62,035802

Usov, V. V. 1992, Nature, 357, 472

Usov, V. V. 1994, MNRAS, 267, 1035

Walder, R., Burrows, A., Ott, C. D., Livne, E., Lichtenstadt, I., \& Jarrah, M. 2005, ApJ, 626, 317
Weisberg, J. M., \& Taylor, J. H. 2005, in ASP Conf. Ser. 328, Binary Radio Pulsars, ed. F. A. Rasio \& I. H. Stairs (San Francisco, CA: ASP), 25

Wex, N., \& Schaefer, G. 1993, Class. Quantum Grav., 10, 2729

Wilson, J. R., Mathews, G. J., \& Marronetti, P. 1996, Phys. Rev. D, 54, 1317

Woosley, S. E., \& Heger, A. 2006, ApJ, 637, 914

Woosley, S. E., Heger, A., \& Weaver, T. A. 2002, Rev. Mod. Phys., 74, 1015

Yoon, S.-C., \& Langer, N. 2005, A\&A, 435, 967

Yoon, S.-C., Langer, N., \& Norman, C. 2006, A\&A, 460, 199

Zhuge, X., Centrella, J. M., \& McMillan, S. L. W. 1994, Phys. Rev. D, 50, 6247

Zhuge, X., Centrella, J. M., \& McMillan, S. L. W. 1996, Phys. Rev. D, 54, 7261 